\title{
Binap-gold(I) vs Binap-silver trifluoroacetate complexes as catalysts in 1,3-dipolar cycloadditions of azomethine ylides
}

\author{
María Martín-Rodríguez, ${ }^{[a]}$ Carmen Nájera, ${ }^{[a]}$ José M. Sansano ${ }^{[a]}$, Abel de Cózar, ${ }^{[b]}$ and Fernando \\ P. Cossío.*[b]
}

Dedication ((optional))

\begin{abstract}
The 1,3-dipolar precursors. The results obtained in cycloaddition between azomethine these transformations improve the ylides and alkenes is efficiently analogous ones obtained in the same catalyzed by $\left[\left(S_{\mathrm{a}}\right) \text {-Binap-AuTFA }\right]_{2}$. reactions catalyzed by Binap-AgTFA. Maleimides, 1,2-bis(phenylsulfonyl)- In addition, computational studies have ethylene, chalcone and nitrostyrene also been carried out in order to were suitable dipolarophiles even using demonstrate both the high sterically hindered 1,3-dipole enantioselection exhibited by de chiral
\end{abstract}

gold(I) complex, and the NLE observed in this transformation.

Keywords: $\mathrm{Au} \cdot \mathrm{Ag} \bullet 1,3$-dipolar
cycloaddition $\bullet$ azomethine $\bullet$
asymmetric synthesis $\bullet$ NLE $\bullet$

\section{Introduction}

The application of soluble gold complexes as catalysts for organic transformations has been widely explored for the last ten years. ${ }^{[1]}$ Spite of the numerous published gold(III)-catalysed processes, the diverse range of transformations developed by gold(I) complexes have demonstrated particular interest as catalysts. Gold(I) complexes catalyze reactions under very mild conditions giving, wide functional group compatibility and high efficiency. They act as soft carbophilic Lewis acids towards C-C multiple bonds. This high carbophilicity translates to a relative low oxophilic character of the gold(I) cation. However, recent advances in synthetic catalytic organic transformations have demonstrated that the weak heteroatom-gold(I) interaction is appropriate for the reaction

[a] M. Martín-Rodríguez, Prof. Dr. C. Nájera, Dr. J. M. Sansano. Department: Química Orgánica e Instituto de Síntesis Orgánica Institution: Universidad de Alicante. Address: Apdo 99, 03080-Alicante, Spain Fax: (+) 34-965903549

E-mail: cnajera@ua.es

[b] Dr. A. De Cózar, Prof. Dr. F. P. Cossío Department: Química Orgánica I Institution: Facultad de Química, Universidad del País Vasco Address P. K. 1072, E-20018 San Sebastián, Spain E-mail: fp.cossio@ehu.es

Supporting information for this article is available on the WWW under http://www.chemeurj.org/ or from the author. succeed. ${ }^{[1]}$ Its size, together with the already mentioned chemoselectivity and functional group compatibility are crucial features for application in complex crowded molecular structures. There are many processes which are sensitive to steric hindrance, the 1,3-dipolar cycloaddition $(1,3-\mathrm{DC})^{[2]}$ being one of them. The enantioselective version of 1,3-DC of stabilized azomethine ylides ${ }^{[3]}$ employing substoichiometric amounts ${ }^{[4]}$ of catalysts has been studied extensively since $2002^{[5]}$. Silver(I $)^{[6]}$ and copper(I $)^{[7]}$ catalyzed 1,3-dipolar cycloadditions offer excellent complementary results in terms of endo/exo-diastereoselection and are the most frequently employed metals nowadays.

This work, focused on the activity and efficiency of Binapsilver(I) salts complexes, can be considered as a evolution from the first attempt for the enantioselective 1,3-DC of azomethine ylides with dimethyl maleate described in 2002 by Zhang et al. using 3 mol \% of $\left(S_{\mathrm{a}}\right)$-Binap and $\mathrm{AgOAc}$ and $\mathrm{Et}_{3} \mathrm{~N}$ as base with very poor results. ${ }^{[5]}$ Our research group has found out that the very stable and recoverable $\left(S_{\mathrm{a}}\right)$ - or $\left(R_{\mathrm{a}}\right)$-Binap- $\mathrm{AgClO}_{4}$ complexes are able to catalyze the enantioselective 1,3-DC of azomethine ylides with maleimides. ${ }^{[8]}$ Using a less coordinating anion such as $\mathrm{AgSbF}_{6}$, instead of $\mathrm{AgClO}_{4}$, it was possible to achieve better results for bulky substrates and even with 1,3-bis(phenylsulfonyl)ethylene as a dipolarophile. $^{[6 \mathrm{e}]}$

Toste et al. published the first enantioselective 1,3-dipolar cycloaddition between mesoionic azomethine ylides (münchnones) with electron-poor alkenes catalyzed by $\mathrm{Cy}$-Segphos $(\mathrm{AuCl})_{2}$. The reaction afforded in all cases only the exo-adduct in high yields and very good enantioselections. ${ }^{[9]}$ However, the classical 1,3-dipolar cycloaddition of iminoesters and electrophilic olefins was not described using gold complexes. In this article, we present the general scope of this transformation using Binap-gold(I) complexes 
and a comparison with the analogous reactions performed with chiral Binap-silver(I) salts. ${ }^{[10][11}$

\section{Results and Discussion}

The gold(I) cation has only two coordination sites and its linear geometry makes asymmetric catalysis extremely difficult. Fortunately, a key to the development of enantioselecive gold(I)catalyzed transformations has been the identification of enantiomerically pure bis(gold)-chiral diphosphine complexes of the form $\left[(\mathrm{AuX})_{2}(\mathrm{P}-\mathrm{P})^{*}\right]$ as catalysts for enantioselective transformations. ${ }^{[1]} \mathrm{A}$ clear and recent example of the isolation, identification, and characterization of two chiral Binap-gold(I) complexes $\mathbf{1}$ and 2, bearing trifluoroacetate as counteranion, have been reported by Puddephatt et al. ${ }^{[12]}$ These complexes were prepared by mixing $\left(\mathrm{Me}_{2} \mathrm{~S}\right) \mathrm{AuCl}$ and the corresponding amount of the chiral Binap ligand. The resulting gold(I) chloride complexes were treated with different silver salts for $1 \mathrm{~h}$ in toluene and the suspension was filtered through a celite plug. The remaining solution was evaporated obtaining $\mathbf{1}$ or $\mathbf{2}$ in 89 and $96 \%$ yields, respectively. These cationic complexes were immediately employed without any other purification in the catalytic enantioselective 1,3DC of the imino ester 3a and $N$-methylmaleimide 4 (NMM) in toluene at $\mathrm{rt}$ (Scheme 1 and Table 1).
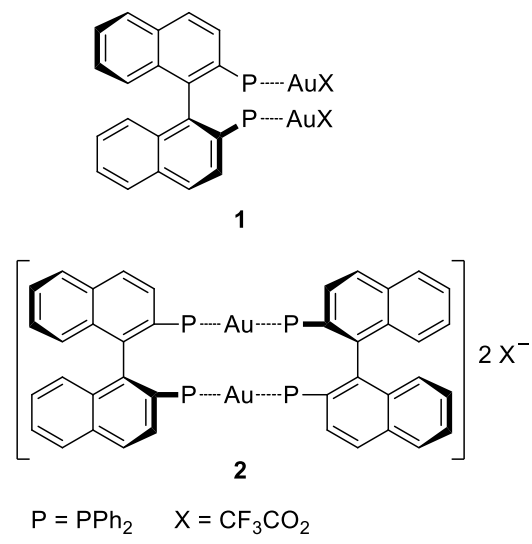

According to our experience, the model reaction between the imino ester (1,3-dipole precursor) 3a and $N$-methylmaleimide (NMM) 4a, in toluene at rt (Scheme 1 and Table 1), was selected for the initial optimization of the gold(I) catalytic efficiency. When this cycloaddition was performed in the presence of $10 \mathrm{~mol} \%$ of diisopropylethylamine (DIPEA) and $10 \mathrm{~mol} \%$ of complexes $(\text { BinapAuX })_{2} \mathbf{1}(\mathrm{X}=\mathrm{Cl}$ or TFA), product endo-5aa was obtained with high conversion but in racemic form (Table 1, entries 1 and 2). These two examples demonstrated that the chiral environment of the bisgold(I) complexes type $\mathbf{1}$ hardly participated in the enantiodiscriminating step. However, dimeric complexes type $(\text { BinapAuX })_{2} 2$ resulted to be the appropriate catalysts. Thus, in the case of the chiral complex $2(\mathrm{X}=\mathrm{OAc})$ product endo-5aa was obtained with high conversion and $60 \%$ ee in the presence of DIPEA (Table 1, entry 3). Interestingly, in the absence of base a $70 \%$ ee was obtained working the complex as a bifunctional catalyst (Table 1, entries 3 and 4). Better results were achieved when using complex $2(\mathrm{X}=\mathrm{OBz})$ in the presence of DIEA or in the absence of added base affording cycloadduct endo-5aa in $74 \%$ and $94 \%$ ee, respectively (Table 1 , entries 5 and 6). Although product endo-5aa was obtained in higher $94 \%$ ee in the absence of DIPEA, it was contaminated with secondary byproducts. The same inconvenience was detected when triethylamine was used as base in this cycloaddition reaction. When the gold(I) trifluoroacetate complex 2 $(\mathrm{X}=\mathrm{TFA}$ ) was used as catalyst, $74 \%$ ee of compound endo-5aa was obtained in the presence of DIPEA (Table 1, entry 7), whereas without base, $99 \%$ ee was obtained (Table 1, entry 8 ). This last reaction was performed introducing a previously synthesized (isolated and recrystallized) complex $2(\mathrm{X}=\mathrm{TFA})^{[12]}$ obtaining identical results (Table 1, entry 9). The enantiomeric form of cycloadduct endo-5aa was generated in $99 \%$ ee by the employment of the corresponding $(R, R)$-dimeric complex 2 ( $\mathrm{X}=\mathrm{TFA}$ ) (Table 1 , entry 10). Attempts to reduce the catalyst loading to a $5 \mathrm{~mol} \%$ werenot successful such as reveals the lower $60 \%$ ee obtained (Table 1, entry 11). At this point, an identical result was obtained independently of using the chiral complex 2 (X = TFA) (Table 1, entry 8$)$ and the chiral complex $\left(S_{\mathrm{a}}\right)$-Binap-AgTFA 6 in the absence of base (Table 1, entry 12). In every example the major cycloadduct obtained was the endo ( $>98: 2 d r)$ according to the NMR experiments of the crude products. Analogously, the absolute configuration was confirmed by comparison of their retention times using analytical chiral HPLC columns, and especific optical rotations with those described for enantiomerically pure samples.

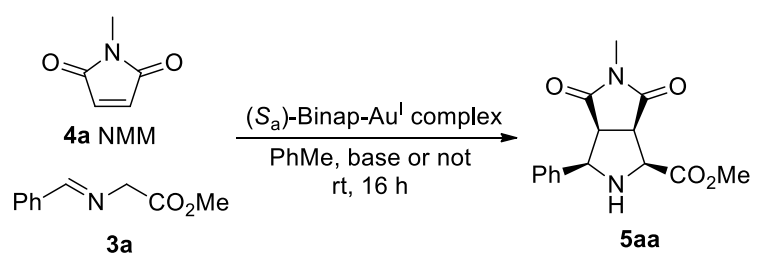

Scheme 1. Optimization of the 1,3-dipolar cycloaddition of $\mathbf{3 a}$ and $\mathbf{4 a}$.

Table 1. Optimization of the 1,3-dipolar cycloaddition of $\mathbf{3 a}$ and $\mathbf{4 a}$.

\begin{tabular}{|c|c|c|c|c|}
\hline Entry & Cat. $(10 \mathrm{~mol} \%)^{[\mathrm{a}]}$ & Base (10 mol\%) & $\operatorname{Conv}(\%)^{[b]}$ & $e e(\%)^{[c]}$ \\
\hline 1 & 1-Cl & DIPEA & $>95$ & rac \\
\hline 2 & 1-TFA & DIPEA & $>95$ & rac \\
\hline 3 & 2-OAc & DIPEA & $>95$ & 62 \\
\hline 4 & 2-OAc & - & $>95$ & 70 \\
\hline 5 & 2-OBz & DIPEA & $>95$ & 74 \\
\hline 6 & 2-OBz & - & $>95$ & $94^{[\mathrm{d}]}$ \\
\hline 7 & 2-TFA & DIPEA & $>95$ & 74 \\
\hline 8 & 2-TFA & - & $>95$ & 99 \\
\hline 9 & $2-\mathrm{TFA}^{[\mathrm{e}]}$ & - & $>95$ & 99 \\
\hline 10 & $2-\mathrm{TFA}^{[\mathrm{f}]}$ & - & $>95$ & ent-99 \\
\hline 11 & $2-\mathrm{TFA}^{[\mathrm{g}]}$ & - & 90 & 60 \\
\hline 12 & $\left(S_{\mathrm{a}}\right)$-Binap-AgTFA 6 & - & $>95$ & 99 \\
\hline
\end{tabular}

[a] The generation of gold catalysts was achieved by mixing tantamount equivalents of gold(I) complex 1 and the corresponding silver(I) salt. [b] Determined by ${ }^{1} \mathrm{H}$ NMR of the crude sample obained by using the filtered solution of the gold complex after anionic interchange. The observed endo:exo ratio was always $>98: 2\left({ }^{1} \mathrm{H} \mathrm{NMR}\right)$. [c] Determined by using analytical chiral HPLC columns (Daicel, Chiralpak AS). [d] 
Notable amounts of unidentified side products were observed ( ${ }^{1} \mathrm{H}$ NMR). [e] The reaction was carried out with the chiral complex isolated by recrystallization. [f] The reaction was performed with $\left(R_{\mathrm{a}}\right)$-Binap as ligand. [g] Reaction performed with $5 \mathrm{~mol} \%$ of catalyst.

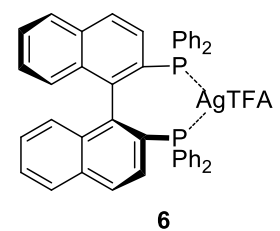

Comparative studies for this catalyzed enantioselective 1,3-DC were performed using gold(I) and silver(I) complexes together with different iminoesters and maleimides under the best reaction conditions depicted on Table 1 (Scheme 2 and Table 2). In the first example, the absence of base, (including the above described reaction of 3aa with NMM) performed with $N$-ethylmaleimide (NEM) $4 \mathbf{b}$ and $N$-phenylmaleimide (NPM) $4 \mathbf{c}$ products $5 \mathbf{a b}$ and $\mathbf{5 a c}$ were isolated with higher enantioselectivity when the reaction was catalyzed by the chiral gold complex (Table 2, entries 1, 3, and 5). However, in the presence of DIPEA (10 mol\%) the chiral silver(I) catalyzed process is more efficient except in the reaction done with NPM (Table 2, compare entries 1 with 2, 3 with 4 and 5 with 6). In fact, the result obtained when NPM was employed as a dipolarophile (Table 2, entry 5 ) is particularly noteworthy because it is the best $e e(80 \% e e)$ achieved till date with chiral Binap ligands. When $\left(S_{\mathrm{a}}\right)$-Binap-AgTFA 6 was used as catalyst, racemic product $\mathbf{5 a c}$ was obtained. For the 1,3-DC of other arylideneaminoesters $\mathbf{3 b}$ $\mathbf{h}$, and maleimides the conversions are identical independently of the metal cation employed. In the case of 2-naphthyl derived iminoester the enantioselections were very close to each other, but with NPM chiral gold(I) catalysts $2(X=$ TFA) (Table 2, entries 7-9) again was much more efficient generating the highest enantioselections. The dipole precursors $\mathbf{3 c}$, and $\mathbf{3 d}$, containing an ortho-substituent in the aryl moiety, were appropriate sterically hindered starters in the gold(I)-catalyzed 1,3-DC with NMM affording endo-compounds 5ca and 5da with 99 and $88 \%$ ee, respectively. In both examples the resulting enantioselections induced with the corresponding chiral silver(I) complex were very poor (Table 2 , entries 10 and 11). The para-substituted methyl iminoglycinates $\mathbf{3 e}, \mathbf{3 f}$, and $\mathbf{3 g}$ underwent the gold(I) and the silver(I)- mediated 1,3-DC obtaining no rational results in terms of enantioselection (Table 2, entries 12-14).

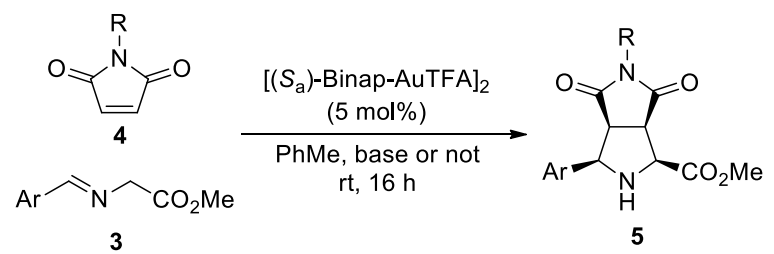

4a, R = Me (NMM); 4b, R = Et (NEM); 4c, R = Ph (NPM)

Scheme 2. $[(S) \text {-Binap-AuTFA }]_{2}$ Catalyzed 1,3-dipolar cycloaddition of iminoesters 3 and maleimides 4 .

The insertion of a substituent at the $\alpha$-position of the 1,3-dipole precursor was next evaluated. Thus, when methyl benzylidenephenylalaninate 7a was allowed to react with NMM under the standard reaction conditions, the reaction performed with the gold(I) complex needed $24 \mathrm{~h}$ more than the corresponding reaction using the analogous silver(I) complex for achieving almost total conversions (Scheme 3). The enantioselection showed by $\left[\left(S_{\mathrm{a}}\right)\right.$ Binap-AuTFA $]_{2}$ complex $(99 \%$ ee) was higher than in the case of using $\left(S_{\mathrm{a}}\right)$-BinapAgTFA $(65 \%$ ee $)$ as a catalyst. Product 8aa was obtained as endo-diastereoisomer ( $>98: 2$, determined by ${ }^{1} \mathrm{H}$ NMR spectroscopy).

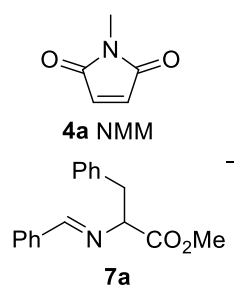

$7 a$

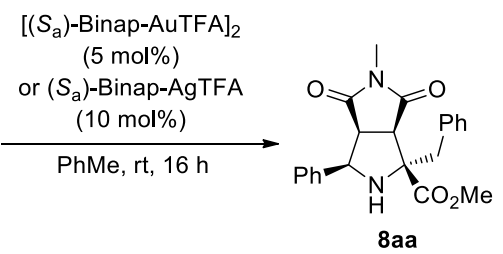

Au' complex: $3 d, 78 \%, 99 \%$ ee $\mathrm{Ag}^{\prime}$ complex: $2 \mathrm{~d}, 95 \%, 65 \%$ ee
Scheme 3. $[(S) \text {-Binap-AuTFA }]_{2}$ versus (S)-Binap-AgTFA Catalyzed 1,3-dipolar cycloaddition of iminoester $7 \mathbf{a}$ and NMM $4 \mathbf{a}$.

According to the published results, obtained from chiral Binapsilver(I) complexes, ${ }^{[8]}$ we also tested the efficiency of the Binapgold(I) trifluoroacetate complexes in the enantioselective cycloaddition of azomethine ylides and trans-1,2bis(phenylsulfonyl)ethylene 9, a synthetic equivalent of acetylene (Scheme 4 and Table 3). The reaction, performed with $5 \mathrm{~mol} \%$ of the gold(I) 2 (X = TFA) catalyst, afforded cycloadducts $\mathbf{1 0}$ in a non predictable enantioselectivities in the absence or in the presence of DIPEA $(10 \mathrm{~mol} \%)$ as base. In the case of product 10a, a lower enantiomeric excess was obtained when $\left(S_{\mathrm{a}}\right)$-Binap-AgTFA 6 was used as catalyst and better results were obtained in the absence of base (Table 3, compare entries 1 and 2). Compound endo-10b was obtained in better enantiomeric excesses in the absence of base (compare in Table 3, entries 3 and 4). The rest of the examples gave the best enantioselections in the absence of base (compare entries 5 and 6, 7 and 8,9 and 10). Again, the $p$-chloro substituted iminoester gave very poor ee (Table 3, entries 11 and 12).

The absolute configuration of the endo-cycloadducts was assigned according to the retention times using analytical chiral HPLC columns, and by comparison of the physical properties of the isolated samples with the properties published in the literature for the analogous compounds.

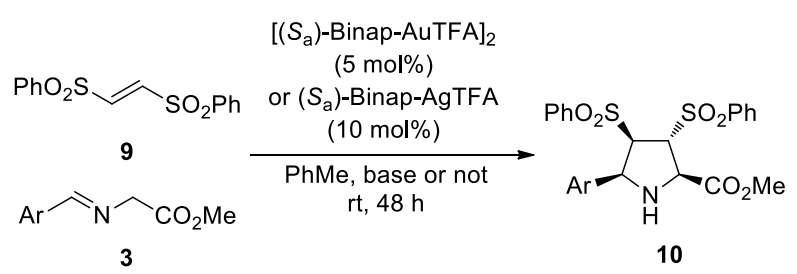

Scheme 4. $[(S) \text {-Binap-AuTFA }]_{2}$ versus (S)-Binap-AgTFA Catalyzed 1,3-dipolar cycloaddition of iminoesters 3 and disulfone $\mathbf{9}$.

Table 3. 1,3-DC of iminoesters 3a and disulfone 9 catalyzed by chiral complex $\mathbf{2}$ (X= TFA).

\begin{tabular}{|c|c|c|c|c|c|c|c|}
\hline & & & \\
\hline & & & & \multicolumn{2}{|c|}{ Gold catalysis } & \multicolumn{2}{|c|}{ Silver catalysis } \\
\hline $\begin{array}{l}\text { Ent } \\
\text { ry }\end{array}$ & Ar, 3 & $\begin{array}{l}\text { Base } \\
(10 \\
\text { mol\%) }\end{array}$ & 10 & $\begin{array}{l}\text { Yield } \\
(\%)^{[\mathrm{a}] \mathrm{b}} \\
]\end{array}$ & $\begin{array}{l}e e \\
(\%)^{[\mathrm{c}} \\
]\end{array}$ & $\begin{array}{l}\text { Yield } \\
(\%)^{[a][b]} \\
\text { b }\end{array}$ & $\begin{array}{l}e e \\
(\%)^{[\mathrm{c}][\mathrm{d}]}\end{array}$ \\
\hline 1 & $\mathrm{Ph}, \mathbf{3 a}$ & DIPEA & $10 \mathrm{a}$ & 81 & 80 & 80 & 86 \\
\hline 2 & $\mathrm{Ph}, \mathbf{3 a}$ & - & $10 \mathrm{a}$ & 74 & 99 & 81 & 96 \\
\hline
\end{tabular}




\begin{tabular}{|c|c|c|c|c|c|c|c|}
\hline 3 & $\begin{array}{l}\text { 2-Naphthyl, } \\
\text { 3b }\end{array}$ & DIPEA & $\begin{array}{l}10 \\
b\end{array}$ & 91 & 90 & 88 & 64 \\
\hline 4 & $\begin{array}{l}\text { 2-Naphthyl, } \\
\text { 3b }\end{array}$ & - & $\begin{array}{l}10 \\
\text { b }\end{array}$ & $<40$ & $\mathrm{rac}$ & $<40$ & $\mathrm{rac}$ \\
\hline 5 & $\begin{array}{l}2- \\
(\mathrm{MeO}) \mathrm{C}_{6} \mathrm{H}_{4}, \\
\text { 3i }\end{array}$ & DIPEA & $10 \mathrm{i}$ & 80 & 30 & 83 & 20 \\
\hline 6 & $\begin{array}{l}2- \\
(\mathrm{MeO}) \mathrm{C}_{6} \mathrm{H}_{4}, \\
\text { 3i }\end{array}$ & - & $10 \mathrm{i}$ & $<40$ & 40 & $<40$ & 20 \\
\hline 7 & 3-Pyridyl, 3e & DIPEA & $10 \mathrm{e}$ & 73 & 96 & 70 & 92 \\
\hline 8 & 3-Pyridyl, 3e & - & $10 \mathrm{e}$ & 73 & 96 & 70 & 96 \\
\hline 9 & $4-\mathrm{MeC}_{6} \mathrm{H}_{4}, \mathbf{3 f}$ & DIPEA & $10 f$ & 91 & 88 & 90 & 96 \\
\hline 10 & $4-\mathrm{MeC}_{6} \mathrm{H}_{4}, \mathbf{3 f}$ & - & $10 f$ & 67 & 99 & 75 & 96 \\
\hline 11 & $4-\mathrm{ClC}_{6} \mathrm{H}_{4}, \mathbf{3 h}$ & DIPEA & $\begin{array}{l}10 \\
h\end{array}$ & 81 & 22 & 80 & $\mathrm{rac}$ \\
\hline 12 & $4-\mathrm{ClC}_{6} \mathrm{H}_{4}, \mathbf{3 h}$ & - & $\begin{array}{l}10 \\
h\end{array}$ & $<40$ & $\mathrm{rac}$ & $<40$ & $\mathrm{rac}$ \\
\hline
\end{tabular}

[a] Isolated yields after flash chromatography (silica gel). [b] The observed endo:exo ratio was always $>98: 2$ ( ${ }^{1} \mathrm{H}$ NMR). [c] Determined by using analytical chiral HPLC columns. [d] Reaction performed with $\left(S_{\mathrm{a}}\right)$-Binap-AgTFA 6.

Searching for another appropriate dipolarophiles for this enantioselective catalyzed 1,3-DC of azomethine ylides, chalcone and $\beta$-nitrostyrene were used. As well as occurred in the 1,3-DC catalyzed by chiral phosphoramidites and silver perchlorate, chalcones reacted efficently affording exclusively very clean endocycloadducts $\mathbf{1 4}$ after $24 \mathrm{~h}$, at room temperature and in the presence of $\mathrm{Et}_{3} \mathrm{~N}(10 \mathrm{ml} \%)$ as base, rather than DIPEA, independently of the selected metal. The most important difference consisted of the enantioselection achieved. Whilst chiral dimeric gold catalyst furnished very high ee of $\mathbf{1 5}$, silver complex gave always lower enantiodiscriminations (Scheme 5a, and Table 4, entries 1-3). The reaction between iminoester $\mathbf{3 a}$ and $\beta$-nitrostyrene afforded very complex mixtures of unidentified compounds when chiral silver complexes were employed. However, very clean crude reaction products were obtained when dimeric $[(S) \text {-Binap-AuTFA }]_{2}$ was used. The diastereoselectivity was not so high than in precedent cycloadditions, obtaining in these examples 20:80 endo:exo mixtures of $16 \mathrm{a}$, spite of runnig the reaction at $-20^{\circ} \mathrm{C}$. Cycloadduct exo-16a was obtained in $70 \%$ or $60 \%$ ee whether the reaction was performed at $25{ }^{\circ} \mathrm{C}$ or $-20{ }^{\circ} \mathrm{C}$, respectively (Scheme $5 \mathrm{~b}$, and Table 4 , entries 4 and 5). The lowering of the temperature in the reactions involving chalcone or $\beta$-nitrostyrene afforded incomplete crude reaction mixtures after $48 \mathrm{~h}$, furnishing a lower enantioselectivity in products 15 or $16 \mathbf{a}$, respectively.

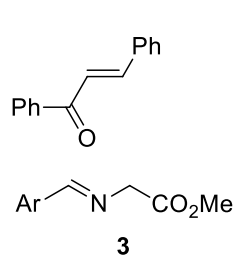

$\left[\left(S_{\mathrm{a}}\right) \text {-Binap-AuTFA }\right]_{2}$ $(5 \mathrm{~mol} \%)$
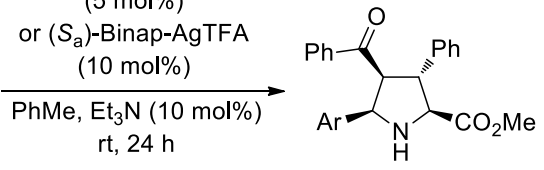

endo-15

b)

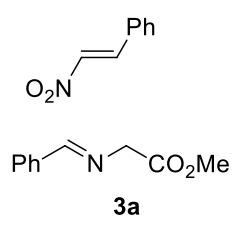

$\left[\left(S_{\mathrm{a}}\right) \text {-Binap-AuTFA }\right]_{2}$ $(5 \mathrm{~mol} \%)$

or $\left(S_{\mathrm{a}}\right)$-Binap-AgTFA (10 $\mathrm{mol} \%)$

PhMe, $\mathrm{Et}_{3} \mathrm{~N}(10 \mathrm{~mol} \%)$ $\mathrm{rt}, 24 \mathrm{~h}$

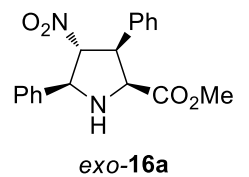

Scheme 5. $[(S) \text {-Binap-AuTFA }]_{2}$ versus $(S)$-Binap-AgTFA Catalyzed 1,3-dipolar cycloaddition of iminoesters 3 and chalcone or $\beta$-nitrostyrene.

Table 4. 1,3-DC of iminoesters 3 with chalcone and $\beta$-nitrostyrene

\begin{tabular}{|c|c|c|c|c|c|c|}
\hline \multirow[b]{2}{*}{ Entry } & \multirow[b]{2}{*}{ Ar, 3} & \multirow[b]{2}{*}{ Product } & \multicolumn{2}{|c|}{ Gold catalysis } & \multicolumn{2}{|c|}{ Silver catalysis } \\
\hline & & & $\begin{array}{l}\text { Yield } \\
(\%)^{[a][b]}\end{array}$ & $\begin{array}{l}e e \\
(\%)^{[\mathrm{c}]}\end{array}$ & $\begin{array}{l}\text { Yield } \\
(\%)^{[\mathrm{a}] \mathrm{b}]}\end{array}$ & $\begin{array}{l}e e \\
(\%)^{[\mathrm{c}] \mathrm{d}]}\end{array}$ \\
\hline 1 & $\mathrm{Ph}, \mathbf{3 a}$ & $15 \mathrm{a}$ & 95 & 80 & 80 & 20 \\
\hline 2 & $\begin{array}{l}\text { 2-Naphthyl, } \\
\text { 3b }\end{array}$ & $15 b$ & 90 & 60 & 81 & 50 \\
\hline 3 & $4-\mathrm{MeC}_{6} \mathrm{H}_{4}, \mathbf{3 f}$ & $15 f$ & 80 & 80 & 80 & 74 \\
\hline 4 & $\mathrm{Ph}, \mathbf{3 a}$ & $16 \mathrm{a}$ & $78^{[\mathrm{e}]}$ & 70 & $-[\mathrm{f}]$ & - \\
\hline 5 & $\mathrm{Ph}, \mathbf{3 a}^{[\mathrm{g}]}$ & $16 \mathrm{a}$ & $77^{[\mathrm{e}]}$ & 60 & $-[\mathrm{f}]$ & - \\
\hline
\end{tabular}

[a] Isolated yields after flash chromatography (silica gel). [b] The observed endo:exo

ratio was always $>98: 2$ ( ${ }^{1} \mathrm{H}$ NMR). [c] Determined by using analytical chiral HPLC columns. [d] Reaction performed with $\left(S_{\mathrm{a}}\right)$-Binap-AgTFA 6. [e] The observed endo:exo ratio was 20:80 ( ${ }^{1} \mathrm{H}$ NMR and using analytical chiral HPLC columns). [f] The reaction failed. [g] Reaction performed at $-20{ }^{\circ} \mathrm{C}$.

When conjugated esters were used as dipolarophiles, such as methyl or tert-butyl acrylate, dimethyl maleate, and diisopropyl fumarate, were allowed to react with inimoester $\mathbf{3 a}$, under the optimized reaction conditions, high yields of the corresponding endo-cycloadducts were obtained but with very low enantioselections.

The interest for identifying the catalytic species structure several analyses were carried out. The ${ }^{31} \mathrm{P}$ NMR analysis of $\left[\left(S_{\mathrm{a}}\right)\right.$-BinapAuTFA $]_{2}$ complex 2 revealed a singlet at $41.1 \mathrm{ppm}$, whereas the a singlet at $23.3 \mathrm{ppm}$ corresponded to $\left(S_{\mathrm{a}}\right)$-Binap-AuCl complex according to the literature. The dimeric complex is assumed to be a non-reactive complex but, in our case, the existence of the dipole would induce a reorganization of $\mathbf{2}$. For this study, the strongly coordinating thiazole-derived iminoester was selected. In the case of ESI-MS experiments peaks at $819,819.2$ and 819.6 were observed on elution with a mixture of acetonitrile and water, demonstrating the existence of such a dimeric complex. However, apart from the 
already described $\mathrm{M}^{+}$at 819 , a very intense one was observed at 1053 , which was possibly originated by the formation of the cluster 17 involving two molecules of the corresponding dipole. Efforts to obtain crystals from the mixture of equimolar amounts of chiral complex and the dipole derived from thiazole in dichloromethane resulted unfruitful. This analysis also revealed the existence (in less proportion) of fragments and peaks originated by the analogous complex 18 coordinated just with one equivalent of the iminoester $\left(\mathrm{M}^{+}\right.$966.4).

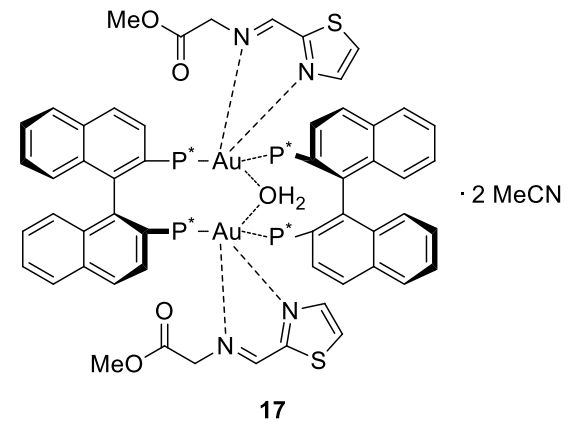

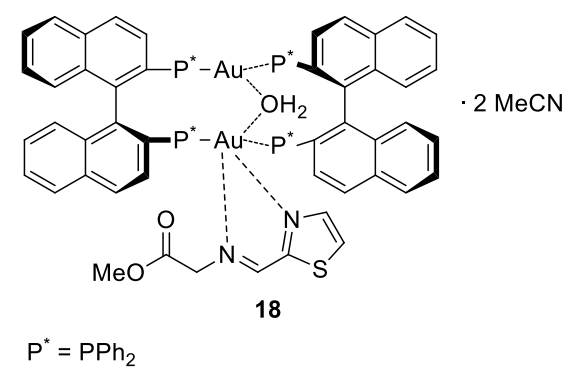

We observed a strong positive NLE (Figure 1) when the reaction of iminoester 3a was allowed to react with NMM employing different enantiomeric purity of the catalytic chiral complex 2 (X = TFA). This behavior can be justified by a generation of a reservoir of unproductive non-chiral heterodimer complex, ${ }^{[13]}$ increasing the concentration of the chiral catalytic active species in solution.

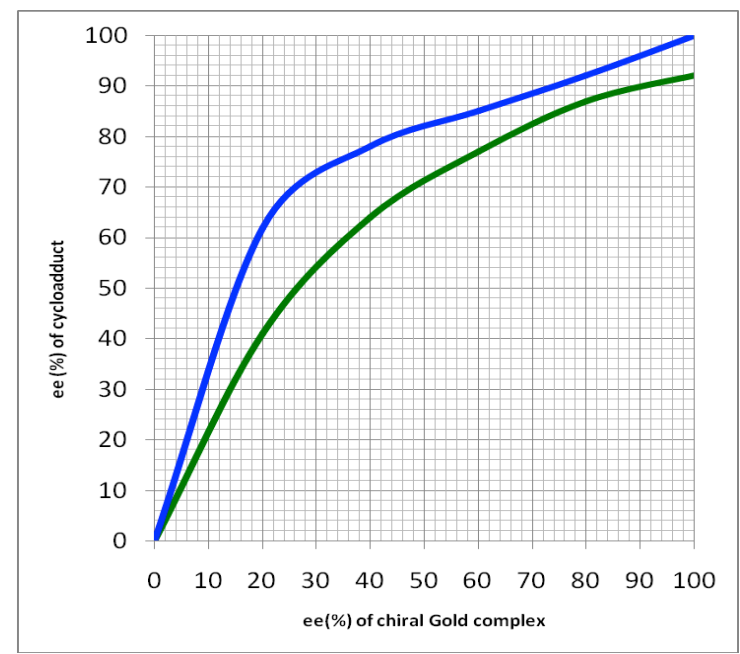

Figure 1. Experimental (blue line) and theoretical (green line) NLE observed in the chiral complex 2-catalyzed 1,3-DC between the iminoester 3a and NMM.

Next, we performed DFT calculations to shed light on the origins of the great stereocontrol obtained in these $\left(S_{\mathrm{a}}\right)$-Binap $\mathrm{Au}(\mathrm{I})$ catalyzed 1,3-DC reactions. In a previous work, we demonstrated that the stereoselectivity obtained employing chiral ligandmetallated azomethine ylide in 1,3-DC is a consequence of the blockage of one of the ylide stereogenic faces by the chiral ligand. ${ }^{[14]}$

We calculated the geometries of the most stable $\left(S_{\mathrm{a}}\right)$-Binap silver(I) or gold(I) ylide I reactive complexes (Figure 2). As expected, in the case of the silver complex (Figure 2A) the metallic centre adopts a tetrahedral environment. This $\mathrm{Ag}(\mathrm{I})$ is surrounded by both phosphorous atoms of the ligand and by the nitrogen and oxygen atoms of the ylide (Figure 2A). Due to this coordination pattern, the distance between the chiral ligand and the prochiral faces is short enough to induce a proper stereoselectivity allowing the attack of the dipolarophile only by the $(2 \mathrm{Si}, 5 \mathrm{Re})$ face of the ylide. This is in good agreement with the experimental evidence of the major formation of the $(S, S, S, R)$ cycloadducts. ${ }^{[\mathrm{b}]}$

In the case of monomeric $\left(S_{\mathrm{a}}\right)$-Binap-Au-ylide $\mathbf{I}$ complex (Figure 2B), the gold atom presents a linear coordination and it is surrounded only by one phosphorous atom of the ligand and the nitrogen atom of the ylide. In this case, the carboxy-gold distance is c.a. $0.3 \AA$ larger than the carboxy-silver distance as one may expect from the known low oxophilicity of the gold(I) cation. This geometry makes the two prochiral faces approximately equivalent. Therefore, no significant enantioselection should be expected in the $[3+2]$ reaction.

We also considered the existence of $\left(S_{\mathrm{a}}\right)$-Binap-Au dimeric units as the actual catalytic complex (Figures $2 \mathrm{~B}$ and $2 \mathrm{C}$ ). Under these conditions only two significant conformations are energetically accessible. In $\left[\left(S_{\mathrm{a}}\right) \text {-Binap-Au }\right]_{2}$-ylide-I only the $(2 \mathrm{Si}, 5 \mathrm{Re})$ face is accessible. By contrast, in $\left[\left(S_{\mathrm{a}}\right) \text {-Binap-Au }\right]_{2}$-ylide-I-b the accessible face is the opposite one [named $(2 R e, 5 \mathrm{Si})]$. However, the difference in energy of the less stable one is enough to assume that only $\mathbf{I}$ is present in solution at room temperature. Therefore, the major product corresponds to the $(S, S, S, R)$ cycloadducts. In previous works focused on catalyzed 1,3-DC of azomethine ylides and maleimides we demonstrated the exclusive preference of the reaction for endo-cycloadducts. Therefore, only this approach will be considered in the following study. ${ }^{[8 b]}$

The main geometric features and relative energies of the stationary points corresponding to the 1,3-DC of (S)-Binap-Au-ylide dimers are gathered in Figure 3. As expected from the previous analysis, endo-saddle points corresponding to the reaction of the dipolarophile through the hindered $(2 R e, 5 S i)$ face of ylide $\left[\left(S_{a}\right)\right.$ BinapAu $]_{2}$ I are of much higher energy than the obtained from suprafacial approach through $(2 \mathrm{Si}, 5 \mathrm{Re})$ of ylide $\left[\left(S_{a}\right) \text {-BinapAu }\right]_{2} \mathbf{I}-\mathbf{- b}$ (see the supporting information for further details). Our calculations also indicate that the reaction presents a concerted but slightly asynchronous transition structure in which the critical distance of the forming $\mathrm{C} 2-\mathrm{C}_{\mathrm{nmm}}$ bond is shorter than that associated with formation of the $\mathrm{C} 5-\mathrm{C}^{\prime}{ }_{\mathrm{nmm}}$ bond.

The activation Gibbs free energy barrier associated with the formation of of $(S, S, S, R)$-II is c.a. $4.5 \mathrm{kcal} \mathrm{mol}^{-1}$ higher than the one associated with the formation of the corresponding enantiomer. If we assume a preequilibrium between both possible reactive ylides, Curtin-Hammet kinetics show that the product ratio should depend on the free Gibbs activation energy difference of both corresponding transition structures. Under this kinetic framework, our calculations predict the major formation of $(S, S, S, R)$-II with ee $90 \%$ c.a. in good agreement with the experimental results.

The formation of the major complexed cycloadduct is thermodynamically favored (c.a. $6 \mathrm{kcal} \mathrm{mol}^{-1}$, Figure 3). The catalytic cycle is completed because the final step $(\mathbf{3 i}+(S, S, S, R)-\mathbf{I I}$ to release the cycloadduct $\mathbf{5 i})$ is endothermic $\left(3 \mathrm{kcal} \mathrm{mol}^{-1}\right)$ and the recovery of the reactive ylide is ensured (Scheme 6). 


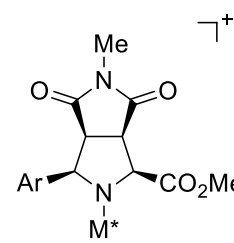

$(S, S, S, R)-$ II

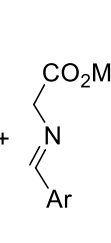

3

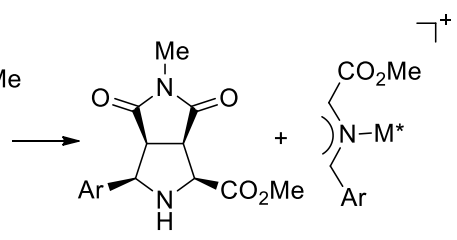

5
$\left[\left(S_{\mathrm{a}}\right)-\text { Binap-Au }\right]_{2}-1$

$\mathrm{M}^{*}=\left[\mathrm{Au}-\left(\mathrm{S}_{\mathrm{a}}\right)-\mathrm{Binap}\right]_{2}$

$\mathrm{Ar}=p-\mathrm{OMePh}$

$\mathrm{G}_{\mathrm{rxn}}=-2.8 \mathrm{kcal} \mathrm{mol}^{-1}$

Scheme 6. Last step in the catalytic cycle to generate compound 5.

Finally we studied computationally the strong positive NLE observed in the reaction of the iminoester $\mathbf{3 a}$ and NMM catalyzed by gold(I) complexes. We calculated the stability of the possible azomethine ylide complexes with hetero Binap-gold(I) dimers. Our results indicate that there is only one energetically feasible geometry for the $\left[\left(S_{a}\right)\right.$-Binap, $\left(R_{a}\right)$-Binap-Au $\left.\mathrm{Au}_{2}\right]-\mathbf{I}$ reactive complex and this complex is much more stable than the possible homodimeric species. Our results also indicate that all of the $\left(R_{\mathrm{a}}\right)$-Binap present in solution would be involved in the generation of the heterodimeric form. Therefore the presence of $\left[\left(R_{\mathrm{a}}\right)-\mathrm{Binap}-\mathrm{Au}\right]_{2}-\mathbf{I}$ complex can be neglected.

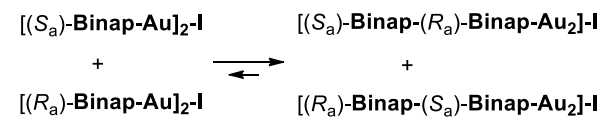

$$
\text { 国 } \mathrm{G}_{\mathrm{rxn}}=-16.2 \mathrm{kcal} \mathrm{mol}^{-1}
$$

Scheme 7. Equilibrium between all the possible reactive dimeric gold (I) azomethine ylide complexes when non-enantiopure Binap ligand is employed.

We also studied the reaction mechanism when the heterochiral gold(I) complexes are involved. The calculated activation free energy barrier associated with the reaction of $\left[\left(S_{\mathrm{a}}\right)\right.$-Binap, $\left(R_{\mathrm{a}}\right)$ Binap- $\left.\mathrm{Au}_{2}\right]-\mathrm{I}$ and NMM is $20.2 \mathrm{kcal} \mathrm{mol}{ }^{-1}$, c. a. $1 \mathrm{kcal} \mathrm{mol}^{-1}$ higher than the value associated with the reaction of $\left[\left(S_{\mathrm{a}}\right) \text {-Binap-Au }\right]_{2}-\mathbf{I}$ complex (see supporting information for further geometrical details of the transition structure).

$$
\begin{aligned}
& {\left[\left(S_{\mathrm{a}}\right)-\text {-BinapAu }\right]_{2}-\mathrm{I}+4 \mathrm{a} \stackrel{k_{\text {номо }}}{\longrightarrow}(S, S, S, R)-\mathrm{II} \stackrel{+3}{\longrightarrow} 5+\mathrm{M}_{\mathrm{i}}{ }^{*}-\mathrm{I}}
\end{aligned}
$$

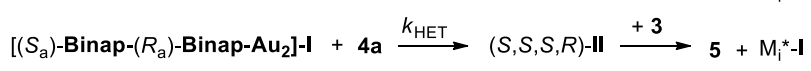

$$
\begin{aligned}
& {\left[\left(R_{\mathrm{a}}\right) \text {-Binap- }\left(S_{\mathrm{a}}\right)-\text { Binap-Au } 2\right]-\mathrm{I}+4 \mathrm{a} \stackrel{k_{\mathrm{HET}}}{\longrightarrow}(R, R, R, S)-\mathrm{II} \stackrel{+3}{\longrightarrow} 5_{\mathrm{ent}^{+}} \mathrm{M}_{\mathrm{i}}^{*}-\mathrm{I}}
\end{aligned}
$$

Scheme 8. Reaction framework for the reaction of chiral complex 2-catalyzed 1,3-DC between the iminoester 3a and NMM when non-enantiopure Binap ligand is employed.

The kinetic constants of the [3+2] cycloaddition process within the framework indicated in Scheme 8 have been calculated employing the Eyring equations and using the previously computed activation free energy barriers. We have analysed the kinetic system employing numerical integration assuming the total recovery of the catalyst and the irreversibility of the process. Within this framework the expected stereochemical outcome of the reaction employing different non-enantiopure catalyst mixtures shown in Figure 1 (vide supra) was surveyed. Our calculations show that the generation of the heterodimer produces less reactive complexes, which avoids the formation of the non-desired homodimer $\left[\left(R_{a}\right) \text {-BinapAu }\right]_{2} \mathbf{I}$. Therefore an increase of the ee of the cycloadduct is observed. Our results are in qualitative agreement with the experimental evidences (Figure 1).

\section{Conclusion}

The enantioselective 1,3-DC of azomethine ylides and electrophilic alkenes has been successfully catalysed by $\left[\left(S_{\mathrm{a}}\right) \text {-Binap-AuTFA }\right]_{2}$ complex. The results obtained with this catalyst improved the analogous ones described for the reactions carried out in the presence of $\left(S_{\mathrm{a}}\right)$-Binap-AgTFA, especially when a hindered component (dipole or dipolarophile) are involved. In some examples, $\left[\left(S_{\mathrm{a}}\right) \text {-Binap-AuTFA }\right]_{2}$ catalyst, once ligaed to the 1,3-dipole, acted as a bifunctional complex activating the dipolarophile and as an internal base. Even unreactive dipolarohiles under silver catalysis, such as nitrostyrene, were appropriate under the gold(I) catalysis. The origin of the enantioselection was confirmed by DFT calculations, revealing very interesting geometric data of the transition states. Computational studies of the strong positive NLE showed important differences between all of the possible catalytic species demonstrating the existance of very stable and unreactive heterodiemric complexes. Finally, regarding the observed positive NLE, calculations predicted a very close NLE plot with respect to the experimental data. To the best of our knowledge this is the first work in which NLE is analysed using computational tools.

\section{Experimental Section}

General. All reactions were carried out in the absence of light. Anhydrous solvents were freshly distilled under an argon atmosphere. Aldehydes were also distilled prior to use for the elaboration of the iminoesters. Melting points were determined with a Reichert Thermovar hot plate apparatus and are uncorrected. Only the structurally most important peaks of the IR spectra (recorded on a Nicolet 510 P-FT and on a Jasco FTIR 4100 ) are listed. ${ }^{1} \mathrm{H}$ NMR (300 MHz) and ${ }^{13} \mathrm{C}$ NMR (75 MHz) spectra were obtained on a Bruker AC-300 using $\mathrm{CDCl}_{3}$ as solvent and TMS as internal standard, unless otherwise stated. Optical rotations were measured on a Perkin Elmer 341 polarimeter. HPLC analyses were performed on a JASCO 2000-series equipped with a chiral column (detailed for each compound in the main text), using mixtures of $n$-hexane/isopropyl alcohol as mobile phase, at $25{ }^{\circ} \mathrm{C}$. Low-resolution electron impact (EI) mass spectra were obtained at $70 \mathrm{eV}$ on a Shimadzu QP-5000 and high-resolution mass spectra were obtained on a Finnigan VG Platform. HRMS (EI) were recorded on a Finnigan MAT 95S. Microanalyses were performed on a Perkin Elmer 2400 and a Carlo Erba EA1108. Analytical TLC was performed on Schleicher \& Schuell F1400/LS silica gel plates and the spots were visualized under UV light $(\lambda=254 \mathrm{~nm})$. For flash chromatography we employed Merck silica gel $60(0.040-0.063 \mathrm{~mm})$. Complexes 1, and 2 were prepared according to the reported procedure. ${ }^{[12]}$ Silver complex was ready prepared just by mixing equimolar amounts of the Binap chiral ligand and AgTFA. All of the transformations performed with this catalyst were performed in the absence of light.

Hybrid QM/MM calculations for optimizations of saddle points were performed in terms of ONIOMc ${ }^{[15]}$ method implemented in GAUSSIAN09 suite of programs. ${ }^{[16]}$ Ball $\&$ stick model in Figure 2 shows atoms included in the high-level layer, and a wire model is used to represent atoms included in the low-level layer. In the high level layer, the electron correlation was partially taken into account by using the hybrid functional B3LYP ${ }^{[17]}$ combined with Hay-Wadt small core effective potential $(\mathrm{ECP})^{[18]}$ basis set. $\mathrm{UFF}^{[19]}$ molecular mechanics force field was employed in the low-level layer. This combination has offered excellent results in studies of organocatalytic mechanisms. ${ }^{[20]}$ Thermal corrections of Gibbs free energies were computed at the same level of theory and were not scaled. All stationary points were characterized by harmonic analysis. Reactant intermediates and cycloadducts have positive definite Hessian matrices. Transition structures show only one negative eiganvalue in their diagonalysed force constant matrices, and their associated eigenvectors were confirmed to correspond to the motion along the reaction coordinate under consideration.

1,3-Dipolar cycloaddition of iminoesters 3 or 7 and dipolarophiles. General procedure. To a solution of the in situ prepared chiral gold complex or chiral silver complex $(0.05 \mathrm{mmol})$ in toluene $(2 \mathrm{~mL})$ was added, at room temperature, a solution of the iminoester $(0.5 \mathrm{mmol})$ and dipolarophile $(0.5 \mathrm{mmol})$ in toluene $(2 \mathrm{~mL})$. In some cases DIPEA or triethylamine $(0.05 \mathrm{mmol})$ was added (see, Tables) and the mixture stirred at room temperature for $16-48 \mathrm{~h}$ (see, Tables). The reaction was filtered off through a celite pad and the organic filtrate was directly evaporated and the residue was purified by recrystallization or by flash chromatography yielding pure endocycloadducts $5,8,10,15$ or 16 .

Methyl (IS,3R,3aS,6aR)-5-methyl-3-phenyl-4,6-dioxooctahydropyrrolo[3,4-c]pyrrole1-carboxylate 5aa. ${ }^{[21][22]}$ 
Methyl (1S,3R,3aS,6aR)-5-ethyl-3-phenyl-4,6-dioxooctahydropyrrolo[3,4-c]pyrrole-1carboxylate 5ab. ${ }^{[21][22]}$

Methyl (IS,3R,3aS,6aR)-3,5-diphenyl-4,6-dioxooctahydropyrrolo[3,4-c]pyrrole-1carboxylate 5 ac $^{[21,22]}$

Methyl (IS,3R,3aS,6aR)-5-methyl-3-(2-naphthyl)-4,6-dioxooctahydropyrrolo[3,4c]pyrrole-1-carboxylate 5 ba ${ }^{[8]}$

Methyl (IS,3R,3aS,6aR)-5-ethyl-3-(2-naphthyl)-4,6-dioxooctahydropyrrolo[3,4c]pyrrole-1-carboxylate 5bb: Colourless solid; mp: 196-197 ${ }^{\circ} \mathrm{C}$ ( $n$-hexane/ethyl acetate); $[\alpha]_{\mathrm{D}}{ }^{20}=7.0\left(c 0.5, \mathrm{CH}_{2} \mathrm{Cl}_{2}, 50 \%\right.$ ee by HPLC); IR (neat) $v_{\max }: 3330,2946$, and $1748 \mathrm{~cm}^{-1}$; ${ }^{1} \mathrm{H}$ NMR $\delta_{\mathrm{H}}: 1.05\left(\mathrm{t}, J=7.2 \mathrm{~Hz}, 1 \mathrm{H}, \mathrm{CH}_{2} \mathrm{CH}_{3}\right), 2.45(\mathrm{~s}, 1 \mathrm{H}, \mathrm{NH}), 3.39(\mathrm{~m}$, $\left.2 \mathrm{H}, \mathrm{CH}_{2}\right), 3.47\left(\mathrm{dd}, J=7.6\right.$, and $\left.8.0 \mathrm{~Hz}, 1 \mathrm{H}, \mathrm{CHCCO}_{2}\right), 3.55(\mathrm{dd}, J=7.0$ and $8.0 \mathrm{~Hz}$ $1 \mathrm{H}, \mathrm{CHCCAr}), 3.91\left(\mathrm{~s}, 3 \mathrm{H}, \mathrm{OCH}_{3}\right), 4.08\left(\mathrm{dd}, J=7.0\right.$, and $\left.5.2 \mathrm{~Hz}, 1 \mathrm{H}, \mathrm{CHCO}_{2}\right), 4.63$ (dd, $J=7.6$, and $5.2 \mathrm{~Hz}, 1 \mathrm{H}, \mathrm{CHAr}$ ), $7.46(\mathrm{dd}, J=6.2,3.3 \mathrm{~Hz}, 3 \mathrm{H}, \mathrm{Ar} H$ ), $7.95-7.65$ $(\mathrm{m}, 4 \mathrm{H}, \mathrm{Ar} H) ;{ }^{13} \mathrm{C}$ NMR $\delta_{\mathrm{C}}: 13.1\left(\mathrm{CH}_{3} \mathrm{CH}_{2}\right), 33.9\left(\mathrm{CH}_{3}\right), 48.1,49.1,52.3(2 \mathrm{xCHCON}$ and $\left.\mathrm{CO}_{2} \mathrm{CH}_{3}\right), 61.6,63.9\left(\mathrm{CHCO}_{2} \mathrm{Me}\right), 67.9(\mathrm{ArCHN}), 125.3,125.4,125.9,126.1$, 126.2, 127.8, 127.9, 133.2, 134.2 and 134.2 ( $\mathrm{ArC}), 170.15,174.33,175.70(3 \mathrm{xCO})$; MS (EI) $m / z$ (\%): 352 (20), 293 (45), 127 (34), 91 (100); HRMS for $\mathrm{C}_{20} \mathrm{H}_{20} \mathrm{~N}_{2} \mathrm{O}_{4}$ require 352.3838; found 352.3830; HPLC (Chiralpak AS), $1 \mathrm{~mL} n$-hexane $/ i-\mathrm{PrOH}: 20 / 80, \lambda$ $225 \mathrm{~nm}, \mathrm{t}_{\text {maj }}=7.4 \mathrm{~min}, \mathrm{t}_{\min }=20 \mathrm{~min}$

Methyl (1S,3R,3aS,6aR)-3-(2-naphthyl)-5-phenyl-4,6-dioxooctahydropyrrolo[3,4c]pyrrole-1-carboxylate 5 bc ${ }^{[7 \mathrm{f]}}$

Methyl (1S,3R,3aS,6aR)-5-methyl-4,6-dioxo-3-o-tolyloctahydropyrrolo[3,4-c]pyrrole-1carboxylate $5 \mathrm{ca}^{\left[{ }^{[8]}\right.}$

Methyl (IS,3R,3aS,6aR)-3-(2-chlorophenyl)-5-methyl-4,6-dioxooctahydropyrrolo[3,4c]pyrrole-1-carboxylate $5 \mathbf{d a}{ }^{[8]}$

Methyl (1S,3R,3aS,6aR)-5-methyl-3-(4-methylphenyl)-4,6-dioxooctahydropyrrolo[3,4c]pyrrole-1-carboxylate 5ea ${ }^{[8]}$

Methyl (1S,3R,3aS,6aR)-3-(4-methoxyphenyl)-5-methyl-4,6-dioxooctahydropyrrolo[3,4c]pyrrole-1-carboxylate $5 \boldsymbol{f a}^{\left[{ }^{[8]}\right.}$

Methyl (1S,3R,3aS,6aR)-3-(4-chlorophenyl)-5-methyl-4,6-dioxooctahydropyrrolo[3,4c]pyrrole-1-carboxylate 5 ga ${ }^{[8]}$

Methyl (IS,3R,3aS,6aR)-1-benzyl-5-methyl-4,6-dioxo-3-phenyloctahydropyrrolo[3,4c]pyrrole-1-carboxylate $8 \mathbf{a a}^{[8]}$

Methyl $(2 R, 3 R, 4 R, 5 S)-5$-phenyl-3,4-bis(phenylsulfonyl)pyrrolidine-2-carboxylate 10a: Colourless viscous oil; $[\alpha]_{\mathrm{D}}{ }^{20}=+1.2^{\circ}\left(c 1.0, \mathrm{CH}_{2} \mathrm{Cl}_{2}, 90 \%\right.$ ee by HPLC); IR (neat) $v_{\max }$ : 3311, and $1753 \mathrm{~cm}^{-1} ;{ }^{1} \mathrm{H}$ RMN $\delta_{\mathrm{H}}: 3.00$ (br. s, $\left.1 \mathrm{H}, \mathrm{NH}\right) 3.68$ (s, $3 \mathrm{H}, \mathrm{CH}_{3}$ ); 4,33 (br. s, $1 \mathrm{H}, \mathrm{NCHCO}), 4.41,4.47$ (2xdd, $J=2.4$ and $6.3 \mathrm{~Hz}, 2 \mathrm{H}, 2 \mathrm{xCHS}), 4.64(\mathrm{~m}, 1 \mathrm{H}, \mathrm{PhCHN})$ 7.28-7.26 (m, 2H, $\operatorname{Ar} H$ ), 7.34-7.32 (m, 2H, $\operatorname{ArH}), 7.46-7.42(\mathrm{~m}, 2 \mathrm{H}, \mathrm{Ar} H), 7.61-7.52(\mathrm{~m}$ $3 \mathrm{H}, \mathrm{ArH}), 7.72-7.65(\mathrm{~m}, 5 \mathrm{H}, \mathrm{ArH}),{ }^{13} \mathrm{C} \mathrm{RMN} \delta_{\mathrm{C}}: 37.4\left(\mathrm{OCH}_{3}\right), 48.6(\mathrm{CHCO}), 49.1$, 52.7 (2xCHS), $56.0(\mathrm{CHPh}), 112.3,112.9,113.2,113.4,113.7,113.9,114.1,114.2$ $119.2,121.9,122.8,123.1$ (Ar), $152.4\left(\mathrm{CO}_{2}\right)$; MS (EI) $m / z(\%): 485\left(\mathrm{M}^{+},<1 \%\right), 284$ (11), 203 (15), 202 (100), 170 (18), 144 (27), 143 (60), 115 (14), 77 (14); HRMS for $\mathrm{C}_{24} \mathrm{H}_{23} \mathrm{NO}_{6} \mathrm{~S}_{2}$ requires: 485.5725 ; found: 485.5717 ; HPLC (Chiralpak IA), $1 \mathrm{~mL} / \mathrm{min}, n$ hexane $/ i-\mathrm{PrOH}: 80 / 20, \lambda 224 \mathrm{~nm}, \mathrm{t}_{\mathrm{maj}}=50.23 \mathrm{~min}, \mathrm{t}_{\min }=30.92 \mathrm{~min}$

Methyl (2R,3R,4R,5S)-5-(naphth-2-yl)-3,4-bis(phenylsulfonyl)pirrolidine-2-carboxylate 10b: Colluorless viscous oil; $[\alpha]_{\mathrm{D}}{ }^{20}=+17.1^{\circ}\left(c 0.5, \mathrm{CH}_{2} \mathrm{Cl}_{2}, 92 \%\right.$ ee by HPLC); IR (neat) $v_{\max }: 3340,1741.45 \mathrm{~cm}^{-1} ;{ }^{1} \mathrm{H}$ RMN $\delta_{\mathrm{H}}: 3.15$ (br. s, $\left.1 \mathrm{H}, \mathrm{NH}\right), 3.69\left(\mathrm{~s}, 3 \mathrm{H}, \mathrm{CH}_{3}\right)$ 4.38 (br. s, $1 \mathrm{H}, \mathrm{NCHCO}), 4.45$ (dd, $J=2.4 \mathrm{~Hz}, 6.4 \mathrm{~Hz}, 1 \mathrm{H}, \mathrm{CHS}), 4.49$ (dd, $J=2.4 \mathrm{~Hz}$, $6.0 \mathrm{~Hz}, 1 \mathrm{H}, \mathrm{CHS}), 4.79$ (br. s, $1 \mathrm{H}, \mathrm{PhC} H \mathrm{~N}), 7.39-7.35$ (m, 2H, $\mathrm{Ar} H), 7.56-7.45(\mathrm{~m}, 6 \mathrm{H}$ $\operatorname{Ar} H)$, 7.72-7.60 (m, 7H, $\operatorname{Ar} H), 7.81-7.77(\mathrm{~m}, 2 \mathrm{H}, \operatorname{Ar} H) ;{ }^{13} \mathrm{C} \mathrm{RMN} \delta_{\mathrm{C}}: 38.5\left(\mathrm{OCH}_{3}\right)$, 48.6 (CHCO), 49.1, 52.7 (2xCHS), $55.9(\mathrm{ArCH}), 109.3,109.4,111.2,111.3,112.0$ $112.3,112.4,112.5,113.0,113.3,113.4,113.5,113.8,114.0,114.2,114.3,119.3,119.2$ $(\mathrm{ArC}), 152.5\left(\mathrm{CO}_{2}\right)$; MS (EI) $m / z(\%): 535\left(\mathrm{M}^{+},<1 \%\right), 394$ (26), $392(11), 391$ (35), 359 (28), 268 (21), 267 (19), 266 (17), 253 (19), 252 (100), 251 (31), 250 (13), 220 (31) 219 (31), 194 (35), 193 (62), 192 (22), 191 (31), 190 (41), 167 (15), $166(11), 165$ (47) 164 (13), 163 (15), 155 (11), 153 (11), 152 (22), 142 (11), 127 (17), 125 (14), 78 (20), 77 (46), 51 (15); HRMS for $\mathrm{C}_{28} \mathrm{H}_{25} \mathrm{NO}_{6} \mathrm{~S}_{2}$ requires: 535.6312; found: 535.6315; HPLC (Chiralpak AD), $1 \mathrm{~mL} / \mathrm{min}, n$-hexane $/ i$-PrOH: $75 / 25, \lambda 227 \mathrm{~nm}, \mathrm{t}_{\mathrm{maj}}=57.01 \mathrm{~min}, \mathrm{t}_{\min }=$ $51.97 \mathrm{~min}$

Methyl (2R,3R,4R,5S)-3,4-bis(phenylsulfonyl)-5-(pyrid-3-yl)pirrolidine-2-carboxylate 10e: Pale yellow oil; $[\alpha]_{\mathrm{D}}{ }^{20}=-63.9^{\circ}\left(c 1.2, \mathrm{CH}_{2} \mathrm{Cl}_{2}, 92 \%\right.$ ee by HPLC); IR (neat) $v_{\max }$ : $3310,1753 \mathrm{~cm}^{-1}$; ${ }^{1} \mathrm{H}$ RMN $\delta_{\mathrm{H}}: 1.90$ (br. s, $\left.1 \mathrm{H}, \mathrm{NH}\right), 3.68$ (s, $\left.3 \mathrm{H}, \mathrm{CH}_{3}\right) ; 4.33$ (dd, $J=2.3$ $\mathrm{Hz}, 6.4 \mathrm{~Hz}, 1 \mathrm{H}, \mathrm{CHS}), 4.35$ (m, 1H, NCHCO), 4.43 (dd, $J=2.3 \mathrm{~Hz}, 5.9 \mathrm{~Hz}, 1 \mathrm{H}, \mathrm{CHS}$ ), $4.66(\mathrm{~d}, J=6.2 \mathrm{~Hz}, 1 \mathrm{H}, \operatorname{ArC} H \mathrm{~N}), 7.46(\mathrm{t}, J=7.7 \mathrm{~Hz}, 8.0 \mathrm{~Hz}, 2 \mathrm{H}, \operatorname{Ar} H), 7.53(\mathrm{t}, J=7.4$ $\mathrm{Hz}, 8.2 \mathrm{~Hz}, 2 H, \mathrm{ArH}), 7.61-7.65(\mathrm{~m}, 2 \mathrm{H}, \operatorname{Ar} H), 7.70(\mathrm{t}, J=7.3 \mathrm{~Hz}, 7.9 \mathrm{~Hz}, 6 \mathrm{H}, \operatorname{Ar} H)$ $8.36(\mathrm{~s}, 1 \mathrm{H}, \mathrm{Ar} H), 8.52(\mathrm{~s}, 1 \mathrm{H}, \mathrm{Ar} H) ;{ }^{13} \mathrm{C} \mathrm{RMN} \delta_{\mathrm{C}}: 34.6\left(\mathrm{OCH}_{3}\right), 45.8(\mathrm{CHCO}), 48.9$ 52.4, (2xCHS), 55.7 ( $\mathrm{ArCH}), 108.7$ 113.3, 113.4, 114.3, 114.6, 119.3, 119.5, 119.6 119.8, 121.5, 122.5, 134.0, 134.7 ( $\mathrm{ArC}), 152.3\left(\mathrm{CO}_{2} \mathrm{Me}\right)$; MS (EI) $m / z(\%): 486\left(\mathrm{M}^{+},<\right.$ 1\%), 285 (10), 204 (14), 203 (100), 171 (10), 145 (19), 144 (30), 77 (18); HRMS for $\mathrm{C}_{28} \mathrm{H}_{25} \mathrm{NO}_{6} \mathrm{~S}_{2}$ requires: 486.5606; found: 486.5601; HPLC (Chiralpak AD), $1 \mathrm{~mL} / \mathrm{min}$, $n$-hexane $/ i$-PrOH: 50/50, $\lambda 222 \mathrm{~nm}, \mathrm{t}_{\mathrm{maj}}=15.05 \mathrm{~min}, \mathrm{t}_{\mathrm{min}}=7.91 \mathrm{~min}$.

Methyl (2R,3R,4R,5S)-5-(4-methylphenyl)-3,4-bis(phenylsulfonyl)pyrrolidine-2 carboxylate 10f: Colourless viscous oil; $[\alpha]_{\mathrm{D}}{ }^{20}=+2.3^{\circ}(c) 1.0, \mathrm{CH}_{2} \mathrm{Cl}_{2}, 88 \%$ ee by HPLC); IR (neat) $v_{\max }: 3341,1747 \mathrm{~cm}^{-1} ;{ }^{1} \mathrm{H} \mathrm{RMN} \delta_{\mathrm{H}}: 2.30\left(\mathrm{~s}, 3 \mathrm{H}, \mathrm{ArCH}_{3}\right) ; 2.95$ (br. s, $1 \mathrm{H}, \mathrm{NH}), 3.66\left(\mathrm{~s}, 3 \mathrm{H}, \mathrm{OCH}_{3}\right), 4.31-4.27(\mathrm{~m}, 1 \mathrm{H}, \mathrm{CHCO}), 4.35$ (dd, $J=2.4 \mathrm{~Hz}, 6.4 \mathrm{~Hz}$, $1 \mathrm{H}, \mathrm{CHS}$ ), 4.41 (dd, $J=2.4 \mathrm{~Hz}, 6.0 \mathrm{~Hz}, 1 \mathrm{H}, \mathrm{CHS}$ ), 4.61-4.57 (br. s, $1 \mathrm{H}, \operatorname{ArCHN}$ ), 7.06 $(\mathrm{d}, J=8.1 \mathrm{~Hz}, 1 \mathrm{H}, \operatorname{Ar} H), 7.20(\mathrm{~d}, J=8.1 \mathrm{~Hz}, 1 \mathrm{H}, \operatorname{Ar} H), 7.54-7.40(\mathrm{~m}, 6 \mathrm{H}, \operatorname{Ar} H), 7.64$ $7.57(\mathrm{~m}, 2 \mathrm{H}, \mathrm{Ar} H), 7.70-7.66(\mathrm{~m}, 4 \mathrm{H}, \mathrm{Ar} H) ;{ }^{13} \mathrm{C} \mathrm{RMN} \delta_{\mathrm{C}}: 21.2\left(\mathrm{CH}_{3} \mathrm{Ph}\right) 53.6\left(\mathrm{OCH}_{3}\right)$ 63.5 (CHCO), 64.267 .9 (2xCHS), 71.2 ( $\mathrm{ArCHN}), 127.3$ 128.5, 128.6, 129.1, 129.2,
129.3, 129.4, 129.5, 134.3, 135.0, 138.1, $138.2(\mathrm{ArC}), 167.6(\mathrm{CO}) ;(\mathrm{EI}) \mathrm{m} / \mathrm{z}(\%): 499$ $\left(\mathrm{M}^{+},<1 \%\right), 358$ (24), 217 (15), 216 (100), 184 (22), 158 (22), 157 (48), 156 (14), 77 (15); HRMS for $\mathrm{C}_{25} \mathrm{H}_{25} \mathrm{NO}_{6} \mathrm{~S}_{2}$ requires: 499.5991; found: 499.6000; HPLC (Chiralpak AD), $1 \mathrm{~mL} / \mathrm{min}, n$-hexane $/ i$-PrOH: $75 / 25, \lambda 220 \mathrm{~nm}, \mathrm{t}_{\text {maj }}=50.08 \mathrm{~min}, \mathrm{t}_{\min }=26.11 \mathrm{~min}$.

Methyl (2R,3R,4R,5S)-5-(4-chlorophenyl)-3,4-bis(phenylsulfonyl)pyrrolidine-2carboxylate 10h: Colourless viscous oil; $[\alpha]_{\mathrm{D}}{ }^{20}=+91.6^{\circ}\left(c 1.0, \mathrm{CH}_{2} \mathrm{Cl}_{2}, 45 \%\right.$ ee by HPLC); IR (neat) $v_{\max }: 3341,1750 \mathrm{~cm}^{-1}$; ${ }^{1} \mathrm{H}$ RMN $\delta_{\mathrm{H}}: 2.90$ (deform. t, $\left.1 \mathrm{H}, \mathrm{NH}\right), 3.67$ (s, $\left.3 \mathrm{H}, \mathrm{CH}_{3}\right) ; 4.30$ (dd, $J=2.3 \mathrm{~Hz}, 6.3 \mathrm{~Hz}, 1 \mathrm{H}, \mathrm{CHS}$ ), 4.34 (br. s, 1H, CHCO), 4.38 (dd $J=2.3 \mathrm{~Hz}, 5.9 \mathrm{~Hz}, 1 \mathrm{H}, \mathrm{CHS}), 4.66-4.61(\mathrm{~m}, 1 \mathrm{H}, \operatorname{ArCHN}), 7.30-7.21(\mathrm{~m}, 3 \mathrm{H}, \operatorname{Ar} H)$, 7.53-7.43 (m, 4H, $\mathrm{Ar} H), 7.71-7.59(\mathrm{~m}, 7 \mathrm{H}, \mathrm{Ar} H) ;{ }^{13} \mathrm{C} \mathrm{RMN} \delta_{\mathrm{C}}: 37.5\left(\mathrm{OCH}_{3}\right), 45.2$ (CHCO), 48.9, 52.6 (2xCHS), $55.9(\mathrm{ArCH}), 113.2,113.7,113.8,114.0,114.1,114.3$, $114.5,114.8,119.4,119.5,121.7,123.0(\mathrm{ArC}), 152.3(\mathrm{CO})$; MS (EI) $m / z(\%): 519\left(\mathrm{M}^{+}\right.$ $<1 \%), 238$ (32), 237 (17), 236 (100), 235 (12), 204 (19), 203 (15), 179 (17), 178 (19), 177 (48), 143 (21), 140 (14), 125 (16), 115 (13), 77 (29), 152 (13); HRMS for $\mathrm{C}_{24} \mathrm{H}_{22} \mathrm{ClNO}_{6} \mathrm{~S}_{2}$ requires: 520.0176; found: 520.0170; HPLC (Chiralpak AD), $1 \mathrm{~mL} / \mathrm{min}$, $n$-hexane $/ i$-PrOH: $70 / 30, \lambda 220 \mathrm{~nm}), \mathrm{t}_{\text {maj }}=41.08 \mathrm{~min}, \mathrm{t}_{\min }=34.69 \mathrm{~min}$.

Methyl (2R,3R,4R,5S)-5-(4-chlorophenyl)-3,4-bis(phenylsulfonyl)pyrrolidine-2carboxylate 10i: Pale yellow oil; $[\alpha]_{\mathrm{D}}{ }^{20}=-3^{\circ}\left(c 0.5, \mathrm{CH}_{2} \mathrm{Cl}_{2}, 40 \%\right.$ ee $)$; IR (neat) $v_{\max }$ 2957, 1747, 1308, $1249 \mathrm{~cm}^{-1} ;{ }^{1} \mathrm{H}$ NMR $\delta_{\mathrm{H}}: 2.98$ (br. t, $\left.1 \mathrm{H}, \mathrm{NH}\right) .3 .66\left(\mathrm{~s}, 3 \mathrm{H}, \mathrm{CO}_{2} \mathrm{CH}_{3}\right.$ ), 3.78 (s, $\left.3 \mathrm{H}, \mathrm{ArOCH}_{3}\right), 3.83$ (t, $\left.J=6.5 \mathrm{~Hz}, 1 \mathrm{H} \mathrm{CHCO}_{2}\right), 4.33(\mathrm{dd}, J=7.0,2.5 \mathrm{~Hz}, 1 \mathrm{H}$, CHS), 4.42 (dd, $J=6.5,2.5 \mathrm{~Hz}, 1 \mathrm{H}, \mathrm{CHS}), 4.59$ (dd, $J=10.0,7.0 \mathrm{~Hz}, 1 \mathrm{H}, \operatorname{ArCHN}$ ), 6.79-6.75 (m, 2H, ArH), $7.43(\mathrm{t}, J=7.7 \mathrm{~Hz}, 2 \mathrm{H}, \operatorname{Ar} H), 7.53(\mathrm{td}, J=13.5,6.6 \mathrm{~Hz}, 5 \mathrm{H}$ $\operatorname{Ar} H), 7.67(\mathrm{dt}, J=10.5,4.1 \mathrm{~Hz}, 5 \mathrm{H}, \operatorname{Ar} H) ;{ }^{13} \mathrm{C}$ NMR $\delta_{\mathrm{C}}: 52.5,55.2\left(2 \mathrm{xOCH}_{3}\right), 63.2$, 64.1, 67.8 (2xCHS, and $\mathrm{CHCO}_{2}$ ), 71.1 (ArCHN), 114.1, 128.4, 128.5, 128.6, 129.3, $129.4,130.1,134.3,134.5,136.9,138.1,159.5(\mathrm{ArC}), 167.55(\mathrm{CO})$; MS (EI) $m / z(\%)$ : 515 (0.03), 277 (23), 141 (56), 91 (22), 77 (100); HRMS for $\mathrm{C}_{25} \mathrm{H}_{25} \mathrm{NO}_{7} \mathrm{~S}_{2}$ requires 515.5985; found 5155991; HPLC (Chiralpak AD), $n$-hexane $/ i$-PrOH:70/30, $1 \mathrm{~mL} / \mathrm{min}$, $\mathrm{t}_{\text {maj }}=31.3 \mathrm{~min}, \mathrm{t}_{\min }=36.4 \mathrm{~min}$.

Methyl (2S,3R,4S,5R)-4-benzoyl-3,5-diphenylpyrrolidine-2-carboxylate 15a ${ }^{\left[{ }^{[\mathrm{b}]}\right.}$

Methyl (2S,3R,4S,5R)-4-benzoyl-5-(naphth-2-yl)-3-phenylpyrrolidine-2-carboxylate 15b: Colourless solid, mp: $128-129^{\circ} \mathrm{C}$ ( $n$-hexane/ethyl acetate); $[\alpha]_{\mathrm{D}}{ }^{20}=-37(c 0.8$, $\mathrm{CH}_{2} \mathrm{Cl}_{2}, 60 \%$ ee by HPLC). IR (neat) $v_{\max }: 3345,1736,1677 \mathrm{~cm}^{-1} ;{ }^{1} \mathrm{H}$ NMR $\delta_{\mathrm{H}} 3.35$ (br s, $\mathrm{NH}$ ), 3.76 (s, 3H, $\mathrm{CH}_{3}$ ). 4.19 (deform. t, $J=7.5 \mathrm{~Hz}, 1 \mathrm{H}, \mathrm{CHCHCO}$ ) 4.25 (d, $J=8.0$ $\left.\mathrm{Hz}, 1 \mathrm{H}, \mathrm{CHCO}_{2}\right), 4.62(\mathrm{dd}, J=15.0,7.5 \mathrm{~Hz}, 1 \mathrm{H}, \mathrm{CHCOPh}), 5.17(\mathrm{~d}, J=8.5 \mathrm{~Hz}, 1 \mathrm{H}$ $\operatorname{ArCHN}), 7.83-7.10(\mathrm{~m}, 17 \mathrm{H}, \mathrm{Ar} H) ;{ }^{13} \mathrm{C}$ NMR $\delta_{\mathrm{C}}: 52.3,52.7\left(\mathrm{CH}_{3}, \mathrm{CPh}\right), 60.5$ $\left(\mathrm{CHCO}_{2}\right), 66.7,67.8(2 \mathrm{xCHN}), 125.0,125.1,125.8,125.9,126.3,126.4,127.1,127.6$, 127.7, 127.8, 127.9, 128.1, 128.5, 128.8, 132.8, 136.4, 137.4, $140.7(\mathrm{ArC}), 173.3\left(\mathrm{CO}_{2}\right)$ $198.8(\mathrm{COPh})$; MS (EI): $m / z(\%): 435\left(\mathrm{M}^{+}, 10\right)$; HRMS-ESI for $\mathrm{C}_{29} \mathrm{H}_{25} \mathrm{NO}_{3}$ requires 435.5135; found: 435. 5137; HPLC (Chiralcel OD-H), $n$-hexane/i-PrOH: 99/1, 1 $\mathrm{mL} / \mathrm{min}, \mathrm{t}_{\mathrm{maj}}=17.3 \mathrm{~min}, \mathrm{t}_{\mathrm{min}} 25.8 \mathrm{~min}$.

2-Methyl (2S,3R,4R,5S)-4-nitro-3,5-diphenyl-2-pyrrolidinocarboxylate 16a. ${ }^{[7 f]}$

\section{Acknowledgements}

This work has been supported by the DGES of the Spanish Ministerio de Ciencia e Innovación (MICINN) (Consolider INGENIO 2010 CSD2007-00006, FEDERCTQ2007-62771/BQU, CTQ2007/67528, CTQ2010-20387 and by the HispanoBrazilian project PHB2008-0037-PC), Generalitat Valenciana (PROMETEO/ 2009/039), the Basque government (Grant IT-324-07) and by the University of Alicante. M. M.-R. Also thanks DGES for a grant. The authors also thank the SGI/IZOSGIker of UPV/EHU for allocation of computational resources. 
Received: ((will be filled in by the editorial staff))

Revised: ((will be filled in by the editorial staff))

Published online: ((will be filled in by the editorial staff))

Table 2. 1,3-DC of iminoesters 3a and maleimides $\mathbf{4}$ catalyzed by chiral complex $\mathbf{2}(\mathrm{X}=\mathrm{TFA})$.

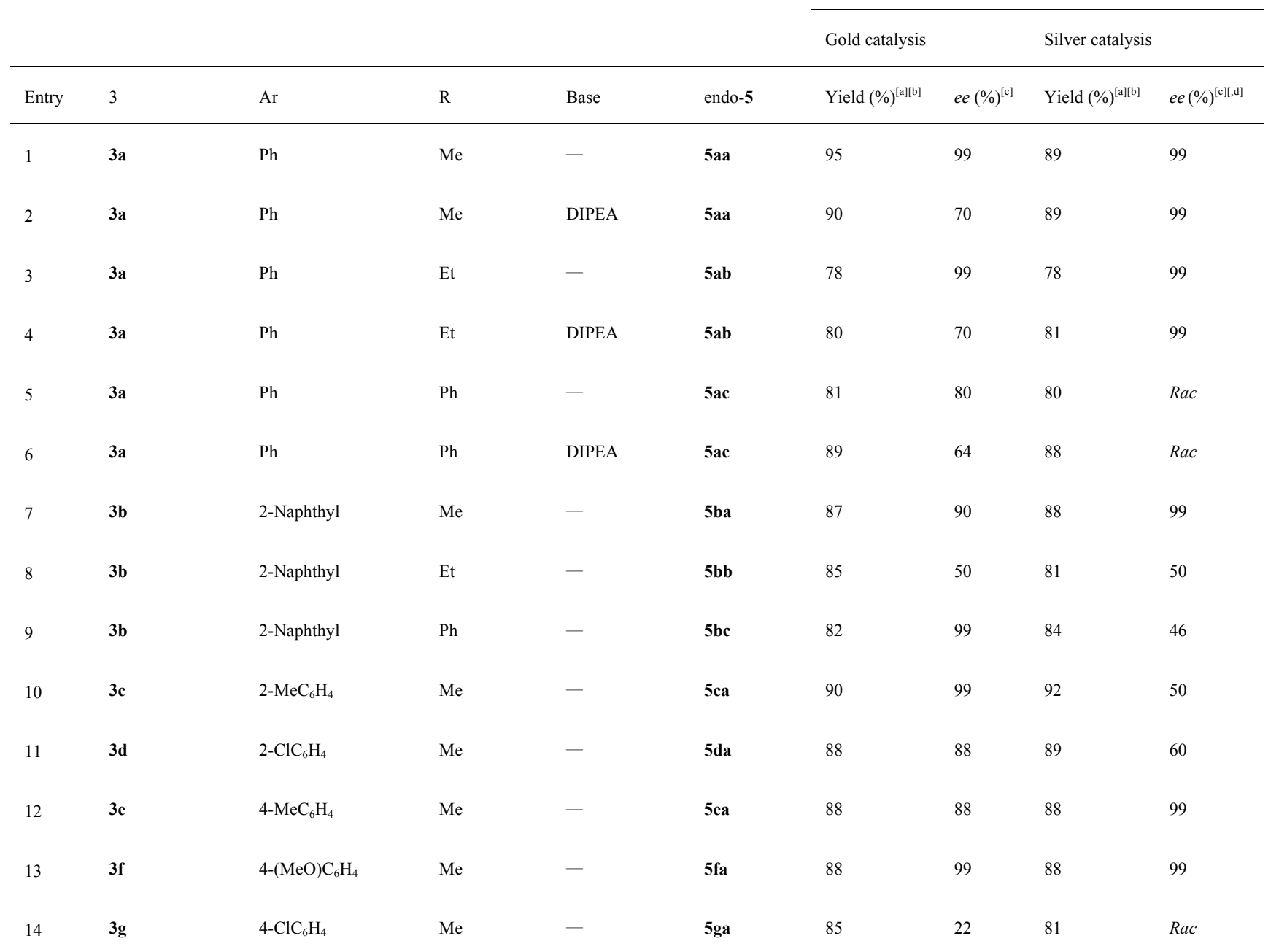

[a] Isolated yields after flash chromatography (silica gel). [b] The observed endo:exo ratio was $>98: 2$ ( ${ }^{1} \mathrm{H}$ NMR). [c] Determined by using analytical chiral HPLC columns.

[d] The reaction was performed exclusively with $\left(S_{\mathrm{a}}\right)$-Binap-AgTFA 6 (10 mol\%). 


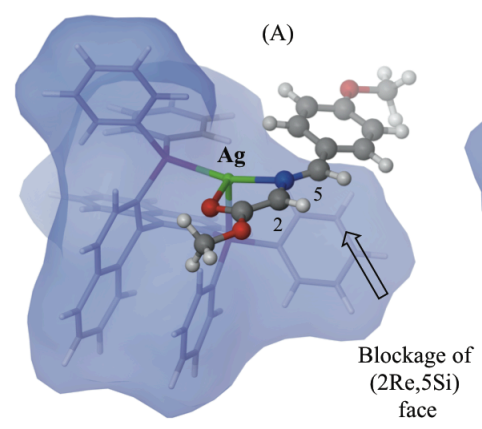

$\left(\mathrm{S}_{\mathrm{a}}\right)$-Binap-Ag-I
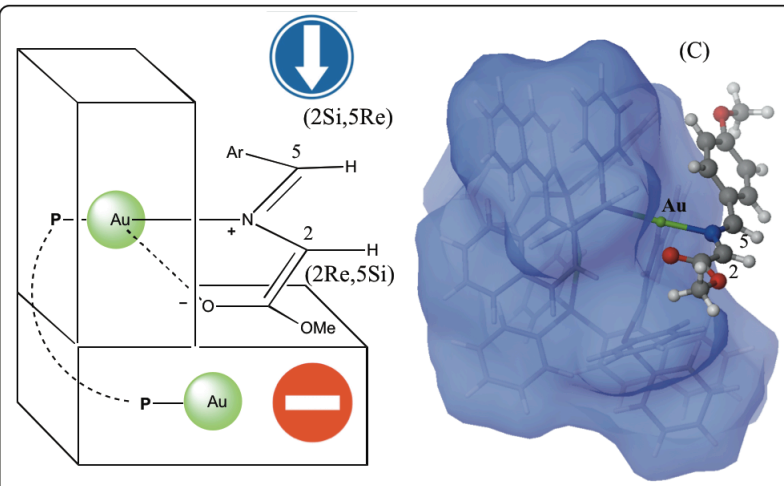

$\left[\left(\mathrm{S}_{\mathrm{a}}\right) \text {-Binap-Au }\right]_{2}-\mathrm{I}$

$(0.0)$

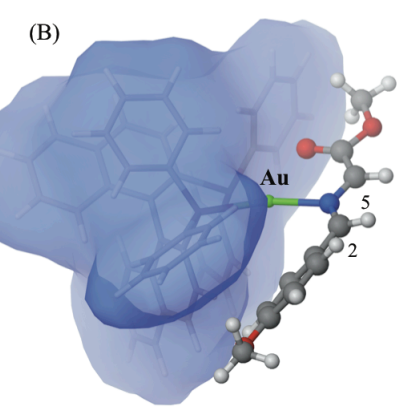

$\left(\mathrm{S}_{\mathrm{a}}\right)$-Binap-Au-I
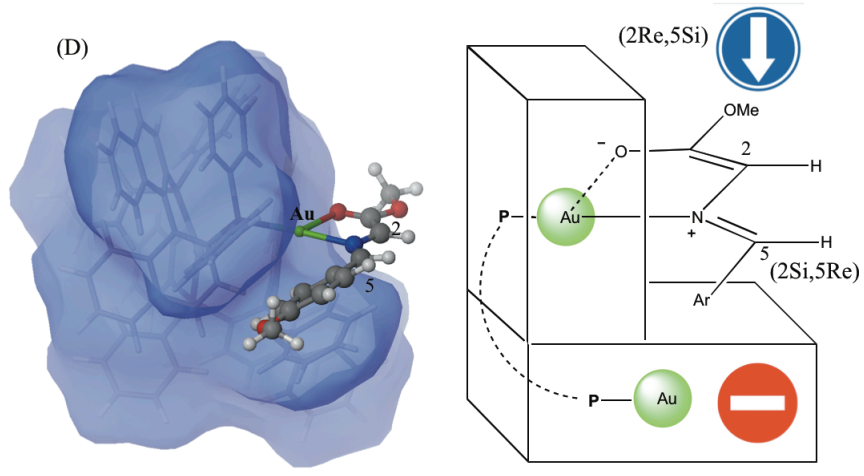

$\left[\left(\mathrm{S}_{\mathrm{a}}\right) \text {-Binap-Au }\right]_{2}-\mathrm{I}-\mathrm{b}$ $(+6.0)$

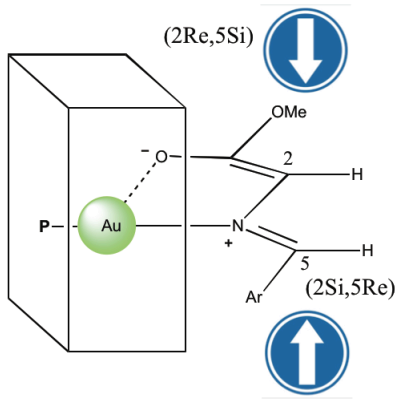

Figure 2. Geometry of the most stable $\left(S_{\mathrm{a}}\right)$-Binap $\mathrm{Ag}(\mathrm{I})$ or $\mathrm{Au}(\mathrm{I})$ complexes computed at ONIOM(B3LYP/LanL2DZ:UFF) level of theory. The B3LYP/LanL2DZ and UFF levels in the ONIOM calculation are depicted in ball \& stick and tube representations respectively. Number in parentheses correspond to the relative Gibbs free energies at $298 \mathrm{~K}$ and are given in $\mathrm{kcal} \mathrm{mol}^{-1}$. Surfaces represent e solvent accessible surface with a probe radius of $1.9 \AA$. For panels (B), (C) and (D) a schematic cartoon of the possible stereochemical course of the corresponding $(3+2)$ cycloaddition is also displayed.

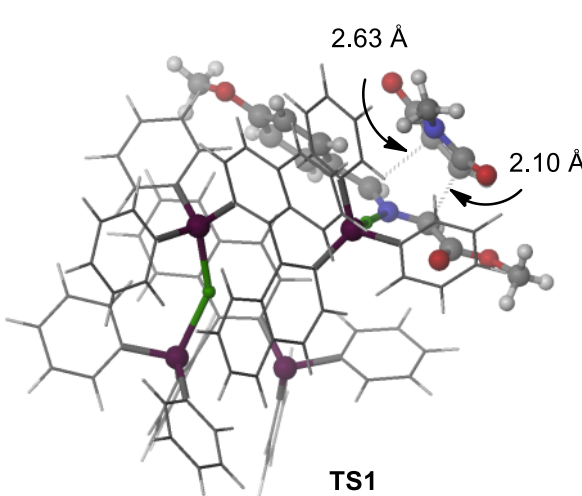

TS1
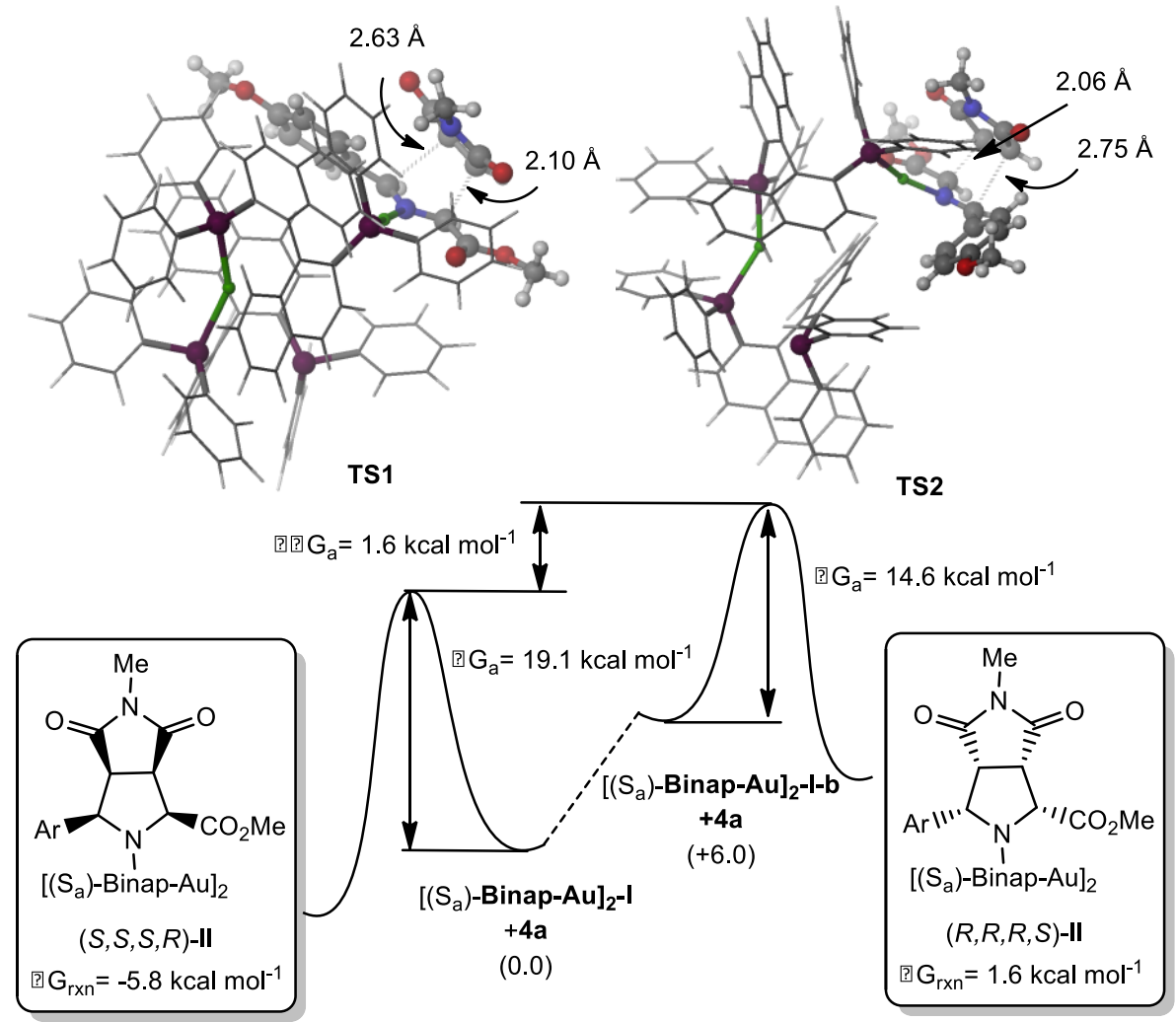

Figure 3. Gibbs activation energy and main geometrical features of the transition structures computed reaction paths corresponding for the two possible 1,3-DC of Au(I) ylide dimeric complexes and $N$-Methyl maleimide computed at ONIOM(B3LYP/LanL2DZ:UFF) level of theory. High level and low-level layers were represented as ball \& stick and wireframe models respectively. 


\section{References}

[1] a) Hashmi, A. S. K. Chem. Rev. 2007, 107, 3180-3211. b) Wiedenhoefer, R. A. Chem. Eur. J. 2008, 14, 5382-5391. c) Gorin, D. J.; Sherry, B. D.; Toste, F. D. Chem. Rev. 2008, 108, 3351-3378. d) Sengupta, S.; Shi, X. Chem CatChem 2010, 2, 609-619.

[2] For recent reviews, see: a) Padwa, A.; Pearson, W. H. In Synthetic Applications of 1,3-Dipolar Cycloaddition Chemistry Towards Heterocycles and Natural Products. John Wiley \& Sons, New Jersey 2003. b) Nájera, C.; Sansano, J. M. Curr. Org. Chem. 2003, 7, 1105-1150. c) Nair, V.; Suja, T. D. Tetrahedron 2007, 63, 12247-12275; d) Padwa, A.; Bur, S. K. Tetrahedron 2007, 63, 5341-5378; e) Pellissier, H. Tetrahedron 2007, 63, 3235-3285.

[3] a) Nájera, C.; Sansano, J. M. Topics in Heterocyclic Chemistry 2008, 12, 117-145. b) Stanley, L. M.; Sibi, M. P. Chem Rev 2008, 108, 2887-2902; c) Álvarez-Corral, M.; Muñoz-Dorado, M.; Rodríguez-García, I. Chem. Rev. 2008, 108, 3174-3198. d) Naodovic, M.; Yamamoto, H. Chem. Rev. 2008, 108, 3132-3148. e) Nájera, C.; Sansano, J. M.; Yus, M. J. Braz. Chem. Soc. 2010, 21, 377-412. f) Kissane, M.; Maguire, A. R. Chem. Soc. Rev. 2010, 39, 845-883.

[4] Allway, P.; Grigg, R. Tetrahedron Lett. 1991, 32, 5817-5820.

[5] Longmire, J. M.; Wang, B.; Zhang, X. J. Am. Chem. Soc. 2002, 124, 13400-13401.

[6] For recent publications, see: a) Nájera, C.; Retamosa, M. G.; Sansano, J. M. Angew. Chem. Int. Ed. 2008, 47, 60556058. b) Nájera, C.; Retamosa, M. G.; Martín-Rodríguez, M.; Sansano, J. M.; de Cózar, A.; Cossío, F. P. Eur. J. Org. Chem. 2009, 5622-5634. c) Yu, S. B.; Hu, X.-P.; Deng, J.; Wang, D.-Y.; Duan, Z.-C.; Zheng, Z. Tetrahedron: Asymmetry 2009, 20, 621-625. d) Wang, C.-J.; Xue, Z.-Y.; Liang, G.; Lu, Z. Chem. Commun. 2009, 2905-2907. e) Martín-Rodríguez, M.; Nájera, C.; Sansano, J. M.; Costa, P. R. R.; de Lima, E. C.; Dias, A. G. Synlett 2010, 962967. f) Robles-Machín, R.; Alonso, I.; Adrio, J.; Carretero, J. C. Chem. Eur. J. 2010, 16, 5286-5291. g) Oura, I.; Shimizu, K.; Ogata, K.; Fukuzawa, S. Org. Lett. 2010, 12, 1752-1755. h) Shimizu, K.; Ogata, K.; Fukuzawa, S. Tetrahedron Lett. 2010, 51, 5068-5070. i) Eröksüz, S.; Dogan, Ö.; Garner, P. P. Tetrahedron: Asymmetry 2010, 21, 2535-2541. j) Xue, Z.-Y.; Liu, T.-L.; Lu, Z.; Huang, H.; Tao, H.-Y.; Wang, C.-J. Chem. Commun. 2010, 46, 17271729. k) Liang, G.; Tong, M.-C.; Wang, C.-J. Adv. Synth. Catal. 2010, 351, 3101-3106.

[7] For recent publications, see: a) López-Pérez, A.; Adrio, J.; Carretero, J. C. J. Am. Chem. Soc. 2008, 130, 1008410085; b) Herández-Toribio, J.; Gómez-Arrayás, R.; Martín-Matute, B.; Carretero, J. C. Org. Lett. 2009, 11, 393396. c) Llamas, T.; Gómez-Arrayás, R.; Carretero, J. C. Org. Lett. 2006, 8, 1795-1798. d) Llamas, T.; GómezArrayás, R.; Carretero, J. C. Synthesis 2007, 950; e) Martín-Matute, B.; Pereira, S. I.; Peña-Cabrera, E.; Adrio, J.; Silva, A. M. S.; Carretero, J. C. Adv. Synth. Catal. 2007, 349, 1714-1724. f) Cabrera, S.; Gómez-Arrayás, R.; Martín-Matute, B.; Cossío, F. P.; Carretero, J. C. Tetrahedron 2007, 63, 6587-6602. g) Shi, M.; Shi, J. W. Tetrahedron: Asymmetry 2007, 18, 645-650. h) Fukuzawa, S.; Oki, H. Org. Lett. 2008, 10, 1747-1750. i) Wang, C. J.; Liang, G.; Xue, Z. Y.; Gao, F. J. Am. Chem. Soc. 2008, 130, 17250-17251. j) López-Pérez, A.; Adrio, J.; Carretero, J. C. Angew. Chem. Int. Ed. 2009, 48, 340-343; k) Hernández-Toribio, J.; Gómez-Arrayás, R.; MartínMatute, B.; Carretero, J. C. Org. Lett. 2009, 11, 393-396. 1) Arai, T.; Mishiro, A.; Yokoyama, N.; Suzuki, K.; Sato, H. J. Am. Chem. Soc. 2010, 132, 5338-5339. m) Zhang, C.; Yu, S. B.; Hu, X. P.; Wang, D. Y.; Zheng, Z. Org. Lett. 2010, 12, 5542-5545. n) Padilla, S.; Tejero, R.; Adrio, J.; Carretero, J. C. Org. Lett. 2010, 12, 5608-5611. o) Arai, T.; Mishiro, A.; Yokoyama, N.; Suzuki, K.; Sato, H. J. Am. Chem. Soc. 2010, 132, 5338-5339. p) Liu, T.-L.; He, Z.L.; Tao, H.-Y.; Cai, Y.-P.; Wang, C.-J. Chem. Commun. 2010, 47, 2616-2618.

[8] a) Nájera, C.; Retamosa, M. G.; Sansano, J. M. Org. Lett. 2007, 9, 4025-4028. b) Nájera, C.; Retamosa, M. G.; Sansano, J. M.; de Cózar, A.; Cossío, F. P. Tetrahedron: Asymmetry 2008, 19, 2913-2923.

[9] a) Melhado, A. D.; Luparia, M.; Toste, F. D. J. Am. Chem. Soc. 2007, 129, 12638-12639. b) Melhado, A. D.; Amarante, G. W.; Wang, Z. J.; Luparia, M.; Toste, F. D. J. Am. Chem. Soc. 2011, 133, 3517-3527.

[10] For a preliminary communication, see: Martín-Rodríguez, M.; Nájera, C.; Sansano, J. M.; Wu, F. L. Tetrahedron: Asymmetry 2010, 21, 1184-1186, and corringendum 2010, 21, 2559.

[11] The reactivity of the dimeric gold complex $\left[\left(S_{\mathrm{a}}\right) \text {-Binap-AuTFA }\right]_{2}$ has been studied and compared with several silver complexes in the elaboration of the key precursor of the $2^{\text {nd }}$ generation GSK-inhibitors of the virus responsible of hepatitis C: submitted to Beilstein J. Org. Chem.

[12] Wheaton, C. A.; Jennings, M. C.; Puddephatt, R. J. Z. Naturforsch 2009, 64b, 1569-1477.

[13] Kagan, H. B. Synlett 2001, 888-899 and references cited therein.

[14] a) Cabrera, S.; Gómez Arrayás, R.; Martín-Matute, B.; Cossío, F. P.; Carretero, J. C. Tetrahedron 2007, 63, 65876602. b) de Cózar, A.; Cossío, F. P. Phys. Chem. Chem. Phys. 2011, 13, 10858-10868.

[15] a) Svensson, M.; Humbel, S.; Morokuma, K. J. Chem. Phys. 1996, 105, 3654-3661. b) Vreven, T.; Morokuma, K. J. Comput. Chem. 2000, 21, 1419-1432. c) Dapprich, S.; Komaromi, I.; Byun, K. S.; Morokuma, K.; Frisch, M. J. J. Mol. Struc. (Theochem) 1999, 461-462.

[16] Frisch M.J. et al. Gaussian09, revision A.1, Gaussian Inc., Wallingford CT, 2009 (full reference in the supporting information).

[17] a) Becke, A. D. J. Chem. Phys. 1983, 98, 5648-5652. b) Becke, A. D. Phys. Rev. A 1998, 38, 3098-3100. c) Kohn, W.; Becke, A. D.; Parr, R. G. J. Phys. Chem. 1996, 100, 12974-12980. d) Lee, C.; Yang, W.; Parr, R. G. Phys. Rev. B 1998, 37, 785-789. e) Vosko, S. H.; Wilk, L.; Nusair, M. Can. J. Phys. 1980, 58, 1200-1211. f) Stephens, P. J.; Devlin, F. J.; Chavalowski, C. F.; Frisch, M. J. J. Phys. Chem. 1994, 98, 11623-11627.

[18] May, P. J.; Wadt, W. R. J. Chem. Phys. 1985, 82, 299-303.

[19] Rappé, A. K.; Casewit, C. J.; Colwell, K. S.; Goddard III, W. A.; Skid, W. M. J. Am. Chem. Soc. 1992, 114, 1002410035.

[20] a) Simón, L.; Goodman, J. M. J. Am. Chem. Soc. 2008, 130, 8741-8747. b) Simón, L.; Goodman, J. M. J. Am. Chem. Soc. 2009, 131, 4070-4077.

[21] Oderaotoshi, Y.; Cheng, W.; Fujitomi, S.; Kasano, Y.; Minakata, S.; Komatsu, M. Org. Lett. 2003, 5, 5043-5046.

[22] Dogan, O.; Koyuncu, H.; Garner, P.; Bulut, A.; Youngs, W. J.; Panzner, M. Org. Lett. 2006, 8, 4687-4690. 
Entry for the Table of Contents (Please choose one layout only)

Layout 1:

Catch Phrase

Author(s), Corresponding

Author $(s) *$............ Page - Page

Title Text

((The TOC Graphic should not exceed the size of this area))

Text for Table of Contents, max. 450 characters.

\section{Layout 2:}

Chiral gold(I)-catalyzed 1,3-DC shows NLE

María Martín-Rodríguez, Carmen Nájera, * José M. Sansano, Abel de Cózar, and Fernando P. Cossio. * ....... Page - Page

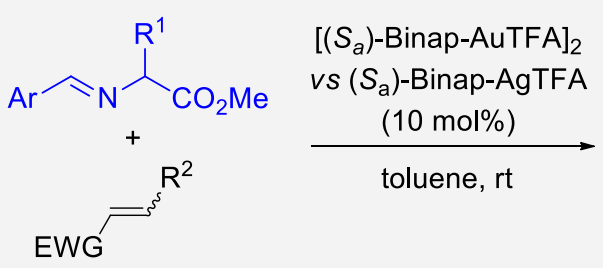

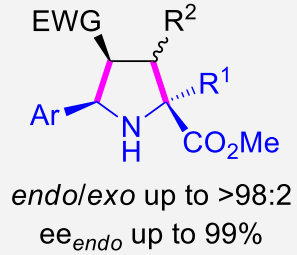

ee $_{\text {endo }}$ up to $99 \%$

The enantioselective 1,3-DC of The origin of the enantioselection, as azomethine ylides and electrophilic well as the observed NLE, are analyzed alkenes has been successfully catalysed using computational tools. by $\left[\left(S_{\mathrm{a}}\right) \text {-Binap-AuTFA }\right]_{2}$ complex.

trifluoroacetate complexes as catalysts in 1,3-dipolar cycloadditions of azomethine ylides 


\section{SUPPORTING INFORMATION}
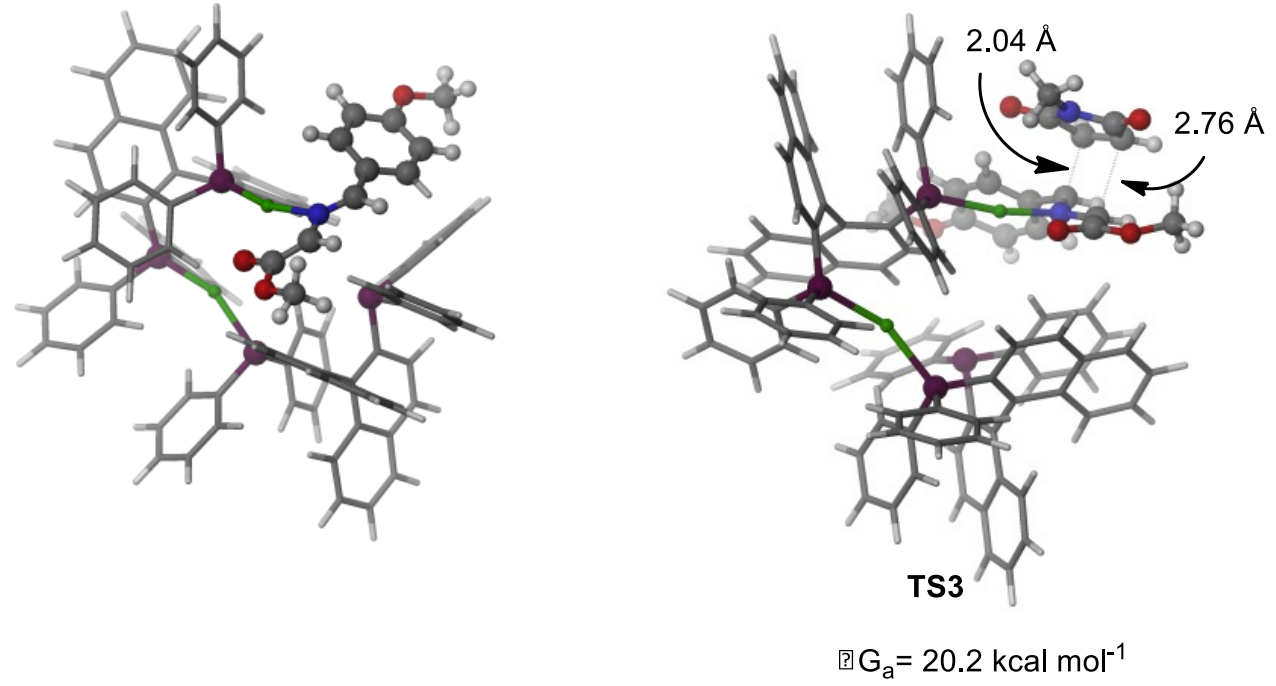

FigureS1. Geometrical features of the most stable ylide heterodimer complex $\left[\left(\mathrm{S}_{\mathrm{a}}\right)^{-}\right.$ Binap, $\left(\mathrm{R}_{\mathrm{a}}\right)$-Binap- $\left.\mathrm{Au}_{2}\right]-\mathrm{I}$ and the TS corresponding to the reaction of that ylide and NMM computed at ONIOM(B3LYP/LanL2DZ:UFF) level of theory. High level and low-level layers were represented as ball \& stick and wireframe models respectively.

Table S1. Total electronic energies ${ }^{\mathrm{a}}$ (E, in a.u.), thermal corrections to Gibbs free energies ${ }^{\mathrm{b}}$ (TCGE, in a.u.) and number of imaginary frequencies ${ }^{\mathrm{c}}$ (NIMAG) of all stationary points discussed in the main text.

\begin{tabular}{cccc}
\hline Structure & $\mathrm{E}$ & TCGE & NIMAG $(v)$ \\
\hline $\mathbf{3}$ & -707.2881858 & 0.181147 & 0 \\
$\mathbf{4 a}$ & -398.6789855 & 0.064225 & 0 \\
$\mathbf{5}$ & -1106.0113219 & 0.275834 & 0 \\
{$\left[\left(\mathrm{~S}_{\mathrm{a}}\right)-\right.$ Binap Ag $]-\mathbf{I}$} & -868.862405 & 0.795801 & 0 \\
{$\left[\left(\mathrm{~S}_{\mathrm{a}}\right)-\right.$ Binap Au $]-\mathbf{I}$} & -858.551914 & 0.796065 & 0 \\
{$\left[\left(\mathrm{~S}_{\mathrm{a}}\right)-\text { Binap Au }\right]_{2}-\mathbf{I}$} & -1010.201625 & 1.430058 & 0 \\
{$\left[\left(\mathrm{~S}_{\mathrm{a}}\right)-\text { Binap Au }\right]_{2}-\mathbf{I}-\mathbf{b}$} & -1010.191870 & 1.429869 & 0 \\
{$\left[\left(\mathrm{~S}_{\mathrm{a}}\right)-\mathrm{Binap}-\left(\mathrm{R}_{\mathrm{a}}\right)-\mathrm{Binap}-\mathrm{Au}_{2}\right]-\mathbf{I}$} & -1010.215705 & 1.418365 & 0 \\
$(S, S, S, R)-\mathbf{I I}$ & -1408.921851 & 1.526251 & 0 \\
$(R, R, R, \mathrm{~S})-\mathbf{I I}$ & -1408.911811 & 1.527335 & 0 \\
$\mathbf{T S 1}$ & -1408.878344 & 1.522479 & $1(-300.875)$ \\
$\mathbf{T S 2}$ & -1408.875365 & 1.521839 & $1(-292.414)$ \\
$\mathbf{T S 3}$ & -1408.89197 & 1.512060 & $1(-342.801)$
\end{tabular}

${ }^{a}$ Computed at level ONIOM(B3LYP/LanL2DZ:UFF) of theory. ${ }^{b}$ Computed at $298 \mathrm{~K}$ at ONIOM(B3LYP/LanL2DZ:UFF) level of theory. ${ }^{\mathrm{c}}$ If $\mathrm{NIMAG}=1$, the imaginary frequency $v$ (in parentheses) is given in $\mathrm{cm}^{-1}$ 
Table S2. Calculated kinetic constants calculated employing Eyring equations and the computed activation free energy barriers.

\begin{tabular}{cc}
\hline & $\mathrm{K}\left(\mathrm{s}^{-1}\right)$ \\
\hline$k_{\mathrm{HOMO}}$ & $6.110^{-2}$ \\
$k_{\mathrm{HET}}$ & $9.610^{-3}$ \\
\hline
\end{tabular}

Cartesian coordinates (optimized at the ONIOM(B3LYP/LanL2DZ:UFF) level) of all the stationary points discussed in the main text.

\begin{tabular}{|c|c|c|c|c|c|}
\hline \multirow{2}{*}{$\begin{array}{l}\text { Center } \\
\text { Number }\end{array}$} & \multirow{2}{*}{$\begin{array}{l}\text { Atomic } \\
\text { Number }\end{array}$} & \multirow{2}{*}{$\begin{array}{c}\text { Atomic } \\
\text { Type }\end{array}$} & \multicolumn{3}{|c|}{ Coordinates (Angstroms) } \\
\hline & & & $\mathrm{x}$ & $\mathrm{Y}$ & $\mathrm{z}$ \\
\hline 1 & 6 & 0 & 0.537234 & 0.893114 & -0.433045 \\
\hline 2 & 6 & 0 & 2.846655 & 0.658035 & -0.954946 \\
\hline 3 & 6 & 0 & 3.720396 & 0.110894 & 0.174168 \\
\hline 4 & 8 & 0 & 3.405569 & 0.046550 & 1.370620 \\
\hline 5 & 6 & 0 & -0.856158 & 0.442350 & -0.288241 \\
\hline 6 & 6 & 0 & -1.240443 & -0.889960 & -0.593911 \\
\hline 7 & 6 & 0 & -1.840999 & 1.344122 & 0.165038 \\
\hline 8 & 6 & 0 & -2.566430 & -1.297937 & -0.449752 \\
\hline 9 & 1 & 0 & -0.477047 & -1.580456 & -0.939479 \\
\hline 10 & 6 & 0 & -3.181828 & 0.947109 & 0.316013 \\
\hline 11 & 1 & 0 & -1.560709 & 2.368255 & 0.406873 \\
\hline 12 & 6 & 0 & -3.543574 & -0.380356 & 0.006174 \\
\hline 13 & 1 & 0 & -2.878535 & -2.312713 & -0.677210 \\
\hline 14 & 1 & 0 & -3.917679 & 1.662542 & 0.668705 \\
\hline 15 & 7 & 0 & 1.484681 & 0.119065 & -0.860462 \\
\hline 16 & 8 & 0 & -4.835130 & -0.890646 & 0.115478 \\
\hline 17 & 8 & 0 & 4.955327 & -0.279192 & -0.306295 \\
\hline 18 & 6 & 0 & -5.900142 & -0.013933 & 0.584987 \\
\hline 19 & 1 & 0 & -6.800464 & -0.631212 & 0.587155 \\
\hline 20 & 1 & 0 & -5.696668 & 0.348109 & 1.602238 \\
\hline 21 & 1 & 0 & -6.038404 & 0.840506 & -0.091891 \\
\hline 22 & 6 & 0 & 5.923325 & -0.791334 & 0.682408 \\
\hline 23 & 1 & 0 & 6.130966 & -0.028007 & 1.439123 \\
\hline 24 & 1 & 0 & 5.521915 & -1.684920 & 1.170232 \\
\hline 25 & 1 & 0 & 6.817823 & -1.026519 & 0.104307 \\
\hline 26 & 1 & 0 & 3.292656 & 0.358619 & -1.907317 \\
\hline 27 & 1 & 0 & 0.729482 & 1.943886 & -0.153644 \\
\hline 28 & 1 & 0 & 2.889556 & 1.762130 & -0.877653 \\
\hline
\end{tabular}

$4 a$

\begin{tabular}{|c|c|c|c|c|c|}
\hline \multirow{2}{*}{$\begin{array}{l}\text { Center } \\
\text { Number }\end{array}$} & \multirow{2}{*}{$\begin{array}{l}\text { Atomic } \\
\text { Number }\end{array}$} & \multirow{2}{*}{$\begin{array}{c}\text { Atomic } \\
\text { Type }\end{array}$} & \multicolumn{3}{|c|}{ Coordinates (Angstroms) } \\
\hline & & & $\mathrm{x}$ & $\mathrm{Y}$ & $\mathrm{Z}$ \\
\hline & & & & 列 & 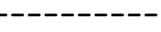 \\
\hline 1 & 6 & 0 & -0.682413 & -1.636126 & 0.000024 \\
\hline 2 & 6 & 0 & 0.669064 & -1.641522 & 0.000016 \\
\hline 3 & 1 & 0 & 1.353465 & -2.479251 & -0.000015 \\
\hline 4 & 1 & 0 & -1.373952 & -2.467929 & -0.000017 \\
\hline 5 & 7 & 0 & 0.003488 & 0.596717 & -0.000056 \\
\hline 6 & 6 & 0 & 0.010785 & 2.061590 & 0.000027 \\
\hline 7 & 1 & 0 & 0.522033 & 2.443482 & -0.889946 \\
\hline 8 & 1 & 0 & -1.028200 & 2.402197 & -0.000372 \\
\hline 9 & 1 & 0 & 0.521354 & 2.443394 & 0.890419 \\
\hline 10 & 6 & 0 & 1.157632 & -0.213904 & -0.000017 \\
\hline 11 & 8 & 0 & 2.331923 & 0.197276 & 0.000005 \\
\hline 12 & 6 & 0 & -1.159813 & -0.205206 & -0.000003 \\
\hline 13 & 8 & 0 & -2.330753 & 0.214235 & 0.000000 \\
\hline
\end{tabular}

5

Center Atomic Atomic Coordinates (Angstroms)




\begin{tabular}{|c|c|c|c|c|c|}
\hline Number & Number & Type & $\mathrm{x}$ & $\mathrm{Y}$ & $\mathrm{Z}$ \\
\hline 1 & 6 & 0 & 0.141184 & -0.796526 & 1.160526 \\
\hline 2 & 6 & 0 & -2.185796 & -1.077005 & 0.734155 \\
\hline 3 & 6 & 0 & -3.281259 & -1.397757 & -0.274713 \\
\hline 4 & 8 & 0 & -3.115675 & -1.910592 & -1.389327 \\
\hline 5 & 6 & 0 & 1.530470 & -0.717383 & 0.549767 \\
\hline 6 & 6 & 0 & 1.710272 & -0.517615 & -0.839036 \\
\hline 7 & 6 & 0 & 2.670958 & -0.835084 & 1.362705 \\
\hline 8 & 6 & 0 & 2.992847 & -0.431954 & -1.391273 \\
\hline 9 & 1 & 0 & 0.836349 & -0.445912 & -1.480530 \\
\hline 10 & 6 & 0 & 3.968762 & -0.742407 & 0.826025 \\
\hline 11 & 1 & 0 & 2.555286 & -0.995183 & 2.433707 \\
\hline 12 & 6 & 0 & 4.127948 & -0.537085 & -0.559338 \\
\hline 13 & 1 & 0 & 3.142766 & -0.283001 & -2.456393 \\
\hline 14 & 1 & 0 & 4.827549 & -0.831294 & 1.483743 \\
\hline 15 & 7 & 0 & -0.848161 & -1.374628 & 0.237511 \\
\hline 16 & 8 & 0 & 5.360969 & -0.432785 & -1.207274 \\
\hline 17 & 8 & 0 & -4.511170 & -1.089879 & 0.260821 \\
\hline 18 & 6 & 0 & 6.576371 & -0.493622 & -0.408083 \\
\hline 19 & 1 & 0 & 7.395392 & -0.368924 & -1.119254 \\
\hline 20 & 1 & 0 & 6.604666 & 0.315638 & 0.334876 \\
\hline 21 & 1 & 0 & 6.672589 & -1.464160 & 0.098887 \\
\hline 22 & 6 & 0 & -5.672811 & -1.181099 & -0.642675 \\
\hline 23 & 1 & 0 & -5.553451 & -0.454661 & -1.451826 \\
\hline 24 & 1 & 0 & -5.754429 & -2.191153 & -1.056045 \\
\hline 25 & 1 & 0 & -6.534656 & -0.937968 & -0.019879 \\
\hline 26 & 1 & 0 & 0.215075 & -1.371933 & 2.104632 \\
\hline 27 & 1 & 0 & -2.452101 & -1.619208 & 1.664385 \\
\hline 28 & 6 & 0 & -2.040329 & 0.426398 & 1.105407 \\
\hline 29 & 6 & 0 & -0.561907 & 0.578119 & 1.568520 \\
\hline 30 & 1 & 0 & -2.779227 & 0.727203 & 1.853696 \\
\hline 31 & 1 & 0 & -0.441707 & 0.749326 & 2.641091 \\
\hline 32 & 7 & 0 & -0.989952 & 2.142709 & -0.169817 \\
\hline 33 & 6 & 0 & -0.791056 & 3.211393 & -1.154205 \\
\hline 34 & 1 & 0 & 0.137760 & 3.730117 & -0.904191 \\
\hline 35 & 1 & 0 & -0.721845 & 2.789800 & -2.162574 \\
\hline 36 & 1 & 0 & -1.633342 & 3.909890 & -1.125560 \\
\hline 37 & 6 & 0 & -0.043564 & 1.803964 & 0.819581 \\
\hline 38 & 8 & 0 & 1.008998 & 2.430357 & 1.025775 \\
\hline 39 & 6 & 0 & -2.151902 & 1.364341 & -0.107509 \\
\hline 40 & 8 & 0 & -3.108397 & 1.467884 & -0.897595 \\
\hline 41 & 1 & 0 & -0.668810 & -2.295082 & -0.150958 \\
\hline
\end{tabular}

\section{[( $\left.\mathrm{S}_{\mathrm{a}}\right)$-Binap Ag]-I}

\begin{tabular}{|c|c|c|c|c|c|}
\hline \multirow{2}{*}{$\begin{array}{l}\text { Center } \\
\text { Number }\end{array}$} & \multirow{2}{*}{$\begin{array}{l}\text { Atomic } \\
\text { Number }\end{array}$} & \multirow{2}{*}{$\begin{array}{c}\text { Atomic } \\
\text { Type }\end{array}$} & \multicolumn{3}{|c|}{ Coordinates (Angstroms) } \\
\hline & & & $\mathrm{x}$ & $\mathrm{Y}$ & $\mathrm{z}$ \\
\hline--1 & & & & 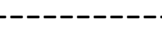 & -- \\
\hline 1 & 47 & 10471001 & 1.125667 & 1.459323 & 1.250834 \\
\hline 2 & 6 & 10061000 & -0.739450 & -1.593572 & 0.920844 \\
\hline 3 & 6 & 10061002 & 0.167026 & -2.688173 & 1.357491 \\
\hline 4 & 6 & 10061002 & 0.416350 & -3.751212 & 0.583626 \\
\hline 5 & 6 & 10061000 & -0.206593 & -3.870390 & -0.743391 \\
\hline 6 & 6 & 10061002 & 0.069743 & -5.039846 & -1.606638 \\
\hline 7 & 6 & 10061000 & -0.491174 & -5.124786 & -2.824331 \\
\hline 8 & 6 & 10061000 & -1.343873 & -4.122714 & -3.307813 \\
\hline 9 & 6 & 10061002 & -1.634783 & -3.032650 & -2.576406 \\
\hline 10 & 6 & 10061000 & -1.051268 & -2.860096 & -1.217286 \\
\hline 11 & 6 & 10061000 & -1.298597 & -1.647577 & -0.382468 \\
\hline 12 & 6 & 10061002 & -2.176320 & -0.580185 & -0.940564 \\
\hline 13 & 6 & 10061002 & -1.676621 & 0.545928 & -1.528235 \\
\hline 14 & 6 & 10061002 & -2.619900 & 1.469269 & -2.228950 \\
\hline 15 & 6 & 10061000 & -3.939711 & 1.234136 & -2.252229 \\
\hline 16 & 6 & 10061000 & -4.480571 & 0.100357 & -1.644291 \\
\hline 17 & 6 & 10061000 & -3.647630 & -0.830807 & -1.007720 \\
\hline 18 & 6 & 10061002 & -4.282417 & -2.034227 & -0.405811 \\
\hline 19 & 6 & 10061000 & -5.614956 & -2.199733 & -0.467276 \\
\hline 20 & 6 & 10061000 & -6.442951 & -1.260020 & -1.097507 \\
\hline 21 & 6 & 10061002 & -5.940008 & -0.153420 & -1.670865 \\
\hline 22 & 6 & 10061000 & -4.866264 & 0.438665 & 2.027355 \\
\hline 23 & 6 & 10061000 & -4.998749 & 1.756863 & 1.583515 \\
\hline 24 & 6 & 10061000 & -3.860953 & 2.508232 & 1.278927 \\
\hline 25 & 6 & 10061000 & -2.590909 & 1.941304 & 1.416803 \\
\hline 26 & 6 & 10061000 & -2.450690 & 0.614805 & 1.847163 \\
\hline 27 & 6 & 10061000 & -3.596695 & -0.133105 & 2.159106 \\
\hline
\end{tabular}




\begin{tabular}{|c|c|c|c|c|c|}
\hline 28 & 15 & 10151003 & -0.763301 & -0.085285 & 1.981290 \\
\hline 29 & 6 & 10061000 & -0.736240 & -0.707361 & 3.708177 \\
\hline 30 & 6 & 10061000 & -0.020363 & 0.019333 & 4.668778 \\
\hline 31 & 6 & 10061000 & 0.025482 & -0.419069 & 5.995631 \\
\hline 32 & 6 & 10061000 & -0.639817 & -1.589711 & 6.369224 \\
\hline 33 & 6 & 10061000 & -1.347658 & -2.325316 & 5.415000 \\
\hline 34 & 6 & 10061000 & -1.395956 & -1.888720 & 4.087632 \\
\hline 35 & 15 & 10151003 & 0.133461 & 0.947502 & -1.441151 \\
\hline 36 & 6 & 10061000 & 0.820246 & -0.362720 & -2.523195 \\
\hline 37 & 6 & 10061000 & 1.818056 & -1.207528 & -2.023822 \\
\hline 38 & 6 & 10061000 & 2.315691 & -2.250860 & -2.809666 \\
\hline 39 & 6 & 10061000 & 1.816028 & -2.454318 & -4.099027 \\
\hline 40 & 6 & 10061000 & 0.812634 & -1.619316 & -4.599908 \\
\hline 41 & 6 & 10061000 & 0.307241 & -0.581132 & -3.811224 \\
\hline 42 & 6 & 10061000 & 0.347036 & 2.512727 & -2.386740 \\
\hline 43 & 6 & 10061000 & 1.161306 & 2.592180 & -3.530682 \\
\hline 44 & 6 & 10061000 & 1.359667 & 3.819613 & -4.170833 \\
\hline 45 & 6 & 10061000 & 0.771961 & 4.979911 & -3.661751 \\
\hline 46 & 6 & 10061000 & -0.005754 & 4.916866 & -2.503973 \\
\hline 47 & 6 & 10061000 & -0.205707 & 3.691996 & -1.861807 \\
\hline 48 & 1 & 10011000 & 2.184562 & -1.075892 & -1.016403 \\
\hline 49 & 1 & 10011000 & 3.078256 & -2.909334 & -2.415028 \\
\hline 50 & 1 & 10011000 & 2.197465 & -3.265673 & -4.705066 \\
\hline 51 & 1 & 10011000 & 0.416973 & -1.785281 & -5.593350 \\
\hline 52 & 1 & 10011000 & -0.482719 & 0.048576 & -4.200062 \\
\hline 53 & 1 & 10011000 & -0.798451 & 3.660207 & -0.956584 \\
\hline 54 & 1 & 10011000 & -0.448027 & 5.818129 & -2.099885 \\
\hline 55 & 1 & 10011000 & 0.930612 & 5.929369 & -4.156417 \\
\hline 56 & 1 & 10011000 & 1.982009 & 3.873392 & -5.054760 \\
\hline 57 & 1 & 10011000 & 1.668490 & 1.723551 & -3.921883 \\
\hline 58 & 1 & 10011000 & 0.499212 & 0.927616 & 4.389967 \\
\hline 59 & 1 & 10011000 & 0.577127 & 0.148415 & 6.733912 \\
\hline 60 & 1 & 10011000 & -0.602704 & -1.929232 & 7.396247 \\
\hline 61 & 1 & 10011000 & -1.854112 & -3.237538 & 5.702702 \\
\hline 62 & 1 & 10011000 & -1.925011 & -2.487426 & 3.359709 \\
\hline 63 & 1 & 10011000 & -2.256501 & 2.340281 & -2.755410 \\
\hline 64 & 1 & 10011000 & -4.590337 & 1.932782 & -2.764443 \\
\hline 65 & 1 & 10011000 & -6.609677 & 0.553755 & -2.151591 \\
\hline 66 & 1 & 10011000 & -7.511575 & -1.431586 & -1.122498 \\
\hline 67 & 1 & 10011000 & -6.061370 & -3.077165 & -0.016866 \\
\hline 68 & 1 & 10011000 & -3.681509 & -2.781399 & 0.102116 \\
\hline 69 & 1 & 10011000 & -2.286682 & -2.277302 & -3.001597 \\
\hline 70 & 1 & 10011000 & -1.771168 & -4.226647 & -4.297061 \\
\hline 71 & 1 & 10011000 & -0.278845 & -5.983708 & -3.448097 \\
\hline 72 & 1 & 10011000 & 0.729919 & -5.829316 & -1.260188 \\
\hline 73 & 1 & 10011000 & 1.093552 & -4.523764 & 0.935688 \\
\hline 74 & 1 & 10011000 & 0.663690 & -2.624913 & 2.318990 \\
\hline 75 & 1 & 10011000 & -5.982353 & 2.193913 & 1.471261 \\
\hline 76 & 1 & 10011000 & -5.748293 & -0.146525 & 2.252874 \\
\hline 77 & 1 & 10011000 & -3.521027 & -1.163034 & 2.467609 \\
\hline 78 & 1 & 10011000 & -1.716203 & 2.530837 & 1.174020 \\
\hline 79 & 1 & 10011000 & -3.962719 & 3.528431 & 0.932503 \\
\hline 80 & 6 & 10061000 & 4.255909 & 1.423589 & 0.306231 \\
\hline 81 & 1 & 10011000 & 5.099077 & 1.869258 & -0.235267 \\
\hline 82 & 6 & 10061000 & 3.227918 & 3.558632 & 0.501765 \\
\hline 83 & 6 & 10061000 & 2.070811 & 4.291324 & 0.838518 \\
\hline 84 & 8 & 10081000 & 0.955803 & 3.829250 & 1.301245 \\
\hline 85 & 6 & 10061000 & 4.303562 & -0.023750 & 0.515504 \\
\hline 86 & 6 & 10061000 & 3.605942 & -0.683943 & 1.567810 \\
\hline 87 & 6 & 10061000 & 5.065450 & -0.833734 & -0.364071 \\
\hline 88 & 6 & 10061000 & 3.622241 & -2.077130 & 1.689207 \\
\hline 89 & 1 & 10011000 & 3.081984 & -0.086848 & 2.310100 \\
\hline 90 & 6 & 10061000 & 5.079359 & -2.235900 & -0.260227 \\
\hline 91 & 1 & 10011000 & 5.629369 & -0.357771 & -1.164536 \\
\hline 92 & 6 & 10061000 & 4.333979 & -2.863632 & 0.758794 \\
\hline 93 & 1 & 10011000 & 3.111128 & -2.581357 & 2.504390 \\
\hline 94 & 1 & 10011000 & 5.656949 & -2.817050 & -0.972812 \\
\hline 95 & 7 & 10071000 & 3.247672 & 2.195108 & 0.680654 \\
\hline 96 & 1 & 10011000 & 4.093120 & 4.086190 & 0.106824 \\
\hline 97 & 8 & 10081003 & 4.245772 & -4.250696 & 0.944410 \\
\hline 98 & 8 & 10081003 & 2.197196 & 5.670007 & 0.611486 \\
\hline 99 & 6 & 10061003 & 4.903902 & -5.117618 & -0.019551 \\
\hline 100 & 1 & 10011000 & 4.674441 & -6.136023 & 0.301396 \\
\hline 101 & 1 & 10011000 & 4.514008 & -4.952583 & -1.034580 \\
\hline 102 & 1 & 10011000 & 5.992923 & -4.967714 & -0.016939 \\
\hline 103 & 6 & 10061003 & 1.044226 & 6.496519 & 0.969480 \\
\hline 104 & 1 & 10011000 & 1.304967 & 7.504212 & 0.636836 \\
\hline 105 & 1 & 10011000 & 0.136279 & 6.142904 & 0.468690 \\
\hline 106 & 1 & 10011000 & 0.875390 & 6.479671 & 2.052566 \\
\hline
\end{tabular}


[( $\left.\mathrm{S}_{\mathrm{a}}\right)$-Binap Au]-I

\begin{tabular}{|c|c|c|c|c|c|}
\hline \multirow{2}{*}{$\begin{array}{l}\text { Center } \\
\text { Number }\end{array}$} & \multirow{2}{*}{$\begin{array}{l}\text { Atomic } \\
\text { Number }\end{array}$} & \multirow{2}{*}{$\begin{array}{c}\text { Atomic } \\
\text { Type }\end{array}$} & \multicolumn{3}{|c|}{ Coordinates (Angstroms) } \\
\hline & & & $\mathrm{x}$ & $\mathrm{Y}$ & $\mathrm{z}$ \\
\hline & & ---- & ------- & 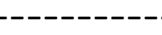 & -ー-ー-ーーーーー \\
\hline 1 & 79 & 10791004 & 1.655741 & 1.428819 & 0.135546 \\
\hline 2 & 6 & 10061000 & -0.017751 & -1.619508 & -0.563837 \\
\hline 3 & 6 & 10061002 & 0.779623 & -1.982376 & -1.762660 \\
\hline 4 & 6 & 10061002 & 0.230457 & -2.598876 & -2.816697 \\
\hline 5 & 6 & 10061000 & -1.203827 & -2.929950 & -2.824259 \\
\hline 6 & 6 & 10061002 & -1.823394 & -3.590526 & -3.994699 \\
\hline 7 & 6 & 10061000 & -3.140892 & -3.854028 & -4.000053 \\
\hline 8 & 6 & 10061000 & -3.958018 & -3.518298 & -2.911681 \\
\hline 9 & 6 & 10061002 & -3.459814 & -2.919189 & -1.816093 \\
\hline 10 & 6 & 10061000 & -2.009056 & -2.591962 & -1.730692 \\
\hline 11 & 6 & 10061000 & -1.410737 & -1.884709 & -0.561145 \\
\hline 12 & 6 & 10061002 & -2.323501 & -1.422514 & 0.514795 \\
\hline 13 & 6 & 10061002 & -2.811835 & -0.151664 & 0.531595 \\
\hline 14 & 6 & 10061002 & -3.863695 & 0.196686 & 1.538554 \\
\hline 15 & 6 & 10061000 & -4.308911 & -0.707046 & 2.424227 \\
\hline 16 & 6 & 10061000 & -3.827723 & -2.017463 & 2.421325 \\
\hline 17 & 6 & 10061000 & -2.868051 & -2.419524 & 1.481934 \\
\hline 18 & 6 & 10061002 & -2.422595 & -3.838102 & 1.502988 \\
\hline 19 & 6 & 10061000 & -2.922434 & -4.690532 & 2.414330 \\
\hline 20 & 6 & 10061000 & -3.873914 & -4.279953 & 3.359286 \\
\hline 21 & 6 & 10061002 & -4.330816 & -3.016186 & 3.393175 \\
\hline 22 & 6 & 10061000 & -0.840511 & -2.069886 & 4.260131 \\
\hline 23 & 6 & 10061000 & -1.318659 & -0.883067 & 4.822445 \\
\hline 24 & 6 & 10061000 & -1.108729 & 0.333777 & 4.167786 \\
\hline 25 & 6 & 10061000 & -0.429032 & 0.362493 & 2.946708 \\
\hline 26 & 6 & 10061000 & 0.017926 & -0.827061 & 2.359702 \\
\hline 27 & 6 & 10061000 & -0.168951 & -2.043250 & 3.033674 \\
\hline 28 & 15 & 10151003 & 0.909110 & -0.743539 & 0.768419 \\
\hline 29 & 6 & 10061000 & 2.387445 & -1.778601 & 1.069701 \\
\hline 30 & 6 & 10061000 & 3.585981 & -1.155985 & 1.442596 \\
\hline 31 & 6 & 10061000 & 4.740364 & -1.917058 & 1.648960 \\
\hline 32 & 6 & 10061000 & 4.704336 & -3.303430 & 1.477992 \\
\hline 33 & 6 & 10061000 & 3.511006 & -3.931014 & 1.109296 \\
\hline 34 & 6 & 10061000 & 2.353655 & -3.172829 & 0.906766 \\
\hline 35 & 15 & 10151003 & -2.215377 & 1.084781 & -0.737241 \\
\hline 36 & 6 & 10061000 & -3.401138 & 0.568391 & -2.054277 \\
\hline 37 & 6 & 10061000 & -2.895353 & 0.257132 & -3.323425 \\
\hline 38 & 6 & 10061000 & -3.752676 & -0.181420 & -4.336493 \\
\hline 39 & 6 & 10061000 & -5.121868 & -0.308358 & -4.087997 \\
\hline 40 & 6 & 10061000 & -5.635147 & 0.005727 & -2.826623 \\
\hline 41 & 6 & 10061000 & -4.779970 & 0.442998 & -1.810895 \\
\hline 42 & 6 & 10061000 & -3.006900 & 2.658711 & -0.172671 \\
\hline 43 & 6 & 10061000 & -3.779462 & 3.446908 & -1.045542 \\
\hline 44 & 6 & 10061000 & -4.325464 & 4.657872 & -0.608765 \\
\hline 45 & 6 & 10061000 & -4.083762 & 5.107828 & 0.690941 \\
\hline 46 & 6 & 10061000 & -3.277711 & 4.357407 & 1.548937 \\
\hline 47 & 6 & 10061000 & -2.722225 & 3.152168 & 1.111214 \\
\hline 48 & 1 & 10011000 & -1.834000 & 0.337810 & -3.521930 \\
\hline 49 & 1 & 10011000 & -3.354254 & -0.430862 & -5.311284 \\
\hline 50 & 1 & 10011000 & -5.784255 & -0.652098 & -4.871719 \\
\hline 51 & 1 & 10011000 & -6.695786 & -0.091802 & -2.635188 \\
\hline 52 & 1 & 10011000 & -5.194663 & 0.686236 & -0.843071 \\
\hline 53 & 1 & 10011000 & -2.089969 & 2.591026 & 1.783110 \\
\hline 54 & 1 & 10011000 & -3.072094 & 4.715590 & 2.549322 \\
\hline 55 & 1 & 10011000 & -4.505976 & 6.045903 & 1.027096 \\
\hline 56 & 1 & 10011000 & -4.928449 & 5.252853 & -1.282390 \\
\hline 57 & 1 & 10011000 & -3.952178 & 3.142299 & -2.068093 \\
\hline 58 & 1 & 10011000 & 3.628039 & -0.081118 & 1.565859 \\
\hline 59 & 1 & 10011000 & 5.665287 & -1.430852 & 1.930870 \\
\hline 60 & 1 & 10011000 & 5.600680 & -3.890655 & 1.629868 \\
\hline 61 & 1 & 10011000 & 3.483392 & -5.004909 & 0.977796 \\
\hline 62 & 1 & 10011000 & 1.438868 & -3.673173 & 0.617282 \\
\hline 63 & 1 & 10011000 & -4.325622 & 1.173717 & 1.548840 \\
\hline 64 & 1 & 10011000 & -5.071385 & -0.415374 & 3.136484 \\
\hline 65 & 1 & 10011000 & -5.068991 & -2.731860 & 4.137402 \\
\hline 66 & 1 & 10011000 & -4.248805 & -4.998901 & 4.076634 \\
\hline 67 & 1 & 10011000 & -2.581386 & -5.717981 & 2.422068 \\
\hline 68 & 1 & 10011000 & -1.680500 & -4.194759 & 0.796021 \\
\hline 69 & 1 & 10011000 & -4.135944 & -2.666423 & -1.006717 \\
\hline 70 & 1 & 10011000 & -5.016425 & -3.741135 & -2.958969 \\
\hline 71 & 1 & 10011000 & -3.583259 & -4.332863 & -4.864339 \\
\hline
\end{tabular}




\begin{tabular}{|c|c|c|c|c|c|}
\hline 72 & 1 & 10011000 & -1.216357 & -3.858235 & -4.854244 \\
\hline 73 & 1 & 10011000 & 0.848744 & -2.849027 & -3.673593 \\
\hline 74 & 1 & 10011000 & 1.836952 & -1.745339 & -1.791240 \\
\hline 75 & 1 & 10011000 & -1.846739 & -0.905963 & 5.766730 \\
\hline 76 & 1 & 10011000 & -1.000634 & -3.012001 & 4.768107 \\
\hline 77 & 1 & 10011000 & 0.181549 & -2.971228 & 2.606613 \\
\hline 78 & 1 & 10011000 & -0.250580 & 1.309517 & 2.455789 \\
\hline 79 & 1 & 10011000 & -1.468477 & 1.254668 & 4.607898 \\
\hline 80 & 6 & 10061000 & 4.282048 & 2.731950 & -0.725024 \\
\hline 81 & 1 & 10011000 & 4.981619 & 3.572206 & -0.743136 \\
\hline 82 & 6 & 10061000 & 2.533192 & 4.289436 & -0.319253 \\
\hline 83 & 6 & 10061000 & 1.204307 & 4.521921 & 0.098751 \\
\hline 84 & 8 & 10081000 & 0.330683 & 3.644354 & 0.435964 \\
\hline 85 & 6 & 10061000 & 4.842630 & 1.405841 & -0.993328 \\
\hline 86 & 6 & 10061000 & 4.145574 & 0.382542 & -1.698952 \\
\hline 87 & 6 & 10061000 & 6.165637 & 1.117772 & -0.574545 \\
\hline 88 & 6 & 10061000 & 4.723118 & -0.873910 & -1.915001 \\
\hline 89 & 1 & 10011000 & 3.163522 & 0.596174 & -2.111192 \\
\hline 90 & 6 & 10061000 & 6.753698 & -0.142548 & -0.777594 \\
\hline 91 & 1 & 10011000 & 6.732476 & 1.886366 & -0.052473 \\
\hline 92 & 6 & 10061000 & 6.020537 & -1.151313 & -1.436160 \\
\hline 93 & 1 & 10011000 & 4.203096 & -1.650291 & -2.468709 \\
\hline 94 & 1 & 10011000 & 7.761748 & -0.324650 & -0.417938 \\
\hline 95 & 7 & 10071000 & 3.009046 & 3.004822 & -0.440020 \\
\hline 96 & 1 & 10011000 & 3.176215 & 5.130419 & -0.557440 \\
\hline 97 & 8 & 10081003 & 6.487721 & -2.450082 & -1.672785 \\
\hline 98 & 8 & 10081003 & 0.879749 & 5.888146 & 0.118128 \\
\hline 99 & 6 & 10061003 & 7.796469 & -2.818727 & -1.155617 \\
\hline 100 & 1 & 10011000 & 7.931301 & -3.867383 & -1.428866 \\
\hline 101 & 1 & 10011000 & 8.592739 & -2.213859 & -1.611927 \\
\hline 102 & 1 & 10011000 & 7.835864 & -2.712834 & -0.061897 \\
\hline 103 & 6 & 10061003 & -0.483706 & 6.227788 & 0.521940 \\
\hline 104 & 1 & 10011000 & -0.517969 & 7.319805 & 0.514037 \\
\hline 105 & 1 & 10011000 & -1.212313 & 5.815214 & -0.185568 \\
\hline 106 & 1 & 10011000 & -0.701836 & 5.840449 & 1.523352 \\
\hline
\end{tabular}

$\left[\left(\mathrm{S}_{\mathrm{a}}\right) \text {-Binap } \mathrm{Au}\right]_{2}-\mathbf{I}$

\begin{tabular}{|c|c|c|c|c|c|}
\hline \multirow{2}{*}{$\begin{array}{l}\text { Center } \\
\text { Number }\end{array}$} & \multirow{2}{*}{$\begin{array}{l}\text { Atomic } \\
\text { Number }\end{array}$} & \multirow{2}{*}{$\begin{array}{l}\text { Atomic } \\
\text { Type }\end{array}$} & \multicolumn{3}{|c|}{ Coordinates (Angstroms) } \\
\hline & & & $\mathrm{x}$ & $\mathrm{Y}$ & $\mathrm{Z}$ \\
\hline & & & & - & . \\
\hline 1 & 6 & 10061000 & -1.773665 & 1.952728 & 0.078611 \\
\hline 2 & 6 & 10061002 & -0.323228 & 1.800377 & 0.371861 \\
\hline 3 & 6 & 10061002 & 0.103889 & 1.197734 & 1.491043 \\
\hline 4 & 6 & 10061000 & -0.858101 & 0.671161 & 2.474498 \\
\hline 5 & 6 & 10061002 & -0.398773 & 0.078050 & 3.745277 \\
\hline 6 & 6 & 10061000 & -1.290431 & -0.372418 & 4.643131 \\
\hline 7 & 6 & 10061000 & -2.667269 & -0.342679 & 4.383318 \\
\hline 8 & 6 & 10061002 & -3.153549 & 0.162916 & 3.236756 \\
\hline 9 & 6 & 10061000 & -2.229480 & 0.735485 & 2.220865 \\
\hline 10 & 6 & 10061000 & -2.709932 & 1.371692 & 0.969341 \\
\hline 11 & 6 & 10061002 & -4.164741 & 1.394033 & 0.683550 \\
\hline 12 & 6 & 10061000 & -4.726833 & 0.530808 & -0.205984 \\
\hline 13 & 6 & 10061000 & -6.089460 & 0.647403 & -0.556350 \\
\hline 14 & 6 & 10061002 & -6.891444 & 1.579781 & -0.019397 \\
\hline 15 & 6 & 10061000 & -6.372264 & 2.484921 & 1.017928 \\
\hline 16 & 6 & 10061000 & -5.033472 & 2.379565 & 1.394279 \\
\hline 17 & 6 & 10061002 & -4.555218 & 3.240370 & 2.508454 \\
\hline 18 & 6 & 10061000 & -5.396307 & 4.108743 & 3.099093 \\
\hline 19 & 6 & 10061000 & -6.734723 & 4.227203 & 2.692856 \\
\hline 20 & 6 & 10061002 & -7.237568 & 3.468854 & 1.702863 \\
\hline 21 & 6 & 10061000 & -4.192249 & 5.754663 & 0.323536 \\
\hline 22 & 6 & 10061000 & -5.309567 & 5.962794 & -0.487459 \\
\hline 23 & 6 & 10061000 & -5.518152 & 5.154573 & -1.606748 \\
\hline 24 & 6 & 10061000 & -4.606462 & 4.142234 & -1.918986 \\
\hline 25 & 6 & 10061000 & -3.483326 & 3.921461 & -1.109051 \\
\hline 26 & 6 & 10061000 & -3.286208 & 4.733068 & 0.022003 \\
\hline 27 & 15 & 10151003 & -2.319325 & 2.571645 & -1.588448 \\
\hline 28 & 6 & 10061000 & -0.935932 & 3.661190 & -2.137433 \\
\hline 29 & 6 & 10061000 & -0.294487 & 4.579350 & -1.286671 \\
\hline 30 & 6 & 10061000 & 0.635480 & 5.485586 & -1.802124 \\
\hline 31 & 6 & 10061000 & 0.965437 & 5.458918 & -3.158388 \\
\hline 32 & 6 & 10061000 & 0.353768 & 4.533473 & -4.005311 \\
\hline 33 & 6 & 10061000 & -0.603394 & 3.649785 & -3.499785 \\
\hline 34 & 15 & 10151003 & -3.780383 & -0.886669 & -0.901143 \\
\hline 35 & 6 & 10061000 & -4.708383 & -2.295539 & -0.190319 \\
\hline
\end{tabular}




\begin{tabular}{|c|c|c|c|c|c|}
\hline 36 & 6 & 10061000 & -4.239717 & -2.862699 & 1.000958 \\
\hline 37 & 6 & 10061000 & -4.903061 & -3.951055 & 1.573977 \\
\hline 38 & 6 & 10061000 & -6.047538 & -4.471208 & 0.964298 \\
\hline 39 & 6 & 10061000 & -6.533654 & -3.897111 & -0.213721 \\
\hline 40 & 6 & 10061000 & -5.871200 & -2.808173 & -0.788729 \\
\hline 41 & 6 & 10061000 & -4.103323 & -0.960564 & -2.702528 \\
\hline 42 & 6 & 10061000 & -4.446230 & 0.196484 & -3.417344 \\
\hline 43 & 6 & 10061000 & -4.730160 & 0.124864 & -4.783920 \\
\hline 44 & 6 & 10061000 & -4.685543 & -1.105262 & -5.444093 \\
\hline 45 & 6 & 10061000 & -4.345370 & -2.262369 & -4.739586 \\
\hline 46 & 6 & 10061000 & -4.034404 & -2.188314 & -3.378265 \\
\hline 47 & 1 & 10011000 & -3.358300 & -2.459831 & 1.481981 \\
\hline 48 & 1 & 10011000 & -4.531394 & -4.389985 & 2.490657 \\
\hline 49 & 1 & 10011000 & -6.560999 & -5.314500 & 1.407572 \\
\hline 50 & 1 & 10011000 & -7.424709 & -4.295609 & -0.681150 \\
\hline 51 & 1 & 10011000 & -6.266228 & -2.376055 & -1.697439 \\
\hline 52 & 1 & 10011000 & -3.762578 & -3.091789 & -2.849268 \\
\hline 53 & 1 & 10011000 & -4.311866 & -3.215737 & -5.250488 \\
\hline 54 & 1 & 10011000 & -4.914570 & -1.161947 & -6.500259 \\
\hline 55 & 1 & 10011000 & -4.995383 & 1.021846 & -5.328456 \\
\hline 56 & 1 & 10011000 & -4.507984 & 1.151258 & -2.920022 \\
\hline 57 & 1 & 10011000 & -0.499756 & 4.603000 & -0.229681 \\
\hline 58 & 1 & 10011000 & 1.096038 & 6.213872 & -1.148784 \\
\hline 59 & 1 & 10011000 & 1.686456 & 6.161370 & -3.554841 \\
\hline 60 & 1 & 10011000 & 0.601604 & 4.518009 & -5.058678 \\
\hline 61 & 1 & 10011000 & -1.108792 & 2.975664 & -4.178737 \\
\hline 62 & 1 & 10011000 & -6.514897 & -0.027945 & -1.285558 \\
\hline 63 & 1 & 10011000 & -7.932071 & 1.639711 & -0.323398 \\
\hline 64 & 1 & 10011000 & -8.278190 & 3.576265 & 1.412536 \\
\hline 65 & 1 & 10011000 & -7.375994 & 4.943233 & 3.190827 \\
\hline 66 & 1 & 10011000 & -5.031883 & 4.736395 & 3.902338 \\
\hline 67 & 1 & 10011000 & -3.525513 & 3.188837 & 2.845648 \\
\hline 68 & 1 & 10011000 & -4.225608 & 0.155304 & 3.070306 \\
\hline 69 & 1 & 10011000 & -3.351878 & -0.742582 & 5.120413 \\
\hline 70 & 1 & 10011000 & -0.938707 & -0.786018 & 5.578952 \\
\hline 71 & 1 & 10011000 & 0.660131 & 0.000185 & 3.949625 \\
\hline 72 & 1 & 10011000 & 1.167250 & 1.085115 & 1.669578 \\
\hline 73 & 1 & 10011000 & 0.417402 & 2.152594 & -0.332976 \\
\hline 74 & 1 & 10011000 & -6.016835 & 6.745050 & -0.244562 \\
\hline 75 & 1 & 10011000 & -4.040857 & 6.368883 & 1.201668 \\
\hline 76 & 1 & 10011000 & -2.469305 & 4.553449 & 0.702996 \\
\hline 77 & 1 & 10011000 & -4.775157 & 3.533494 & -2.797154 \\
\hline 78 & 1 & 10011000 & -6.384710 & 5.314368 & -2.235016 \\
\hline 79 & 79 & 10791004 & 3.047570 & 2.185344 & 0.390830 \\
\hline 80 & 79 & 10791004 & -1.428525 & -1.336184 & -0.190583 \\
\hline 81 & 6 & 10061000 & 1.939162 & -0.500217 & -1.747435 \\
\hline 82 & 6 & 10061002 & 1.099293 & 0.437168 & -2.536296 \\
\hline 83 & 6 & 10061002 & 0.143408 & -0.001618 & -3.365341 \\
\hline 84 & 6 & 10061000 & -0.097185 & -1.443062 & -3.529169 \\
\hline 85 & 6 & 10061002 & -0.987372 & -1.944822 & -4.597615 \\
\hline 86 & 6 & 10061000 & -1.136512 & -3.267354 & -4.784438 \\
\hline 87 & 6 & 10061000 & -0.474112 & -4.199544 & -3.975280 \\
\hline 88 & 6 & 10061002 & 0.354450 & -3.811366 & -2.991238 \\
\hline 89 & 6 & 10061000 & 0.587450 & -2.363688 & -2.730412 \\
\hline 90 & 6 & 10061000 & 1.604312 & -1.881169 & -1.754257 \\
\hline 91 & 6 & 10061000 & 2.334343 & -2.893821 & -0.971369 \\
\hline 92 & 6 & 10061000 & 1.790779 & -3.452179 & 0.204168 \\
\hline 93 & 6 & 10061002 & 2.575857 & -4.501371 & 0.907874 \\
\hline 94 & 6 & 10061000 & 3.705438 & -5.000637 & 0.386686 \\
\hline 95 & 6 & 10061000 & 4.192122 & -4.562877 & -0.847552 \\
\hline 96 & 6 & 10061000 & 3.536305 & -3.546123 & -1.559259 \\
\hline 97 & 6 & 10061002 & 4.073404 & -3.175312 & -2.894536 \\
\hline 98 & 6 & 10061000 & 5.184168 & -3.766218 & -3.366960 \\
\hline 99 & 6 & 10061000 & 5.857967 & -4.753497 & -2.634260 \\
\hline 100 & 6 & 10061002 & 5.414246 & -5.162333 & -1.433370 \\
\hline 101 & 6 & 10061000 & 6.424936 & -2.283016 & 0.288976 \\
\hline 102 & 6 & 10061000 & 6.020223 & -2.588256 & 1.590644 \\
\hline 103 & 6 & 10061000 & 4.835171 & -2.049724 & 2.096929 \\
\hline 104 & 6 & 10061000 & 4.048873 & -1.213210 & 1.298493 \\
\hline 105 & 6 & 10061000 & 4.431062 & -0.926947 & -0.017273 \\
\hline 106 & 6 & 10061000 & 5.639570 & -1.448302 & -0.511811 \\
\hline 107 & 15 & 10151003 & 3.434091 & 0.272793 & -0.969430 \\
\hline 108 & 6 & 10061000 & 4.512405 & 0.809233 & -2.350602 \\
\hline 109 & 6 & 10061000 & 5.638636 & 1.591187 & -2.055240 \\
\hline 110 & 6 & 10061000 & 6.485554 & 2.025865 & -3.078593 \\
\hline 111 & 6 & 10061000 & 6.213106 & 1.682307 & -4.404899 \\
\hline 112 & 6 & 10061000 & 5.092096 & 0.905781 & -4.708480 \\
\hline 113 & 6 & 10061000 & 4.241400 & 0.471616 & -3.687439 \\
\hline 114 & 15 & 10151003 & 0.066143 & -3.107361 & 0.771572 \\
\hline 115 & 6 & 10061000 & -0.805672 & -4.639655 & 0.272363 \\
\hline
\end{tabular}




\begin{tabular}{|c|c|c|c|c|c|}
\hline 116 & 6 & 10061000 & -1.827668 & -4.551023 & -0.682062 \\
\hline 117 & 6 & 10061000 & -2.538945 & -5.690329 & -1.065868 \\
\hline 118 & 6 & 10061000 & -2.234944 & -6.928140 & -0.494450 \\
\hline 119 & 6 & 10061000 & -1.220870 & -7.025105 & 0.462538 \\
\hline 120 & 6 & 10061000 & -0.508279 & -5.885190 & 0.847968 \\
\hline 121 & 6 & 10061000 & 0.040539 & -3.206320 & 2.604424 \\
\hline 122 & 6 & 10061000 & -1.103208 & -3.673972 & 3.270852 \\
\hline 123 & 6 & 10061000 & -1.105496 & -3.807346 & 4.662683 \\
\hline 124 & 6 & 10061000 & 0.031418 & -3.467159 & 5.400320 \\
\hline 125 & 6 & 10061000 & 1.164891 & -2.978031 & 4.747020 \\
\hline 126 & 6 & 10061000 & 1.165654 & -2.839590 & 3.356371 \\
\hline 127 & 1 & 10011000 & -2.069560 & -3.600116 & -1.134896 \\
\hline 128 & 1 & 10011000 & -3.326215 & -5.612376 & -1.804544 \\
\hline 129 & 1 & 10011000 & -2.787724 & -7.810615 & -0.789240 \\
\hline 130 & 1 & 10011000 & -0.993101 & -7.982522 & 0.912885 \\
\hline 131 & 1 & 10011000 & 0.247960 & -5.976195 & 1.612596 \\
\hline 132 & 1 & 10011000 & 2.046646 & -2.459472 & 2.869205 \\
\hline 133 & 1 & 10011000 & 2.043404 & -2.706809 & 5.316902 \\
\hline 134 & 1 & 10011000 & 0.030039 & -3.570888 & 6.477546 \\
\hline 135 & 1 & 10011000 & -1.987637 & -4.177528 & 5.168645 \\
\hline 136 & 1 & 10011000 & -1.984020 & -3.960671 & 2.715299 \\
\hline 137 & 1 & 10011000 & 5.859905 & 1.863283 & -1.030712 \\
\hline 138 & 1 & 10011000 & 7.352934 & 2.628738 & -2.843175 \\
\hline 139 & 1 & 10011000 & 6.869765 & 2.018033 & -5.197031 \\
\hline 140 & 1 & 10011000 & 4.881405 & 0.640370 & -5.736385 \\
\hline 141 & 1 & 10011000 & 3.383037 & -0.126133 & -3.952320 \\
\hline 142 & 1 & 10011000 & 2.236970 & -4.886480 & 1.861702 \\
\hline 143 & 1 & 10011000 & 4.237823 & -5.771108 & 0.931287 \\
\hline 144 & 1 & 10011000 & 5.956808 & -5.934159 & -0.895252 \\
\hline 145 & 1 & 10011000 & 6.751354 & -5.201573 & -3.050237 \\
\hline 146 & 1 & 10011000 & 5.566322 & -3.476862 & -4.337673 \\
\hline 147 & 1 & 10011000 & 3.574290 & -2.430738 & -3.501751 \\
\hline 148 & 1 & 10011000 & 0.852963 & -4.570773 & -2.398037 \\
\hline 149 & 1 & 10011000 & -0.629276 & -5.256297 & -4.152102 \\
\hline 150 & 1 & 10011000 & -1.783928 & -3.622847 & -5.575447 \\
\hline 151 & 1 & 10011000 & -1.512098 & -1.245655 & -5.241689 \\
\hline 152 & 1 & 10011000 & -0.425381 & 0.706997 & -3.956044 \\
\hline 153 & 1 & 10011000 & 1.296570 & 1.503293 & -2.483608 \\
\hline 154 & 1 & 10011000 & 6.626689 & -3.238011 & 2.207932 \\
\hline 155 & 1 & 10011000 & 7.344829 & -2.697614 & -0.102237 \\
\hline 156 & 1 & 10011000 & 5.971979 & -1.231140 & -1.516827 \\
\hline 157 & 1 & 10011000 & 3.147253 & -0.774870 & 1.705672 \\
\hline 158 & 1 & 10011000 & 4.529877 & -2.277132 & 3.109626 \\
\hline 159 & 6 & 10061000 & 2.434726 & 3.880173 & 2.877671 \\
\hline 160 & 1 & 10011000 & 2.111238 & 4.832345 & 3.305158 \\
\hline 161 & 6 & 10061000 & 3.092984 & 5.094703 & 0.948268 \\
\hline 162 & 6 & 10061000 & 3.587556 & 5.126875 & -0.383940 \\
\hline 163 & 8 & 10081000 & 3.788313 & 4.131001 & -1.166232 \\
\hline 164 & 6 & 10061000 & 2.471466 & 2.747020 & 3.792606 \\
\hline 165 & 6 & 10061000 & 3.361421 & 1.640255 & 3.654866 \\
\hline 166 & 6 & 10061000 & 1.667919 & 2.787681 & 4.963386 \\
\hline 167 & 6 & 10061000 & 3.413733 & 0.625006 & 4.612690 \\
\hline 168 & 1 & 10011000 & 4.055556 & 1.612073 & 2.820133 \\
\hline 169 & 6 & 10061000 & 1.713533 & 1.775204 & 5.935106 \\
\hline 170 & 1 & 10011000 & 0.996400 & 3.630996 & 5.113221 \\
\hline 171 & 6 & 10061000 & 2.586192 & 0.679495 & 5.756345 \\
\hline 172 & 1 & 10011000 & 4.113983 & -0.200078 & 4.524255 \\
\hline 173 & 1 & 10011000 & 1.079410 & 1.849844 & 6.812893 \\
\hline 174 & 7 & 10071000 & 2.856949 & 3.908853 & 1.608115 \\
\hline 175 & 1 & 10011000 & 3.002919 & 6.029340 & 1.491743 \\
\hline 176 & 8 & 10081003 & 2.710708 & -0.392549 & 6.639045 \\
\hline 177 & 8 & 10081003 & 3.863820 & 6.428604 & -0.802218 \\
\hline 178 & 6 & 10061003 & 1.904082 & -0.401468 & 7.853005 \\
\hline 179 & 1 & 10011000 & 2.177168 & -1.321434 & 8.372962 \\
\hline 180 & 1 & 10011000 & 2.133063 & 0.463948 & 8.488833 \\
\hline 181 & 1 & 10011000 & 0.830825 & -0.413629 & 7.615705 \\
\hline 182 & 6 & 10061003 & 4.501616 & 6.593889 & -2.113395 \\
\hline 183 & 1 & 10011000 & 5.581743 & 6.428767 & -2.023499 \\
\hline 184 & 1 & 10011000 & 4.088952 & 5.887240 & -2.838872 \\
\hline 185 & 1 & 10011000 & 4.298538 & 7.626285 & -2.404856 \\
\hline
\end{tabular}

$\left[\left(\mathrm{S}_{\mathrm{a}}\right) \text {-Binap } \mathrm{Au}\right]_{2}-\mathbf{I}-\mathbf{b}$

$\begin{array}{llll}\text { Center } & \text { Atomic } & \text { Atomic } & \text { Coordinates (Angstroms) } \\ \text { Number } & \text { Number } & \text { Type } & \mathrm{X}\end{array}$




\begin{tabular}{|c|c|c|c|c|c|}
\hline 1 & 6 & 10061002 & 1.564350 & -2.083639 & 0.427410 \\
\hline 2 & 6 & 10061002 & 0.424275 & -1.404476 & 1.112495 \\
\hline 3 & 6 & 10061002 & 0.596116 & -0.740937 & 2.265251 \\
\hline 4 & 6 & 10061000 & 1.928749 & -0.657853 & 2.878570 \\
\hline 5 & 6 & 10061002 & 2.129218 & -0.034029 & 4.203152 \\
\hline 6 & 6 & 10061000 & 3.370699 & 0.107496 & 4.697936 \\
\hline 7 & 6 & 10061000 & 4.496726 & -0.324584 & 3.982319 \\
\hline 8 & 6 & 10061002 & 4.380253 & -0.928824 & 2.786598 \\
\hline 9 & 6 & 10061000 & 3.035903 & -1.156214 & 2.190891 \\
\hline 10 & 6 & 10061002 & 2.831930 & -1.890406 & 0.907681 \\
\hline 11 & 6 & 10061002 & 4.027642 & -2.391919 & 0.180367 \\
\hline 12 & 6 & 10061000 & 4.505947 & -1.753933 & -0.922896 \\
\hline 13 & 6 & 10061000 & 5.568495 & -2.309414 & -1.668273 \\
\hline 14 & 6 & 10061002 & 6.178100 & -3.449719 & -1.308876 \\
\hline 15 & 6 & 10061000 & 5.782499 & -4.133842 & -0.067778 \\
\hline 16 & 6 & 10061000 & 4.737354 & -3.604346 & 0.688623 \\
\hline 17 & 6 & 10061002 & 4.402528 & -4.271720 & 1.974702 \\
\hline 18 & 6 & 10061000 & 5.082562 & -5.365328 & 2.364271 \\
\hline 19 & 6 & 10061000 & 6.117322 & -5.904048 & 1.583508 \\
\hline 20 & 6 & 10061002 & 6.480456 & -5.344103 & 0.416153 \\
\hline 21 & 6 & 10061000 & 2.711244 & -6.503415 & 0.129985 \\
\hline 22 & 6 & 10061000 & 3.453177 & -6.952369 & -0.964269 \\
\hline 23 & 6 & 10061000 & 3.540808 & -6.167414 & -2.115632 \\
\hline 24 & 6 & 10061000 & 2.881812 & -4.936252 & -2.175493 \\
\hline 25 & 6 & 10061000 & 2.140424 & -4.471641 & -1.079515 \\
\hline 26 & 6 & 10061000 & 2.062782 & -5.265551 & 0.078248 \\
\hline 27 & 15 & 10151003 & 1.256395 & -2.857915 & -1.244587 \\
\hline 28 & 6 & 10061000 & -0.437782 & -3.580374 & -1.098330 \\
\hline 29 & 6 & 10061000 & -0.956622 & -4.099480 & 0.103001 \\
\hline 30 & 6 & 10061000 & -2.155814 & -4.817360 & 0.100365 \\
\hline 31 & 6 & 10061000 & -2.861424 & -5.002190 & -1.089598 \\
\hline 32 & 6 & 10061000 & -2.374665 & -4.458014 & -2.279369 \\
\hline 33 & 6 & 10061000 & -1.164933 & -3.758427 & -2.284701 \\
\hline 34 & 15 & 10151003 & 3.896777 & -0.082705 & -1.397671 \\
\hline 35 & 6 & 10061000 & 5.331464 & 0.947851 & -0.919291 \\
\hline 36 & 6 & 10061000 & 5.246952 & 1.700091 & 0.258541 \\
\hline 37 & 6 & 10061000 & 6.323606 & 2.489922 & 0.670826 \\
\hline 38 & 6 & 10061000 & 7.495389 & 2.525228 & -0.089336 \\
\hline 39 & 6 & 10061000 & 7.592696 & 1.765388 & -1.258428 \\
\hline 40 & 6 & 10061000 & 6.517128 & 0.973041 & -1.671309 \\
\hline 41 & 6 & 10061000 & 3.827774 & -0.002605 & -3.224484 \\
\hline 42 & 6 & 10061000 & 3.421570 & -1.122933 & -3.962196 \\
\hline 43 & 6 & 10061000 & 3.402280 & -1.079530 & -5.359105 \\
\hline 44 & 6 & 10061000 & 3.783750 & 0.086461 & -6.027898 \\
\hline 45 & 6 & 10061000 & 4.170783 & 1.213889 & -5.298924 \\
\hline 46 & 6 & 10061000 & 4.174979 & 1.177088 & -3.901352 \\
\hline 47 & 1 & 10011000 & 4.348586 & 1.665255 & 0.860037 \\
\hline 48 & 1 & 10011000 & 6.252169 & 3.070334 & 1.581338 \\
\hline 49 & 1 & 10011000 & 8.329219 & 3.136932 & 0.229750 \\
\hline 50 & 1 & 10011000 & 8.502002 & 1.790145 & -1.844840 \\
\hline 51 & 1 & 10011000 & 6.609248 & 0.397908 & -2.581346 \\
\hline 52 & 1 & 10011000 & 4.459983 & 2.064330 & -3.352597 \\
\hline 53 & 1 & 10011000 & 4.454041 & 2.121311 & -5.816226 \\
\hline 54 & 1 & 10011000 & 3.771361 & 0.119129 & -7.109520 \\
\hline 55 & 1 & 10011000 & 3.093990 & -1.950489 & -5.922686 \\
\hline 56 & 1 & 10011000 & 3.129463 & -2.030953 & -3.458273 \\
\hline 57 & 1 & 10011000 & -0.438693 & -3.973238 & 1.040754 \\
\hline 58 & 1 & 10011000 & -2.532909 & -5.242234 & 1.020549 \\
\hline 59 & 1 & 10011000 & -3.791559 & -5.555593 & -1.087254 \\
\hline 60 & 1 & 10011000 & -2.925165 & -4.594430 & -3.201103 \\
\hline 61 & 1 & 10011000 & -0.776761 & -3.383147 & -3.223008 \\
\hline 62 & 1 & 10011000 & 5.914391 & -1.812640 & -2.563440 \\
\hline 63 & 1 & 10011000 & 6.987879 & -3.845370 & -1.914277 \\
\hline 64 & 1 & 10011000 & 7.286558 & -5.779211 & -0.166522 \\
\hline 65 & 1 & 10011000 & 6.634327 & -6.789421 & 1.930932 \\
\hline 66 & 1 & 10011000 & 4.821660 & -5.846660 & 3.298177 \\
\hline 67 & 1 & 10011000 & 3.600640 & -3.896635 & 2.601473 \\
\hline 68 & 1 & 10011000 & 5.278252 & -1.245210 & 2.266453 \\
\hline 69 & 1 & 10011000 & 5.481094 & -0.172964 & 4.406638 \\
\hline 70 & 1 & 10011000 & 3.506604 & 0.571212 & 5.666583 \\
\hline 71 & 1 & 10011000 & 1.274286 & 0.304621 & 4.779490 \\
\hline 72 & 1 & 10011000 & -0.241649 & -0.237895 & 2.733526 \\
\hline 73 & 1 & 10011000 & -0.562901 & -1.417456 & 0.665764 \\
\hline 74 & 1 & 10011000 & 3.965131 & -7.904629 & -0.917368 \\
\hline 75 & 1 & 10011000 & 2.657054 & -7.104294 & 1.028492 \\
\hline 76 & 1 & 10011000 & 1.546050 & -4.919321 & 0.957997 \\
\hline 77 & 1 & 10011000 & 2.944605 & -4.349693 & -3.081503 \\
\hline 78 & 1 & 10011000 & 4.115747 & -6.514700 & -2.964152 \\
\hline 79 & 79 & 10791004 & -3.345745 & -1.256329 & 1.317588 \\
\hline 80 & 79 & 10791004 & 1.972035 & 0.983592 & -0.238912 \\
\hline
\end{tabular}




\begin{tabular}{|c|c|c|c|c|c|}
\hline 81 & 6 & 10061000 & -1.844094 & 1.098259 & -0.892981 \\
\hline 82 & 6 & 10061002 & -1.503826 & -0.077765 & -1.732578 \\
\hline 83 & 6 & 10061002 & -0.646619 & 0.012541 & -2.757680 \\
\hline 84 & 6 & 10061000 & -0.030497 & 1.304393 & -3.100767 \\
\hline 85 & 6 & 10061002 & 0.741758 & 1.466399 & -4.351306 \\
\hline 86 & 6 & 10061000 & 1.213882 & 2.675416 & -4.699396 \\
\hline 87 & 6 & 10061000 & 1.008062 & 3.801612 & -3.891796 \\
\hline 88 & 6 & 10061002 & 0.316569 & 3.724366 & -2.742006 \\
\hline 89 & 6 & 10061000 & -0.248552 & 2.423629 & -2.290246 \\
\hline 90 & 6 & 10061000 & -1.127298 & 2.308780 & -1.092996 \\
\hline 91 & 6 & 10061002 & -1.327121 & 3.512078 & -0.256888 \\
\hline 92 & 6 & 10061002 & -0.431085 & 3.879707 & 0.701052 \\
\hline 93 & 6 & 10061002 & -0.732874 & 5.080917 & 1.535452 \\
\hline 94 & 6 & 10061000 & -1.786817 & 5.867561 & 1.275809 \\
\hline 95 & 6 & 10061000 & -2.636236 & 5.595829 & 0.202331 \\
\hline 96 & 6 & 10061000 & -2.432099 & 4.458861 & -0.593949 \\
\hline 97 & 6 & 10061002 & -3.314017 & 4.273154 & -1.774495 \\
\hline 98 & 6 & 10061000 & -4.323227 & 5.129190 & -2.009513 \\
\hline 99 & 6 & 10061000 & -4.559962 & 6.230579 & -1.174509 \\
\hline 100 & 6 & 10061002 & -3.773624 & 6.490695 & -0.115792 \\
\hline 101 & 6 & 10061000 & -4.749462 & 4.101408 & 2.310491 \\
\hline 102 & 6 & 10061000 & -3.978486 & 4.065148 & 3.473956 \\
\hline 103 & 6 & 10061000 & -2.995535 & 3.085549 & 3.627604 \\
\hline 104 & 6 & 10061000 & -2.771443 & 2.151282 & 2.610935 \\
\hline 105 & 6 & 10061000 & -3.521389 & 2.195145 & 1.430196 \\
\hline 106 & 6 & 10061000 & -4.531085 & 3.164978 & 1.295603 \\
\hline 107 & 15 & 10151003 & -3.309051 & 0.844931 & 0.212243 \\
\hline 108 & 6 & 10061000 & -4.793247 & 0.907837 & -0.863755 \\
\hline 109 & 6 & 10061000 & -6.053116 & 0.698795 & -0.281556 \\
\hline 110 & 6 & 10061000 & -7.210434 & 0.742180 & -1.063762 \\
\hline 111 & 6 & 10061000 & -7.117125 & 0.979257 & -2.436957 \\
\hline 112 & 6 & 10061000 & -5.865030 & 1.158201 & -3.030405 \\
\hline 113 & 6 & 10061000 & -4.705034 & 1.113302 & -2.250536 \\
\hline 114 & 15 & 10151003 & 1.240095 & 3.094699 & 0.872387 \\
\hline 115 & 6 & 10061000 & 2.320022 & 4.339172 & 0.067958 \\
\hline 116 & 6 & 10061000 & 3.069879 & 3.959364 & -1.053724 \\
\hline 117 & 6 & 10061000 & 3.918686 & 4.874903 & -1.680797 \\
\hline 118 & 6 & 10061000 & 4.027527 & 6.177231 & -1.187899 \\
\hline 119 & 6 & 10061000 & 3.289924 & 6.562472 & -0.065289 \\
\hline 120 & 6 & 10061000 & 2.440377 & 5.647300 & 0.563907 \\
\hline 121 & 6 & 10061000 & 1.742209 & 3.201223 & 2.633651 \\
\hline 122 & 6 & 10061000 & 3.080030 & 3.447622 & 2.982492 \\
\hline 123 & 6 & 10061000 & 3.449471 & 3.566217 & 4.325587 \\
\hline 124 & 6 & 10061000 & 2.487564 & 3.433926 & 5.330209 \\
\hline 125 & 6 & 10061000 & 1.158738 & 3.167880 & 4.991638 \\
\hline 126 & 6 & 10061000 & 0.792214 & 3.038407 & 3.649020 \\
\hline 127 & 1 & 10011000 & 2.989705 & 2.957449 & -1.451103 \\
\hline 128 & 1 & 10011000 & 4.490922 & 4.574799 & -2.548960 \\
\hline 129 & 1 & 10011000 & 4.685969 & 6.886216 & -1.672713 \\
\hline 130 & 1 & 10011000 & 3.382323 & 7.568686 & 0.322484 \\
\hline 131 & 1 & 10011000 & 1.904599 & 5.956846 & 1.447715 \\
\hline 132 & 1 & 10011000 & -0.230722 & 2.821197 & 3.399926 \\
\hline 133 & 1 & 10011000 & 0.414520 & 3.051310 & 5.768682 \\
\hline 134 & 1 & 10011000 & 2.773575 & 3.526447 & 6.369792 \\
\hline 135 & 1 & 10011000 & 4.481034 & 3.762966 & 4.587065 \\
\hline 136 & 1 & 10011000 & 3.835413 & 3.568536 & 2.220043 \\
\hline 137 & 1 & 10011000 & -6.137980 & 0.508921 & 0.781091 \\
\hline 138 & 1 & 10011000 & -8.178600 & 0.585099 & -0.606396 \\
\hline 139 & 1 & 10011000 & -8.013149 & 1.007343 & -3.043309 \\
\hline 140 & 1 & 10011000 & -5.792430 & 1. 323392 & -4.097460 \\
\hline 141 & 1 & 10011000 & -3.750537 & 1.233116 & -2.739300 \\
\hline 142 & 1 & 10011000 & -0.094292 & 5.336529 & 2.372406 \\
\hline 143 & 1 & 10011000 & -1.966125 & 6.733815 & 1.901012 \\
\hline 144 & 1 & 10011000 & -3.977553 & 7.358391 & 0.504727 \\
\hline 145 & 1 & 10011000 & -5.386570 & 6.893266 & -1.397159 \\
\hline 146 & 1 & 10011000 & -4.962521 & 4.971514 & -2.868963 \\
\hline 147 & 1 & 10011000 & -3.140508 & 3.460272 & -2.467203 \\
\hline 148 & 1 & 10011000 & 0.172721 & 4.625860 & -2.155604 \\
\hline 149 & 1 & 10011000 & 1.410912 & 4.756285 & -4.205422 \\
\hline 150 & 1 & 10011000 & 1.763806 & 2.784973 & -5.625022 \\
\hline 151 & 1 & 10011000 & 0.909930 & 0.613540 & -5.001260 \\
\hline 152 & 1 & 10011000 & -0.440878 & -0.862534 & -3.365824 \\
\hline 153 & 1 & 10011000 & -1.985836 & -1.029765 & -1.533930 \\
\hline 154 & 1 & 10011000 & -4.148588 & 4.789818 & 4.259413 \\
\hline 155 & 1 & 10011000 & -5.515123 & 4.857137 & 2.192642 \\
\hline 156 & 1 & 10011000 & -5.139128 & 3.218671 & 0.404388 \\
\hline 157 & 1 & 10011000 & -2.031098 & 1.376264 & 2.753038 \\
\hline 158 & 1 & 10011000 & -2.414646 & 3.043757 & 4.539748 \\
\hline 159 & 6 & 10061000 & -5.399567 & -3.569940 & 1.385362 \\
\hline 160 & 1 & 10011000 & -5.955066 & -4.338177 & 1.930776 \\
\hline
\end{tabular}




$\begin{array}{lrrrrr}161 & 6 & 10061000 & -3.734366 & -3.680024 & 3.081570 \\ 162 & 6 & 10061000 & -2.564385 & -3.116967 & 3.628993 \\ 163 & 8 & 10081000 & -1.966378 & -2.053971 & 3.203471 \\ 164 & 6 & 10061000 & -5.964709 & -3.166467 & 0.099972 \\ 165 & 6 & 10061000 & -5.203038 & -2.642260 & -0.984248 \\ 166 & 6 & 10061000 & -7.349870 & -3.374065 & -0.127071 \\ 167 & 6 & 10061000 & -5.795670 & -2.326208 & -2.208071 \\ 168 & 1 & 10011000 & -4.130511 & -2.520520 & -0.873730 \\ 169 & 6 & 10061000 & -7.963577 & -3.041257 & -1.345104 \\ 170 & 1 & 10011000 & -7.959664 & -3.790414 & 0.672397 \\ 171 & 6 & 10061000 & -7.183905 & -2.508785 & -2.394204 \\ 172 & 1 & 10011000 & -5.207998 & -1.955623 & -3.043224 \\ 173 & 1 & 10011000 & -9.029774 & -3.203450 & -1.466170 \\ 174 & 7 & 10071000 & -4.280816 & -3.118581 & 1.946756 \\ 175 & 1 & 10011000 & -4.166109 & -4.575332 & 3.515673 \\ 176 & 8 & 10081003 & -7.674413 & -2.140915 & -3.644486 \\ 177 & 8 & 10081003 & -2.044209 & -3.816656 & 4.713941 \\ 178 & 6 & 10061003 & -9.102185 & -2.281361 & -3.908934 \\ 179 & 1 & 10011000 & -9.241594 & -1.924257 & -4.930708 \\ 180 & 1 & 10011000 & -9.417609 & -3.330866 & -3.837573 \\ 181 & 1 & 10011000 & -9.693945 & -1.666728 & -3.216765 \\ 182 & 6 & 10061003 & -0.812495 & -3.286313 & 5.305998 \\ 183 & 1 & 10011000 & -0.594211 & -3.953246 & 6.142211 \\ 184 & 1 & 10011000 & 0.004610 & -3.299872 & 4.575073 \\ 185 & 1 & 10011000 & -0.960292 & -2.258676 & 5.655554 \\ -----------------------------------------------------------\end{array}$

\section{[( $\left.\mathrm{S}_{\mathrm{a}}\right)$-Binap- $\left(\mathrm{R}_{\mathrm{a}}\right)$-Binap-Au $\left.\mathrm{Bu}_{2}\right]$-I}

\begin{tabular}{|c|c|c|c|c|c|}
\hline \multirow{2}{*}{$\begin{array}{l}\text { Center } \\
\text { Number }\end{array}$} & \multirow{2}{*}{$\begin{array}{l}\text { Atomic } \\
\text { Number }\end{array}$} & \multirow{2}{*}{$\begin{array}{c}\text { Atomic } \\
\text { Type }\end{array}$} & \multicolumn{3}{|c|}{ Coordinates (Angstroms) } \\
\hline & & & $\mathrm{X}$ & $\mathrm{Y}$ & $\mathrm{Z}$ \\
\hline & & & & & -ー---ー-ー \\
\hline 1 & 6 & 10061000 & -2.232152 & -0.995464 & 0.579621 \\
\hline 2 & 6 & 10061000 & -0.925945 & -1.271079 & 1.214214 \\
\hline 3 & 6 & 10061000 & -0.623922 & -0.821435 & 2.494462 \\
\hline 4 & 6 & 10061000 & -1.548933 & -0.093897 & 3.239122 \\
\hline 5 & 6 & 10061000 & -1.246605 & 0.347786 & 4.532437 \\
\hline 6 & 6 & 10061000 & -2.200262 & 1.021237 & 5.302912 \\
\hline 7 & 6 & 10061000 & -3.492437 & 1.262402 & 4.825379 \\
\hline 8 & 6 & 10061000 & -3.870941 & 0.866438 & 3.543889 \\
\hline 9 & 6 & 10061002 & -2.882916 & 0.200838 & 2.666282 \\
\hline 10 & 6 & 10061000 & -3.187061 & -0.210098 & 1.269174 \\
\hline 11 & 6 & 10061000 & -4.443678 & 0.252402 & 0.637784 \\
\hline 12 & 6 & 10061000 & -4.501787 & 1.467534 & -0.075779 \\
\hline 13 & 6 & 10061000 & -5.736122 & 1.800585 & -0.828451 \\
\hline 14 & 6 & 10061000 & -6.875712 & 1.001795 & -0.756386 \\
\hline 15 & 6 & 10061000 & -6.908638 & -0.128259 & 0.059595 \\
\hline 16 & 6 & 10061002 & -5.702928 & -0.511723 & 0.829645 \\
\hline 17 & 6 & 10061000 & -5.847296 & -1.591136 & 1.829010 \\
\hline 18 & 6 & 10061000 & -7.045337 & -2.299162 & 1.903609 \\
\hline 19 & 6 & 10061000 & -8.128556 & -1.970946 & 1.081809 \\
\hline 20 & 6 & 10061000 & -8.071463 & -0.897174 & 0.186182 \\
\hline 21 & 6 & 10061000 & -4.687066 & 5.973557 & -1.954869 \\
\hline 22 & 6 & 10061000 & -3.757482 & 6.178502 & -2.978042 \\
\hline 23 & 6 & 10061000 & -2.656816 & 5.327204 & -3.101350 \\
\hline 24 & 6 & 10061000 & -2.488811 & 4.268068 & -2.205506 \\
\hline 25 & 6 & 10061000 & -3.424846 & 4.045007 & -1.186862 \\
\hline 26 & 6 & 10061000 & -4.518950 & 4.915696 & -1.055822 \\
\hline 27 & 15 & 10151003 & -3.118520 & 2.680351 & -0.009579 \\
\hline 28 & 6 & 10061000 & -3.221369 & 3.480938 & 1.624698 \\
\hline 29 & 6 & 10061000 & -4.456410 & 3.801475 & 2.208023 \\
\hline 30 & 6 & 10061000 & -4.499471 & 4.408316 & 3.467295 \\
\hline 31 & 6 & 10061000 & -3.312516 & 4.689938 & 4.150723 \\
\hline 32 & 6 & 10061000 & -2.080833 & 4.363139 & 3.576307 \\
\hline 33 & 6 & 10061000 & -2.035614 & 3.761035 & 2.316100 \\
\hline 34 & 15 & 10151003 & -2.513510 & -1.842528 & -1.029507 \\
\hline 35 & 6 & 10061000 & -3.775110 & -3.136888 & -0.751538 \\
\hline 36 & 6 & 10061000 & -4.408751 & -3.744623 & -1.846500 \\
\hline 37 & 6 & 10061000 & -5.304147 & -4.799877 & -1.648165 \\
\hline 38 & 6 & 10061000 & -5.533462 & -5.289761 & -0.360231 \\
\hline 39 & 6 & 10061000 & -4.850778 & -4.738468 & 0.726213 \\
\hline 40 & 6 & 10061000 & -3.961714 & -3.677732 & 0.529289 \\
\hline 41 & 6 & 10061000 & -3.138378 & -0.594046 & -2.211462 \\
\hline 42 & 6 & 10061000 & -2.223228 & 0.317384 & -2.755169 \\
\hline 43 & 6 & 10061000 & -2.632498 & 1.236142 & -3.725242 \\
\hline 44 & 6 & 10061000 & -3.962069 & 1.257160 & -4.151952 \\
\hline 45 & 6 & 10061000 & -4.880468 & 0.350676 & -3.616781 \\
\hline
\end{tabular}




\begin{tabular}{|c|c|c|c|c|c|}
\hline 46 & 6 & 10061000 & -4.472674 & -0.572984 & -2.648703 \\
\hline 47 & 1 & 10011000 & -4.207502 & -3.405633 & -2.854472 \\
\hline 48 & 1 & 10011000 & -5.807735 & -5.248169 & -2.494759 \\
\hline 49 & 1 & 10011000 & -6.221942 & -6.110630 & -0.207300 \\
\hline 50 & 1 & 10011000 & -5.003673 & -5.137630 & 1.720494 \\
\hline 51 & 1 & 10011000 & -3.412923 & -3.291311 & 1.377035 \\
\hline 52 & 1 & 10011000 & -5.208404 & -1.241362 & -2.227635 \\
\hline 53 & 1 & 10011000 & -5.912489 & 0.371839 & -3.941993 \\
\hline 54 & 1 & 10011000 & -4.280005 & 1.974487 & -4.897293 \\
\hline 55 & 1 & 10011000 & -1.917698 & 1.929339 & -4.149051 \\
\hline 56 & 1 & 10011000 & -1.187993 & 0.303229 & -2.439803 \\
\hline 57 & 1 & 10011000 & -5.383394 & 3.561083 & 1.705479 \\
\hline 58 & 1 & 10011000 & -5.453065 & 4.649519 & 3.918491 \\
\hline 59 & 1 & 10011000 & -3.347644 & 5.153177 & 5.128112 \\
\hline 60 & 1 & 10011000 & -1.161665 & 4.574122 & 4.107533 \\
\hline 61 & 1 & 10011000 & -1.077788 & 3.515566 & 1.878130 \\
\hline 62 & 1 & 10011000 & -5.761819 & 2.651946 & -1.488781 \\
\hline 63 & 1 & 10011000 & -7.748054 & 1.283621 & -1.333998 \\
\hline 64 & 1 & 10011000 & -8.953619 & -0.653149 & -0.393747 \\
\hline 65 & 1 & 10011000 & -9.044015 & -2.542748 & 1.162794 \\
\hline 66 & 1 & 10011000 & -7.139767 & -3.112450 & 2.611458 \\
\hline 67 & 1 & 10011000 & -5.035408 & -1.844989 & 2.496708 \\
\hline 68 & 1 & 10011000 & -4.877877 & 1.064077 & 3.201845 \\
\hline 69 & 1 & 10011000 & -4.208545 & 1.769995 & 5.458584 \\
\hline 70 & 1 & 10011000 & -1.936824 & 1.349153 & 6.300188 \\
\hline 71 & 1 & 10011000 & -0.268206 & 0.161278 & 4.958884 \\
\hline 72 & 1 & 10011000 & 0.338344 & -1.067850 & 2.915267 \\
\hline 73 & 1 & 10011000 & -0.184474 & -1.854034 & 0.682063 \\
\hline 74 & 1 & 10011000 & -3.887024 & 6.999633 & -3.671064 \\
\hline 75 & 1 & 10011000 & -5.536606 & 6.636821 & -1.856110 \\
\hline 76 & 1 & 10011000 & -5.247649 & 4.777003 & -0.271972 \\
\hline 77 & 1 & 10011000 & -1.622668 & 3.626382 & -2.301212 \\
\hline 78 & 1 & 10011000 & -1.929727 & 5.491640 & -3.886098 \\
\hline 79 & 79 & 10791004 & -0.800300 & 1.981390 & -0.308543 \\
\hline 80 & 79 & 10791004 & -0.529092 & -2.873009 & -1.798286 \\
\hline 81 & 6 & 10061000 & 2.673824 & 1. 345111 & -1.282832 \\
\hline 82 & 6 & 10061000 & 2.170294 & 0.839322 & -2.579634 \\
\hline 83 & 6 & 10061000 & 2.966604 & 0.090199 & -3.439409 \\
\hline 84 & 6 & 10061000 & 4.276144 & -0.238979 & -3.099214 \\
\hline 85 & 6 & 10061000 & 5.077619 & -0.991980 & -3.965092 \\
\hline 86 & 6 & 10061000 & 6.393346 & -1.321885 & -3.624632 \\
\hline 87 & 6 & 10061000 & 6.963989 & -0.910800 & -2.416346 \\
\hline 88 & 6 & 10061000 & 6.236766 & -0.156856 & -1.497720 \\
\hline 89 & 6 & 10061002 & 4.834056 & 0.212274 & -1.802574 \\
\hline 90 & 6 & 10061000 & 3.980176 & 0.997830 & -0.867130 \\
\hline 91 & 6 & 10061000 & 4.545158 & 1.379262 & 0.446934 \\
\hline 92 & 6 & 10061000 & 4.331345 & 0.579421 & 1.590491 \\
\hline 93 & 6 & 10061000 & 5.096052 & 0.877805 & 2.829805 \\
\hline 94 & 6 & 10061000 & 5.978043 & 1.953758 & 2.905552 \\
\hline 95 & 6 & 10061000 & 6.211082 & 2.774093 & 1.802707 \\
\hline 96 & 6 & 10061002 & 5.528617 & 2.490221 & 0.519914 \\
\hline 97 & 6 & 10061000 & 5.831372 & 3.370324 & -0.630805 \\
\hline 98 & 6 & 10061000 & 6.715330 & 4.434253 & -0.464306 \\
\hline 99 & 6 & 10061000 & 7.329158 & 4.675494 & 0.769250 \\
\hline 100 & 6 & 10061000 & 7.088734 & 3.861742 & 1.882088 \\
\hline 101 & 6 & 10061000 & 3.369927 & 4.617383 & 2.623400 \\
\hline 102 & 6 & 10061000 & 2.807903 & 4.027803 & 3.758245 \\
\hline 103 & 6 & 10061000 & 1.899122 & 2.975999 & 3.620287 \\
\hline 104 & 6 & 10061000 & 1.573644 & 2.493049 & 2.349059 \\
\hline 105 & 6 & 10061000 & 2.145703 & 3.068635 & 1.207531 \\
\hline 106 & 6 & 10061000 & 3.025490 & 4.153761 & 1.350619 \\
\hline 107 & 15 & 10151003 & 1.560624 & 2.535980 & -0.436011 \\
\hline 108 & 6 & 10061000 & 1.597224 & 4.074608 & -1.424763 \\
\hline 109 & 6 & 10061000 & 0.697707 & 5.100057 & -1.100281 \\
\hline 110 & 6 & 10061000 & 0.682589 & 6.285649 & -1.839856 \\
\hline 111 & 6 & 10061000 & 1.571064 & 6.456721 & -2.904391 \\
\hline 112 & 6 & 10061000 & 2.475910 & 5.442190 & -3.228982 \\
\hline 113 & 6 & 10061000 & 2.490670 & 4.252904 & -2.493687 \\
\hline 114 & 15 & 10151003 & 3.125735 & -0.835948 & 1.495493 \\
\hline 115 & 6 & 10061000 & 4.289016 & -2.007717 & 0.681632 \\
\hline 116 & 6 & 10061000 & 3.892007 & -2.637270 & -0.503469 \\
\hline 117 & 6 & 10061000 & 4.762919 & -3.508497 & -1.164885 \\
\hline 118 & 6 & 10061000 & 6.032697 & -3.761300 & -0.640351 \\
\hline 119 & 6 & 10061000 & 6.438155 & -3.133545 & 0.540209 \\
\hline 120 & 6 & 10061000 & 5.573543 & -2.253975 & 1.197892 \\
\hline 121 & 6 & 10061000 & 3.126561 & -1.512827 & 3.220404 \\
\hline 122 & 6 & 10061000 & 3.403963 & -2.867608 & 3.485215 \\
\hline 123 & 6 & 10061000 & 3.311957 & -3.368195 & 4.787313 \\
\hline 124 & 6 & 10061000 & 2.902321 & -2.536521 & 5.830986 \\
\hline 125 & 6 & 10061000 & 2.575417 & -1.204591 & 5.571744 \\
\hline
\end{tabular}




\begin{tabular}{|c|c|c|c|c|c|}
\hline 126 & 6 & 10061000 & 2.674114 & -0.701095 & 4.272264 \\
\hline 127 & 1 & 10011000 & 2.918060 & -2.429544 & -0.925974 \\
\hline 128 & 1 & 10011000 & 4.460142 & -3.978686 & -2.090264 \\
\hline 129 & 1 & 10011000 & 6.706206 & -4.434195 & -1.154725 \\
\hline 130 & 1 & 10011000 & 7.424692 & -3.323531 & 0.942345 \\
\hline 131 & 1 & 10011000 & 5.902359 & -1.774187 & 2.109643 \\
\hline 132 & 1 & 10011000 & 2.397846 & 0.323210 & 4.083354 \\
\hline 133 & 1 & 10011000 & 2.236567 & -0.563752 & 6.375409 \\
\hline 134 & 1 & 10011000 & 2.822599 & -2.928401 & 6.836655 \\
\hline 135 & 1 & 10011000 & 3.541719 & -4.407340 & 4.984139 \\
\hline 136 & 1 & 10011000 & 3.670601 & -3.551258 & 2.693041 \\
\hline 137 & 1 & 10011000 & 0.001986 & 4.976549 & -0.279322 \\
\hline 138 & 1 & 10011000 & -0.019931 & 7.069961 & -1.589380 \\
\hline 139 & 1 & 10011000 & 1.559095 & 7.375050 & -3.476922 \\
\hline 140 & 1 & 10011000 & 3.165981 & 5.576950 & -4.051816 \\
\hline 141 & 1 & 10011000 & 3.201468 & 3.485509 & -2.762375 \\
\hline 142 & 1 & 10011000 & 5.021550 & 0.240893 & 3.695616 \\
\hline 143 & 1 & 10011000 & 6.498283 & 2.135880 & 3.838513 \\
\hline 144 & 1 & 10011000 & 7.594585 & 4.088246 & 2.813154 \\
\hline 145 & 1 & 10011000 & 8.011050 & 5.510733 & 0.864151 \\
\hline 146 & 1 & 10011000 & 6.930026 & 5.084066 & -1.302849 \\
\hline 147 & 1 & 10011000 & 5.369931 & 3.207780 & -1.594903 \\
\hline 148 & 1 & 10011000 & 6.703715 & 0.132776 & -0.566229 \\
\hline 149 & 1 & 10011000 & 7.984510 & -1.188899 & -2.187073 \\
\hline 150 & 1 & 10011000 & 6.985233 & -1.909602 & -4.314373 \\
\hline 151 & 1 & 10011000 & 4.685725 & -1.328467 & -4.917148 \\
\hline 152 & 1 & 10011000 & 2.554350 & -0.223647 & -4.390295 \\
\hline 153 & 1 & 10011000 & 1.165190 & 1.090406 & -2.893771 \\
\hline 154 & 1 & 10011000 & 3.066386 & 4.394246 & 4.743096 \\
\hline 155 & 1 & 10011000 & 4.064604 & 5.440528 & 2.728796 \\
\hline 156 & 1 & 10011000 & 3.450166 & 4.638975 & 0.482411 \\
\hline 157 & 1 & 10011000 & 0.859432 & 1.686056 & 2.252774 \\
\hline 158 & 1 & 10011000 & 1.442807 & 2.538082 & 4.498609 \\
\hline 159 & 7 & 10071000 & 1.135359 & -4.190522 & -2.103383 \\
\hline 160 & 6 & 10061000 & 1.564375 & -5.039611 & -1.178108 \\
\hline 161 & 6 & 10061000 & 1.757183 & -4.046706 & -3.334308 \\
\hline 162 & 1 & 10011000 & 2.489134 & -5.582888 & -1.392675 \\
\hline 163 & 1 & 10011000 & 2.543832 & -4.747125 & -3.589776 \\
\hline 164 & 6 & 10061000 & 0.990051 & -5.254415 & 0.153653 \\
\hline 165 & 6 & 10061000 & 1.872578 & -5.533993 & 1.226816 \\
\hline 166 & 6 & 10061000 & -0.402490 & -5.223940 & 0.445859 \\
\hline 167 & 6 & 10061000 & 1.412712 & -5.700689 & 2.544392 \\
\hline 168 & 1 & 10011000 & 2.941001 & -5.602125 & 1.029077 \\
\hline 169 & 6 & 10061000 & -0.873999 & -5.388410 & 1.751310 \\
\hline 170 & 1 & 10011000 & -1.114666 & -5.118152 & -0.366754 \\
\hline 171 & 6 & 10061000 & 0.031160 & -5.604854 & 2.813323 \\
\hline 172 & 1 & 10011000 & 2.125156 & -5.907024 & 3.336697 \\
\hline 173 & 1 & 10011000 & -1.936523 & -5.393775 & 1.975809 \\
\hline 174 & 8 & 10081000 & -0.545599 & -5.732482 & 4.076198 \\
\hline 175 & 6 & 10061003 & 0.311573 & -6.040520 & 5.214665 \\
\hline 176 & 1 & 10011000 & -0.363925 & -6.131254 & 6.067101 \\
\hline 177 & 1 & 10011000 & 0.846524 & -6.987881 & 5.064968 \\
\hline 178 & 1 & 10011000 & 1.032271 & -5.232178 & 5.399224 \\
\hline 179 & 6 & 10061000 & 1.384071 & -2.980733 & -4.188486 \\
\hline 180 & 8 & 10081000 & 0.479458 & -2.102322 & -3.930174 \\
\hline 181 & 8 & 10081003 & 2.032305 & -2.815046 & -5.416795 \\
\hline 182 & 6 & 10061003 & 3.047571 & -3.770325 & -5.856353 \\
\hline 183 & 1 & 10011000 & 2.627289 & -4.779756 & -5.953848 \\
\hline 184 & 1 & 10011000 & 3.370620 & -3.409581 & -6.834813 \\
\hline 185 & 1 & 10011000 & 3.904627 & -3.789338 & -5.169138 \\
\hline
\end{tabular}

\section{$(S, S, S, R)-\mathbf{I I}$}

\begin{tabular}{|c|c|c|c|c|c|}
\hline \multirow{2}{*}{$\begin{array}{l}\text { Center } \\
\text { Number }\end{array}$} & \multirow{2}{*}{$\begin{array}{l}\text { Atomic } \\
\text { Number }\end{array}$} & \multirow{2}{*}{$\begin{array}{c}\text { Atomic } \\
\text { Type }\end{array}$} & \multicolumn{3}{|c|}{ Coordinates (Angstroms) } \\
\hline & & & $\mathrm{x}$ & $\mathrm{Y}$ & $\mathrm{Z}$ \\
\hline 1 & 6 & 10061000 & -1.031173 & 2.381041 & -0.221093 \\
\hline 2 & 6 & 10061002 & 0.178792 & 1.533083 & -0.060719 \\
\hline 3 & 6 & 10061002 & 0.461970 & 0.932534 & 1.105556 \\
\hline 4 & 6 & 10061000 & -0.424781 & 1.094250 & 2.269634 \\
\hline 5 & 6 & 10061002 & -0.097150 & 0.472647 & 3.567229 \\
\hline 6 & 6 & 10061000 & -0.906575 & 0.648531 & 4.624834 \\
\hline 7 & 6 & 10061000 & -2.098809 & 1.377646 & 4.518893 \\
\hline 8 & 6 & 10061002 & -2.470228 & 1.949795 & 3.360610 \\
\hline 9 & 6 & 10061000 & -1.598642 & 1.840639 & 2.159859 \\
\hline 10 & 6 & 10061000 & -1.933249 & 2.483477 & 0.865686 \\
\hline
\end{tabular}




\begin{tabular}{|c|c|c|c|c|c|}
\hline 11 & 6 & 10061002 & -3.219330 & 3.205715 & 0.731667 \\
\hline 12 & 6 & 10061000 & -4.288348 & 2.637560 & 0.110638 \\
\hline 13 & 6 & 10061000 & -5.467392 & 3.381791 & -0.111243 \\
\hline 14 & 6 & 10061002 & -5.599188 & 4.655193 & 0.291805 \\
\hline 15 & 6 & 10061000 & -4.512804 & 5.303237 & 1.043614 \\
\hline 16 & 6 & 10061000 & -3.344520 & 4.583551 & 1.292281 \\
\hline 17 & 6 & 10061002 & -2.297891 & 5.224624 & 2.130791 \\
\hline 18 & 6 & 10061000 & -2.478453 & 6.474073 & 2.596324 \\
\hline 19 & 6 & 10061000 & -3.645495 & 7.201712 & 2.314627 \\
\hline 20 & 6 & 10061002 & -4.639658 & 6.676216 & 1.577283 \\
\hline 21 & 6 & 10061000 & -1.029847 & 6.873982 & -0.489790 \\
\hline 22 & 6 & 10061000 & -2.053251 & 7.556917 & -1.150420 \\
\hline 23 & 6 & 10061000 & -2.902541 & 6.868748 & -2.019454 \\
\hline 24 & 6 & 10061000 & -2.724019 & 5.499281 & -2.234282 \\
\hline 25 & 6 & 10061000 & -1.699679 & 4.804288 & -1.576113 \\
\hline 26 & 6 & 10061000 & -0.857933 & 5.500977 & -0.691498 \\
\hline 27 & 15 & 10151003 & -1.527234 & 2.998288 & -1.904312 \\
\hline 28 & 6 & 10061000 & 0.047370 & 3.080901 & -2.861930 \\
\hline 29 & 6 & 10061000 & 1.253221 & 3.575958 & -2.333422 \\
\hline 30 & 6 & 10061000 & 2.375908 & 3.715524 & -3.154804 \\
\hline 31 & 6 & 10061000 & 2.320143 & 3.331984 & -4.495450 \\
\hline 32 & 6 & 10061000 & 1.129776 & 2.834432 & -5.026806 \\
\hline 33 & 6 & 10061000 & -0.005069 & 2.727910 & -4.218604 \\
\hline 34 & 15 & 10151003 & -4.289653 & 0.861584 & -0.375629 \\
\hline 35 & 6 & 10061000 & -5.623348 & 0.211410 & 0.695743 \\
\hline 36 & 6 & 10061000 & -5.265686 & -0.367844 & 1.919407 \\
\hline 37 & 6 & 10061000 & -6.245793 & -0.895232 & 2.764428 \\
\hline 38 & 6 & 10061000 & -7.591945 & -0.836261 & 2.394878 \\
\hline 39 & 6 & 10061000 & -7.958507 & -0.242122 & 1.183939 \\
\hline 40 & 6 & 10061000 & -6.979246 & 0.286004 & 0.336838 \\
\hline 41 & 6 & 10061000 & -4.941150 & 0.738355 & -2.082839 \\
\hline 42 & 6 & 10061000 & -4.820510 & 1.813628 & -2.976060 \\
\hline 43 & 6 & 10061000 & -5.354323 & 1.724938 & -4.264666 \\
\hline 44 & 6 & 10061000 & -6.023627 & 0.566731 & -4.666639 \\
\hline 45 & 6 & 10061000 & -6.149248 & -0.507837 & -3.783262 \\
\hline 46 & 6 & 10061000 & -5.592471 & -0.431406 & -2.502940 \\
\hline 47 & 1 & 10011000 & -4.225762 & -0.409914 & 2.215404 \\
\hline 48 & 1 & 10011000 & -5.962288 & -1.347795 & 3.705733 \\
\hline 49 & 1 & 10011000 & -8.351021 & -1.245449 & 3.048827 \\
\hline 50 & 1 & 10011000 & -9.002059 & -0.190539 & 0.901693 \\
\hline 51 & 1 & 10011000 & -7.284012 & 0.743980 & -0.593712 \\
\hline 52 & 1 & 10011000 & -5.691598 & -1.275419 & -1.834322 \\
\hline 53 & 1 & 10011000 & -6.668244 & -1.405068 & -4.094145 \\
\hline 54 & 1 & 10011000 & -6.444539 & 0.501873 & -5.661527 \\
\hline 55 & 1 & 10011000 & -5.258019 & 2.558759 & -4.947920 \\
\hline 56 & 1 & 10011000 & -4.327399 & 2.725390 & -2.677161 \\
\hline 57 & 1 & 10011000 & 1.341646 & 3.847006 & -1.294672 \\
\hline 58 & 1 & 10011000 & 3.287633 & 4.134007 & -2.756456 \\
\hline 59 & 1 & 10011000 & 3.194839 & 3.433602 & -5.124765 \\
\hline 60 & 1 & 10011000 & 1.079519 & 2.551871 & -6.070314 \\
\hline 61 & 1 & 10011000 & -0.933645 & 2.387108 & -4.657337 \\
\hline 62 & 1 & 10011000 & -6.299416 & 2.925563 & -0.629235 \\
\hline 63 & 1 & 10011000 & -6.520683 & 5.193891 & 0.092950 \\
\hline 64 & 1 & 10011000 & -5.534499 & 7.258390 & 1.379600 \\
\hline 65 & 1 & 10011000 & -3.747439 & 8.206885 & 2.703401 \\
\hline 66 & 1 & 10011000 & -1.703577 & 6.932767 & 3.197199 \\
\hline 67 & 1 & 10011000 & -1.377153 & 4.700269 & 2.363700 \\
\hline 68 & 1 & 10011000 & -3.407219 & 2.495132 & 3.318629 \\
\hline 69 & 1 & 10011000 & -2.736677 & 1.474758 & 5.388183 \\
\hline 70 & 1 & 10011000 & -0.643166 & 0.203143 & 5.574891 \\
\hline 71 & 1 & 10011000 & 0.794731 & -0.133704 & 3.662682 \\
\hline 72 & 1 & 10011000 & 1.342710 & 0.307808 & 1.193625 \\
\hline 73 & 1 & 10011000 & 0.836763 & 1.360832 & -0.899670 \\
\hline 74 & 1 & 10011000 & -2.193876 & 8.616562 & -0.981631 \\
\hline 75 & 1 & 10011000 & -0.385445 & 7.403250 & 0.200096 \\
\hline 76 & 1 & 10011000 & -0.103276 & 4.987094 & -0.117098 \\
\hline 77 & 1 & 10011000 & -3.385708 & 4.980509 & -2.915417 \\
\hline 78 & 1 & 10011000 & -3.699041 & 7.396380 & -2.527758 \\
\hline 79 & 79 & 10791004 & 3.120795 & 0.403785 & -0.465720 \\
\hline 80 & 79 & 10791004 & -2.379156 & -0.631733 & 0.154060 \\
\hline 81 & 6 & 10061000 & 0.583394 & -1.726686 & -1.952208 \\
\hline 82 & 6 & 10061002 & 0.119355 & -0.626901 & -2.835283 \\
\hline 83 & 6 & 10061002 & -1.068983 & -0.669886 & -3.451203 \\
\hline 84 & 6 & 10061000 & -1.956485 & -1.828528 & -3.278788 \\
\hline 85 & 6 & 10061002 & -3.169283 & -1.981798 & -4.110125 \\
\hline 86 & 6 & 10061000 & -3.933845 & -3.080333 & -3.990581 \\
\hline 87 & 6 & 10061000 & -3.611681 & -4.099804 & -3.085282 \\
\hline 88 & 6 & 10061002 & -2.517316 & -4.028939 & -2.308320 \\
\hline 89 & 6 & 10061000 & -1.615953 & -2.845353 & -2.382568 \\
\hline 90 & 6 & 10061000 & -0.327283 & -2.771642 & -1.636803 \\
\hline
\end{tabular}




\begin{tabular}{|c|c|c|c|c|c|}
\hline 91 & 6 & 10061002 & 0.012101 & -3.900540 & -0.747659 \\
\hline 92 & 6 & 10061002 & -0.477915 & -3.988098 & 0.520405 \\
\hline 93 & 6 & 10061002 & -0.129733 & -5.190088 & 1.336884 \\
\hline 94 & 6 & 10061000 & 0.564551 & -6.213289 & 0.819842 \\
\hline 95 & 6 & 10061000 & 0.977462 & -6.198807 & -0.512588 \\
\hline 96 & 6 & 10061000 & 0.707387 & -5.089549 & -1.327311 \\
\hline 97 & 6 & 10061002 & 1.109633 & -5.164713 & -2.756525 \\
\hline 98 & 6 & 10061000 & 1.763375 & -6.243186 & -3.221474 \\
\hline 99 & 6 & 10061000 & 2.068300 & -7.330850 & -2.390711 \\
\hline 100 & 6 & 10061002 & 1.706178 & -7.349101 & -1.096514 \\
\hline 101 & 6 & 10061000 & 4.128025 & -5.073931 & -0.024193 \\
\hline 102 & 6 & 10061000 & 3.879912 & -4.975110 & 1.346560 \\
\hline 103 & 6 & 10061000 & 3.160819 & -3.888315 & 1.848139 \\
\hline 104 & 6 & 10061000 & 2.679425 & -2.904749 & 0.978190 \\
\hline 105 & 6 & 10061000 & 2.895447 & -3.011913 & -0.401097 \\
\hline 106 & 6 & 10061000 & 3.647057 & -4.092720 & -0.896165 \\
\hline 107 & 15 & 10151003 & 2.374881 & -1.623664 & -1.474467 \\
\hline 108 & 6 & 10061000 & 3.332457 & -1.841321 & -3.023784 \\
\hline 109 & 6 & 10061000 & 4.723853 & -1.669720 & -2.978248 \\
\hline 110 & 6 & 10061000 & 5.493292 & -1.816590 & -4.135637 \\
\hline 111 & 6 & 10061000 & 4.878096 & -2.137368 & -5.347897 \\
\hline 112 & 6 & 10061000 & 3.492938 & -2.310776 & -5.402983 \\
\hline 113 & 6 & 10061000 & 2.720085 & -2.162223 & -4.247329 \\
\hline 114 & 15 & 10151003 & -1.713919 & -2.789039 & 1.209175 \\
\hline 115 & 6 & 10061000 & -3.272359 & -3.745292 & 1.086931 \\
\hline 116 & 6 & 10061000 & -4.306287 & -3.246791 & 0.283387 \\
\hline 117 & 6 & 10061000 & -5.519319 & -3.932292 & 0.183421 \\
\hline 118 & 6 & 10061000 & -5.709119 & -5.121455 & 0.891004 \\
\hline 119 & 6 & 10061000 & -4.686811 & -5.621705 & 1.702122 \\
\hline 120 & 6 & 10061000 & -3.472475 & -4.935571 & 1.804142 \\
\hline 121 & 6 & 10061000 & -1.463441 & -2.676353 & 3.023492 \\
\hline 122 & 6 & 10061000 & -2.558580 & -2.454966 & 3.873742 \\
\hline 123 & 6 & 10061000 & -2.382717 & -2.425565 & 5.260477 \\
\hline 124 & 6 & 10061000 & -1.110959 & -2.610845 & 5.808647 \\
\hline 125 & 6 & 10061000 & -0.012754 & -2.808777 & 4.968766 \\
\hline 126 & 6 & 10061000 & -0.187208 & -2.830831 & 3.582329 \\
\hline 127 & 1 & 10011000 & -4.169204 & -2.332680 & -0.275342 \\
\hline 128 & 1 & 10011000 & -6.310922 & -3.541440 & -0.442733 \\
\hline 129 & 1 & 10011000 & -6.649072 & -5.652429 & 0.814809 \\
\hline 130 & 1 & 10011000 & -4.838494 & -6.537260 & 2.258972 \\
\hline 131 & 1 & 10011000 & -2.709493 & -5.321923 & 2.461822 \\
\hline 132 & 1 & 10011000 & 0.669957 & -2.978977 & 2.949294 \\
\hline 133 & 1 & 10011000 & 0.973691 & -2.944979 & 5.390870 \\
\hline 134 & 1 & 10011000 & -0.974735 & -2.589619 & 6.882041 \\
\hline 135 & 1 & 10011000 & -3.233237 & -2.263551 & 5.909751 \\
\hline 136 & 1 & 10011000 & -3.552205 & -2.330472 & 3.468597 \\
\hline 137 & 1 & 10011000 & 5.212356 & -1.423780 & -2.043545 \\
\hline 138 & 1 & 10011000 & 6.566303 & -1.681978 & -4.092270 \\
\hline 139 & 1 & 10011000 & 5.474187 & -2.251904 & -6.243946 \\
\hline 140 & 1 & 10011000 & 3.017031 & -2.560161 & -6.342558 \\
\hline 141 & 1 & 10011000 & 1.653249 & -2.303696 & -4.321593 \\
\hline 142 & 1 & 10011000 & -0.439383 & -5.254824 & 2.372348 \\
\hline 143 & 1 & 10011000 & 0.791038 & -7.067503 & 1.446365 \\
\hline 144 & 1 & 10011000 & 1.951739 & -8.210307 & -0.482014 \\
\hline 145 & 1 & 10011000 & 2.599996 & -8.178048 & -2.804835 \\
\hline 146 & 1 & 10011000 & 2.058320 & -6.275847 & -4.262629 \\
\hline 147 & 1 & 10011000 & 0.877481 & -4.355929 & -3.437943 \\
\hline 148 & 1 & 10011000 & -2.303506 & -4.849403 & -1.631484 \\
\hline 149 & 1 & 10011000 & -4.256233 & -4.966889 & -3.015957 \\
\hline 150 & 1 & 10011000 & -4.817530 & -3.182504 & -4.607169 \\
\hline 151 & 1 & 10011000 & -3.438635 & -1.211262 & -4.826085 \\
\hline 152 & 1 & 10011000 & -1.360564 & 0.131279 & -4.119811 \\
\hline 153 & 1 & 10011000 & 0.780745 & 0.210173 & -3.031719 \\
\hline 154 & 1 & 10011000 & 4.250001 & -5.736789 & 2.020260 \\
\hline 155 & 1 & 10011000 & 4.689745 & -5.913446 & -0.412651 \\
\hline 156 & 1 & 10011000 & 3.850676 & -4.194768 & -1.952362 \\
\hline 157 & 1 & 10011000 & 2.151801 & -2.049345 & 1.378326 \\
\hline 158 & 1 & 10011000 & 2.984397 & -3.805059 & 2.912364 \\
\hline 159 & 6 & 10061003 & 4.009685 & 2.471787 & 1.745570 \\
\hline 160 & 6 & 10061003 & 4.840433 & 2.941960 & -0.399921 \\
\hline 161 & 6 & 10061000 & 5.303434 & 2.495537 & -1.782409 \\
\hline 162 & 8 & 10081002 & 5.056604 & 1.413912 & -2.343030 \\
\hline 163 & 6 & 10061000 & 3.705010 & 1.454686 & 2.835274 \\
\hline 164 & 6 & 10061000 & 4.157120 & 0.116584 & 2.773837 \\
\hline 165 & 6 & 10061000 & 3.041289 & 1.874980 & 4.001771 \\
\hline 166 & 6 & 10061000 & 3.936935 & -0.769452 & 3.834987 \\
\hline 167 & 1 & 10011000 & 4.697719 & -0.220741 & 1.893995 \\
\hline 168 & 6 & 10061000 & 2.831453 & 1.007005 & 5.090168 \\
\hline 169 & 1 & 10011000 & 2.698975 & 2.905840 & 4.079871 \\
\hline 170 & 6 & 10061000 & 3.273976 & -0.327912 & 4.999104 \\
\hline
\end{tabular}




\begin{tabular}{|c|c|c|c|c|c|}
\hline 171 & 1 & 10011000 & 4.296279 & -1.793661 & 3.802004 \\
\hline 172 & 1 & 10011000 & 2.331880 & 1.375410 & 5.980585 \\
\hline 173 & 7 & 10071003 & 4.115513 & 1.940638 & 0.378747 \\
\hline 174 & 8 & 10081003 & 3.096634 & -1.291787 & 5.996372 \\
\hline 175 & 8 & 10081000 & 6.001787 & 3.510001 & -2.388191 \\
\hline 176 & 6 & 10061003 & 2.490755 & -0.884479 & 7.256737 \\
\hline 177 & 1 & 10011000 & 2.486718 & -1.781611 & 7.878823 \\
\hline 178 & 1 & 10011000 & 3.080839 & -0.098036 & 7.745722 \\
\hline 179 & 1 & 10011000 & 1.459180 & -0.532804 & 7.108261 \\
\hline 180 & 6 & 10061003 & 6.645319 & 3.203139 & -3.679495 \\
\hline 181 & 1 & 10011000 & 7.391512 & 2.417613 & -3.529791 \\
\hline 182 & 1 & 10011000 & 5.896480 & 2.875295 & -4.407432 \\
\hline 183 & 1 & 10011000 & 7.114506 & 4.137556 & -3.988890 \\
\hline 184 & 1 & 10011000 & 3.239752 & 3.268039 & 1.809369 \\
\hline 185 & 1 & 10011000 & 4.255156 & 3.870901 & -0.565176 \\
\hline 186 & 6 & 10061003 & 6.015014 & 3.339321 & 0.542483 \\
\hline 187 & 6 & 10061003 & 5.405582 & 3.254696 & 1.961962 \\
\hline 188 & 1 & 10011000 & 6.432380 & 4.317263 & 0.283842 \\
\hline 189 & 1 & 10011000 & 5.216177 & 4.220639 & 2.436553 \\
\hline 190 & 7 & 10071000 & 7.364622 & 1.913851 & 1.885081 \\
\hline 191 & 6 & 10061003 & 8.450661 & 1.018635 & 2.297189 \\
\hline 192 & 1 & 10011000 & 8.425464 & 0.939890 & 3.386868 \\
\hline 193 & 1 & 10011000 & 8.318148 & 0.029164 & 1.846841 \\
\hline 194 & 1 & 10011000 & 9.416082 & 1.420846 & 1.973671 \\
\hline 195 & 6 & 10061002 & 6.442571 & 2.494827 & 2.783387 \\
\hline 196 & 8 & 10081002 & 6.508476 & 2.384741 & 4.019496 \\
\hline 197 & 6 & 10061002 & 7.146293 & 2.300798 & 0.558007 \\
\hline 198 & 8 & 10081002 & 7.796345 & 1.874278 & -0.415554 \\
\hline
\end{tabular}

\section{$(R, R, R, \mathbf{S})-\mathbf{I I}$}

\begin{tabular}{|c|c|c|c|c|c|}
\hline \multirow{2}{*}{$\begin{array}{l}\text { Center } \\
\text { Number }\end{array}$} & \multirow{2}{*}{$\begin{array}{l}\text { Atomic } \\
\text { Number }\end{array}$} & \multirow{2}{*}{$\begin{array}{c}\text { Atomic } \\
\text { Type }\end{array}$} & \multicolumn{3}{|c|}{ Coordinates (Angstroms) } \\
\hline & & & $\mathrm{x}$ & $\mathrm{Y}$ & $\mathrm{Z}$ \\
\hline 1 & 6 & 10061002 & 1.792542 & -2.170521 & 0.223949 \\
\hline 2 & 6 & 10061002 & 0.607857 & -1.533441 & 0.872692 \\
\hline 3 & 6 & 10061002 & 0.676703 & -1.029932 & 2.113630 \\
\hline 4 & 6 & 10061000 & 1.947966 & -1.055442 & 2.851146 \\
\hline 5 & 6 & 10061002 & 2.035101 & -0.592680 & 4.251938 \\
\hline 6 & 6 & 10061000 & 3.227240 & -0.544905 & 4.870705 \\
\hline 7 & 6 & 10061000 & 4.407509 & -0.924129 & 4.214976 \\
\hline 8 & 6 & 10061002 & 4.392775 & -1.383555 & 2.951301 \\
\hline 9 & 6 & 10061000 & 3.106332 & -1.502579 & 2.213521 \\
\hline 10 & 6 & 10061002 & 3.010767 & -2.084563 & 0.842724 \\
\hline 11 & 6 & 10061002 & 4.253924 & -2.578284 & 0.192968 \\
\hline 12 & 6 & 10061000 & 4.871584 & -1.869285 & -0.791073 \\
\hline 13 & 6 & 10061000 & 5.974595 & -2.414361 & -1.483631 \\
\hline 14 & 6 & 10061002 & 6.489714 & -3.615904 & -1.181242 \\
\hline 15 & 6 & 10061000 & 5.941452 & -4.386566 & -0.054514 \\
\hline 16 & 6 & 10061000 & 4.852519 & -3.870113 & 0.647439 \\
\hline 17 & 6 & 10061002 & 4.359406 & -4.634084 & 1.824394 \\
\hline 18 & 6 & 10061000 & 4.944794 & -5.795687 & 2.168330 \\
\hline 19 & 6 & 10061000 & 6.026024 & -6.317447 & 1.440945 \\
\hline 20 & 6 & 10061002 & 6.529893 & -5.673618 & 0.373462 \\
\hline 21 & 6 & 10061000 & 2.823652 & -6.582053 & -0.450090 \\
\hline 22 & 6 & 10061000 & 3.638107 & -6.949481 & -1.522870 \\
\hline 23 & 6 & 10061000 & 3.851860 & -6.054892 & -2.573225 \\
\hline 24 & 6 & 10061000 & 3.246526 & -4.795222 & -2.554232 \\
\hline 25 & 6 & 10061000 & 2.433285 & -4.411926 & -1.477856 \\
\hline 26 & 6 & 10061000 & 2.228436 & -5.316983 & -0.421506 \\
\hline 27 & 15 & 10151003 & 1.625561 & -2.751323 & -1.543678 \\
\hline 28 & 6 & 10061000 & -0.099692 & -3.405735 & -1.631590 \\
\hline 29 & 6 & 10061000 & -0.738816 & -4.044308 & -0.551967 \\
\hline 30 & 6 & 10061000 & -1.961185 & -4.694468 & -0.740282 \\
\hline 31 & 6 & 10061000 & -2.568772 & -4.695809 & -1.996619 \\
\hline 32 & 6 & 10061000 & -0.729889 & -3.397052 & -2.884552 \\
\hline 33 & 15 & 10151003 & 4.404592 & -0.126083 & -1.149455 \\
\hline 34 & 6 & 10061000 & 5.833154 & 0.759107 & -0.424232 \\
\hline 35 & 6 & 10061000 & 5.662636 & 1.401598 & 0.808290 \\
\hline 36 & 6 & 10061000 & 6.729311 & 2.078632 & 1.405553 \\
\hline 37 & 6 & 10061000 & 7.976818 & 2.109213 & 0.777209 \\
\hline 38 & 6 & 10061000 & 8.159174 & 1.456823 & -0.445333 \\
\hline 39 & 6 & 10061000 & 7.093455 & 0.777465 & -1.043550 \\
\hline 40 & 6 & 10061000 & 4.534535 & 0.138781 & -2.955040 \\
\hline 41 & 6 & 10061000 & 4.143331 & -0.872563 & -3.842850 \\
\hline 42 & 6 & 10061000 & 4.283320 & -0.694720 & -5.222132 \\
\hline
\end{tabular}




\begin{tabular}{|c|c|c|c|c|c|}
\hline 43 & 6 & 10061000 & 4.809885 & 0.498529 & -5.723506 \\
\hline 44 & 6 & 10061000 & 5.181902 & 1.520015 & -4.845700 \\
\hline 45 & 6 & 10061000 & 5.027741 & 1.349496 & -3.466588 \\
\hline 46 & 1 & 10011000 & 4.704002 & 1.369623 & 1.308234 \\
\hline 47 & 1 & 10011000 & 6.590863 & 2.575447 & 2.356887 \\
\hline 48 & 1 & 10011000 & 8.802896 & 2.633821 & 1.239465 \\
\hline 49 & 1 & 10011000 & 9.126811 & 1.477758 & -0.929743 \\
\hline 50 & 1 & 10011000 & 7.252813 & 0.285523 & -1.992186 \\
\hline 51 & 1 & 10011000 & 5.304136 & 2.157285 & -2.802705 \\
\hline 52 & 1 & 10011000 & 5.576704 & 2.449787 & -5.234230 \\
\hline 53 & 1 & 10011000 & 4.920393 & 0.635391 & -6.791300 \\
\hline 54 & 1 & 10011000 & 3.985794 & -1.482683 & -5.901673 \\
\hline 55 & 1 & 10011000 & 3.740909 & -1.800610 & -3.468254 \\
\hline 56 & 1 & 10011000 & -0.300157 & -4.057764 & 0.432954 \\
\hline 57 & 1 & 10011000 & -2.434521 & -5.204567 & 0.087977 \\
\hline 58 & 1 & 10011000 & -3.517222 & -5.197555 & -2.138607 \\
\hline 59 & 1 & 10011000 & -0.250184 & -2.924214 & -3.732160 \\
\hline 60 & 1 & 10011000 & 6.431759 & -1.858084 & -2.289389 \\
\hline 61 & 1 & 10011000 & 7.335365 & -4.000097 & -1.743469 \\
\hline 62 & 1 & 10011000 & 7.368654 & -6.097960 & -0.169728 \\
\hline 63 & 1 & 10011000 & 6.463460 & -7.258844 & 1.747993 \\
\hline 64 & 1 & 10011000 & 4.569178 & -6.346009 & 3.021558 \\
\hline 65 & 1 & 10011000 & 3.516844 & -4.274961 & 2.405245 \\
\hline 66 & 1 & 10011000 & 5.329600 & -1.663821 & 2.481094 \\
\hline 67 & 1 & 10011000 & 5.350387 & -0.850107 & 4.741742 \\
\hline 68 & 1 & 10011000 & 3.280364 & -0.198577 & 5.894926 \\
\hline 69 & 1 & 10011000 & 1.136640 & -0.294777 & 4.783024 \\
\hline 70 & 1 & 10011000 & -0.199945 & -0.581099 & 2.566614 \\
\hline 71 & 1 & 10011000 & -0.331445 & -1.459955 & 0.336515 \\
\hline 72 & 1 & 10011000 & 4.108354 & -7.924048 & -1.536882 \\
\hline 73 & 1 & 10011000 & 2.671617 & -7.269125 & 0.372160 \\
\hline 74 & 1 & 10011000 & 1.654789 & -5.039756 & 0.447176 \\
\hline 75 & 1 & 10011000 & 3.406290 & -4.122968 & -3.385594 \\
\hline 76 & 1 & 10011000 & 4.482684 & -6.339060 & -3.405397 \\
\hline 77 & 79 & 10791004 & -3.332023 & -1.101872 & 0.400493 \\
\hline 78 & 79 & 10791004 & 2.453907 & 0.946597 & -0.085576 \\
\hline 79 & 6 & 10061000 & -1.192540 & 1.369714 & -1.139242 \\
\hline 80 & 6 & 10061002 & -0.789578 & 0.262134 & -2.040710 \\
\hline 81 & 6 & 10061002 & 0.174669 & 0.417126 & -2.957810 \\
\hline 82 & 6 & 10061000 & 0.854486 & 1.714580 & -3.109939 \\
\hline 83 & 6 & 10061002 & 1.754200 & 1.969919 & -4.254887 \\
\hline 84 & 6 & 10061000 & 2.304711 & 3.184643 & -4.421531 \\
\hline 85 & 6 & 10061000 & 2.057718 & 4.229796 & -3.521504 \\
\hline 86 & 6 & 10061002 & 1.245020 & 4.067903 & -2.463463 \\
\hline 87 & 6 & 10061000 & 0.586654 & 2.756619 & -2.215876 \\
\hline 88 & 6 & 10061000 & -0.425838 & 2.564020 & -1.142118 \\
\hline 89 & 6 & 10061002 & -0.665150 & 3.673488 & -0.195046 \\
\hline 90 & 6 & 10061002 & 0.113708 & 3.853847 & 0.906567 \\
\hline 91 & 6 & 10061002 & -0.227813 & 4.961339 & 1.848237 \\
\hline 92 & 6 & 10061000 & -1.196989 & 5.844336 & 1.568575 \\
\hline 93 & 6 & 10061000 & -1.912898 & 5.767256 & 0.372818 \\
\hline 94 & 6 & 10061000 & -1.664458 & 4.728742 & -0.537411 \\
\hline 95 & 6 & 10061002 & -2.389893 & 4.755001 & -1.832577 \\
\hline 96 & 6 & 10061000 & -3.316807 & 5.698235 & -2.072476 \\
\hline 97 & 6 & 10061000 & -3.606962 & 6.697074 & -1.131859 \\
\hline 98 & 6 & 10061002 & -2.953603 & 6.768221 & 0.040766 \\
\hline 99 & 6 & 10061000 & -4.261352 & 4.140697 & 2.127221 \\
\hline 100 & 6 & 10061000 & -3.663250 & 3.841657 & 3.352609 \\
\hline 101 & 6 & 10061000 & -2.792046 & 2.755751 & 3.455139 \\
\hline 102 & 6 & 10061000 & -2.506551 & 1.976703 & 2.329081 \\
\hline 103 & 6 & 10061000 & -3.083963 & 2.281080 & 1.090974 \\
\hline 104 & 6 & 10061000 & -3.982583 & 3.359241 & 1.002049 \\
\hline 105 & 15 & 10151003 & -2.825082 & 1.116615 & -0.298921 \\
\hline 106 & 6 & 10061000 & -4.116432 & 1.507309 & -1.540593 \\
\hline 107 & 6 & 10061000 & -5.464642 & 1.415823 & -1.160940 \\
\hline 108 & 6 & 10061000 & -6.478957 & 1.700829 & -2.078657 \\
\hline 109 & 6 & 10061000 & -6.155427 & 2.046260 & -3.392615 \\
\hline 110 & 6 & 10061000 & -4.817706 & 2.094907 & -3.791750 \\
\hline 111 & 6 & 10061000 & -3.800458 & 1.817461 & -2.873512 \\
\hline 112 & 15 & 10151003 & 1.708393 & 2.947653 & 1.166379 \\
\hline 113 & 6 & 10061000 & 2.932214 & 4.201978 & 0.629703 \\
\hline 114 & 6 & 10061000 & 3.777233 & 3.905185 & -0.448514 \\
\hline 115 & 6 & 10061000 & 4.732377 & 4.831238 & -0.874842 \\
\hline 116 & 6 & 10061000 & 4.851790 & 6.061208 & -0.223788 \\
\hline 117 & 6 & 10061000 & 4.017213 & 6.363547 & 0.855612 \\
\hline 118 & 6 & 10061000 & 3.060810 & 5.437709 & 1.283846 \\
\hline 119 & 6 & 10061000 & 2.007628 & 2.824976 & 2.971025 \\
\hline 120 & 6 & 10061000 & 3.302308 & 2.971311 & 3.495149 \\
\hline 121 & 6 & 10061000 & 3.514355 & 2.923303 & 4.876143 \\
\hline 122 & 6 & 10061000 & 2.437165 & 2.722211 & 5.742984 \\
\hline
\end{tabular}




\begin{tabular}{|c|c|c|c|c|c|}
\hline 123 & 6 & 10061000 & 1.150256 & 2.551180 & 5.227163 \\
\hline 124 & 6 & 10061000 & 0.940768 & 2.587407 & 3.845678 \\
\hline 125 & 1 & 10011000 & 3.689703 & 2.961717 & -0.968462 \\
\hline 126 & 1 & 10011000 & 5.378335 & 4.595578 & -1.710637 \\
\hline 127 & 1 & 10011000 & 5.592380 & 6.778294 & -0.553278 \\
\hline 128 & 1 & 10011000 & 4.116023 & 7.313225 & 1.365217 \\
\hline 129 & 1 & 10011000 & 2.445777 & 5.679686 & 2.137036 \\
\hline 130 & 1 & 10011000 & -0.051397 & 2.441504 & 3.457261 \\
\hline 131 & 1 & 10011000 & 0.316848 & 2.379706 & 5.895898 \\
\hline 132 & 1 & 10011000 & 2.601484 & 2.686372 & 6.812000 \\
\hline 133 & 1 & 10011000 & 4.513436 & 3.044813 & 5.274059 \\
\hline 134 & 1 & 10011000 & 4.145970 & 3.142180 & 2.842558 \\
\hline 135 & 1 & 10011000 & -5.728551 & 1.135922 & -0.148895 \\
\hline 136 & 1 & 10011000 & -7.515877 & 1.641698 & -1.774390 \\
\hline 137 & 1 & 10011000 & -6.941303 & 2.259649 & -4.105359 \\
\hline 138 & 1 & 10011000 & -4.568440 & 2.343239 & -4.815200 \\
\hline 139 & 1 & 10011000 & -2.776756 & 1.843345 & -3.214814 \\
\hline 140 & 1 & 10011000 & 0.313896 & 5.069581 & 2.780313 \\
\hline 141 & 1 & 10011000 & -1.409434 & 6.636965 & 2.275695 \\
\hline 142 & 1 & 10011000 & -3.193582 & 7.561647 & 0.742520 \\
\hline 143 & 1 & 10011000 & -4.363766 & 7.435755 & -1.363320 \\
\hline 144 & 1 & 10011000 & -3.844065 & 5.694570 & -3.018087 \\
\hline 145 & 1 & 10011000 & -2.163442 & 4.029978 & -2.603159 \\
\hline 146 & 1 & 10011000 & 1.073966 & 4.908823 & -1.799402 \\
\hline 147 & 1 & 10011000 & 2.527398 & 5.191007 & -3.687433 \\
\hline 148 & 1 & 10011000 & 2.952508 & 3.362629 & -5.270092 \\
\hline 149 & 1 & 10011000 & 1.955292 & 1.182549 & -4.974077 \\
\hline 150 & 1 & 10011000 & 0.423741 & -0.405397 & -3.621076 \\
\hline 151 & 1 & 10011000 & -1.316475 & -0.684982 & -1.985881 \\
\hline 152 & 1 & 10011000 & -3.881851 & 4.445073 & 4.223843 \\
\hline 153 & 1 & 10011000 & -4.940730 & 4.979318 & 2.047604 \\
\hline 154 & 1 & 10011000 & -4.456389 & 3.616487 & 0.066343 \\
\hline 155 & 1 & 10011000 & -1.856624 & 1.118732 & 2.427288 \\
\hline 156 & 1 & 10011000 & -2.347529 & 2.509859 & 4.410719 \\
\hline 157 & 6 & 10061000 & -5.647391 & -3.042851 & 0.397091 \\
\hline 158 & 1 & 10011000 & -5.868883 & -4.124133 & 0.287956 \\
\hline 159 & 6 & 10061000 & -4.108773 & -3.603175 & 2.004915 \\
\hline 160 & 6 & 10061000 & -2.774470 & -3.469699 & 2.711938 \\
\hline 161 & 8 & 10081000 & -1.875056 & -2.652456 & 2.454785 \\
\hline 162 & 6 & 10061000 & -6.105856 & -2.381094 & -0.896612 \\
\hline 163 & 6 & 10061000 & -5.206235 & -2.237162 & -1.980081 \\
\hline 164 & 6 & 10061000 & -7.449572 & -2.019276 & -1.112069 \\
\hline 165 & 6 & 10061000 & -5.622297 & -1.734152 & -3.218287 \\
\hline 166 & 1 & 10011000 & -4.173567 & -2.533088 & -1.847222 \\
\hline 167 & 6 & 10061000 & -7.888201 & -1.516500 & -2.351664 \\
\hline 168 & 1 & 10011000 & -8.172278 & -2.095821 & -0.307088 \\
\hline 169 & 6 & 10061000 & -6.970476 & -1.369737 & -3.410411 \\
\hline 170 & 1 & 10011000 & -4.929864 & -1.629137 & -4.048629 \\
\hline 171 & 1 & 10011000 & -8.930265 & -1.237633 & -2.469219 \\
\hline 172 & 7 & 10071000 & -4.200317 & -2.935651 & 0.705328 \\
\hline 173 & 1 & 10011000 & -4.289502 & -4.685962 & 1.893926 \\
\hline 174 & 8 & 10081003 & -7.287310 & -0.868185 & -4.675228 \\
\hline 175 & 8 & 10081003 & -2.669752 & -4.406373 & 3.710804 \\
\hline 176 & 6 & 10061003 & -8.661804 & -0.462129 & -4.938146 \\
\hline 177 & 1 & 10011000 & -8.664473 & -0.102504 & -5.968884 \\
\hline 178 & 1 & 10011000 & -9.352933 & -1.310427 & -4.840806 \\
\hline 179 & 1 & 10011000 & -8.973305 & 0.346700 & -4.262430 \\
\hline 180 & 6 & 10061003 & -1.504321 & -4.300129 & 4.607220 \\
\hline 181 & 1 & 10011000 & -1.620101 & -5.119861 & 5.316902 \\
\hline 182 & 1 & 10011000 & -0.576457 & -4.401645 & 4.034885 \\
\hline 183 & 1 & 10011000 & -1.525641 & -3.331588 & 5.115778 \\
\hline 184 & 6 & 10061002 & -6.387006 & -2.665966 & 1.770134 \\
\hline 185 & 6 & 10061002 & -5.334326 & -3.049855 & 2.852843 \\
\hline 186 & 1 & 10011000 & -7.328026 & -3.218373 & 1.856758 \\
\hline 187 & 1 & 10011000 & -5.673150 & -3.791252 & 3.582016 \\
\hline 188 & 7 & 10071003 & -5.819866 & -0.731856 & 3.051341 \\
\hline 189 & 6 & 10061002 & -5.011719 & -1.744798 & 3.582471 \\
\hline 190 & 8 & 10081002 & -4.179542 & -1.575031 & 4.495929 \\
\hline 191 & 6 & 10061003 & -5.831581 & 0.647263 & 3.543428 \\
\hline 192 & 1 & 10011000 & -6.777843 & 0.866563 & 4.049749 \\
\hline 193 & 1 & 10011000 & -5.003487 & 0.761416 & 4.247544 \\
\hline 194 & 1 & 10011000 & -5.724778 & 1.343272 & 2.705359 \\
\hline 195 & 6 & 10061000 & -1.961789 & -4.032228 & -3.064735 \\
\hline 196 & 1 & 10011000 & -2.437150 & -4.025145 & -4.036979 \\
\hline 197 & 6 & 10061002 & -6.671942 & -1.184552 & 2.023943 \\
\hline 198 & 8 & 10081002 & -7.519682 & -0.450239 & 1.483395 \\
\hline
\end{tabular}


TS1

\begin{tabular}{|c|c|c|c|c|c|}
\hline \multirow{2}{*}{$\begin{array}{l}\text { Center } \\
\text { Number }\end{array}$} & \multirow{2}{*}{$\begin{array}{l}\text { Atomic } \\
\text { Number }\end{array}$} & \multirow{2}{*}{$\begin{array}{l}\text { Atomic } \\
\text { Type }\end{array}$} & \multicolumn{3}{|c|}{ Coordinates (Angstroms) } \\
\hline & & & $\mathrm{X}$ & $\mathrm{Y}$ & $\mathrm{z}$ \\
\hline & & & & & 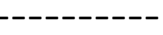 \\
\hline 1 & 6 & 10061000 & 1.481585 & -2.253307 & -0.021667 \\
\hline 2 & 6 & 10061002 & 0.200398 & -1.599027 & 0.359419 \\
\hline 3 & 6 & 10061002 & 0.032568 & -1.031778 & 1.563017 \\
\hline 4 & 6 & 10061000 & 1.129108 & -1.027116 & 2.549160 \\
\hline 5 & 6 & 10061002 & 0.945148 & -0.449061 & 3.895374 \\
\hline 6 & 6 & 10061000 & 1.959638 & -0.442720 & 4.775758 \\
\hline 7 & 6 & 10061000 & 3.222073 & -0.947765 & 4.438209 \\
\hline 8 & 6 & 10061002 & 3.463648 & -1.478273 & 3.227389 \\
\hline 9 & 6 & 10061000 & 2.376117 & -1.558644 & 2.214550 \\
\hline 10 & 6 & 10061000 & 2.575240 & -2.164461 & 0.874817 \\
\hline 11 & 6 & 10061002 & 3.925845 & -2.639178 & 0.498032 \\
\hline 12 & 6 & 10061000 & 4.743335 & -1.893185 & -0.292928 \\
\hline 13 & 6 & 10061000 & 5.972588 & -2.418933 & -0.747839 \\
\hline 14 & 6 & 10061002 & 6.410004 & -3.636149 & -0.389259 \\
\hline 15 & 6 & 10061000 & 5.628210 & -4.447092 & 0.557233 \\
\hline 16 & 6 & 10061000 & 4.410773 & -3.950697 & 1.021512 \\
\hline 17 & 6 & 10061002 & 3.676180 & -4.748739 & 2.038383 \\
\hline 18 & 6 & 10061000 & 4.174949 & -5.924565 & 2.461556 \\
\hline 19 & 6 & 10061000 & 5.388784 & -6.428320 & 1.967714 \\
\hline 20 & 6 & 10061002 & 6.111524 & -5.751647 & 1.057687 \\
\hline 21 & 6 & 10061000 & 2.651174 & -6.634553 & -0.552738 \\
\hline 22 & 6 & 10061000 & 3.688648 & -6.978044 & -1.421707 \\
\hline 23 & 6 & 10061000 & 4.133654 & -6.058155 & -2.373122 \\
\hline 24 & 6 & 10061000 & 3.536934 & -4.797288 & -2.460445 \\
\hline 25 & 6 & 10061000 & 2.498443 & -4.439156 & -1.588686 \\
\hline 26 & 6 & 10061000 & 2.062043 & -5.368556 & -0.628311 \\
\hline 27 & 15 & 10151003 & 1.727784 & -2.773804 & -1.792285 \\
\hline 28 & 6 & 10061000 & 0.066426 & -3.402088 & -2.295625 \\
\hline 29 & 6 & 10061000 & -0.805740 & -4.067536 & -1.414236 \\
\hline 30 & 6 & 10061000 & -1.970617 & -4.668478 & -1.896629 \\
\hline 31 & 6 & 10061000 & -2.295714 & -4.582605 & -3.251891 \\
\hline 32 & 6 & 10061000 & -1.454439 & -3.894898 & -4.127865 \\
\hline 33 & 6 & 10061000 & -0.273148 & -3.318033 & -3.653931 \\
\hline 34 & 15 & 10151003 & 4.357093 & -0.134720 & -0.682946 \\
\hline 35 & 6 & 10061000 & 5.608267 & 0.723342 & 0.341753 \\
\hline 36 & 6 & 10061000 & 5.187317 & 1.337654 & 1.527497 \\
\hline 37 & 6 & 10061000 & 6.106779 & 1.999534 & 2.345577 \\
\hline 38 & 6 & 10061000 & 7.456177 & 2.043627 & 1.986144 \\
\hline 39 & 6 & 10061000 & 7.886675 & 1.420757 & 0.811313 \\
\hline 40 & 6 & 10061000 & 6.968114 & 0.757058 & -0.007834 \\
\hline 41 & 6 & 10061000 & 4.857981 & 0.175485 & -2.415371 \\
\hline 42 & 6 & 10061000 & 4.675465 & -0.817756 & -3.387508 \\
\hline 43 & 6 & 10061000 & 5.110174 & -0.610418 & -4.699570 \\
\hline 44 & 6 & 10061000 & 5.725875 & 0.593863 & -5.050191 \\
\hline 45 & 6 & 10061000 & 5.892364 & 1.597071 & -4.091916 \\
\hline 46 & 6 & 10061000 & 5.443053 & 1.397397 & -2.783108 \\
\hline 47 & 1 & 10011000 & 4.146787 & 1.295614 & 1.819342 \\
\hline 48 & 1 & 10011000 & 5.774503 & 2.474586 & 3.259378 \\
\hline 49 & 1 & 10011000 & 8.168401 & 2.556551 & 2.619288 \\
\hline 50 & 1 & 10011000 & 8.932454 & 1.453367 & 0.534503 \\
\hline 51 & 1 & 10011000 & 7.319545 & 0.291265 & -0.917010 \\
\hline 52 & 1 & 10011000 & 5.567614 & 2.189682 & -2.057938 \\
\hline 53 & 1 & 10011000 & 6.357483 & 2.535000 & -4.366155 \\
\hline 54 & 1 & 10011000 & 6.065289 & 0.753144 & -6.065369 \\
\hline 55 & 1 & 10011000 & 4.972929 & -1.384750 & -5.443087 \\
\hline 56 & 1 & 10011000 & 4.210454 & -1.756054 & -3.128077 \\
\hline 57 & 1 & 10011000 & -0.596661 & -4.132990 & -0.358388 \\
\hline 58 & 1 & 10011000 & -2.621978 & -5.202864 & -1.217841 \\
\hline 59 & 1 & 10011000 & -3.199290 & -5.048374 & -3.622193 \\
\hline 60 & 1 & 10011000 & -1.705817 & -3.828064 & -5.178390 \\
\hline 61 & 1 & 10011000 & 0.391230 & -2.825487 & -4.352365 \\
\hline 62 & 1 & 10011000 & 6.594158 & -1.835605 & -1.411615 \\
\hline 63 & 1 & 10011000 & 7.358996 & -4.004163 & -0.767234 \\
\hline 64 & 1 & 10011000 & 7.049254 & -6.161891 & 0.695681 \\
\hline 65 & 1 & 10011000 & 5.750810 & -7.381934 & 2.330182 \\
\hline 66 & 1 & 10011000 & 3.625128 & -6.500016 & 3.195353 \\
\hline 67 & 1 & 10011000 & 2.729460 & -4.400276 & 2.437369 \\
\hline 68 & 1 & 10011000 & 4.459213 & -1.846942 & 3.003995 \\
\hline 69 & 1 & 10011000 & 4.020553 & -0.903298 & 5.167875 \\
\hline 70 & 1 & 10011000 & 1.805519 & -0.021623 & 5.760856 \\
\hline 71 & 1 & 10011000 & -0.004789 & -0.015713 & 4.168933 \\
\hline 72 & 1 & 10011000 & -0.910264 & -0.551215 & 1.801234 \\
\hline
\end{tabular}




\begin{tabular}{|c|c|c|c|c|c|}
\hline 73 & 1 & 10011000 & -0.614335 & -1.546827 & -0.351840 \\
\hline 74 & 1 & 10011000 & 4.152262 & -7.953475 & -1.352719 \\
\hline 75 & 1 & 10011000 & 2.318406 & -7.341182 & 0.196437 \\
\hline 76 & 1 & 10011000 & 1.305877 & -5.110719 & 0.094374 \\
\hline 77 & 1 & 10011000 & 3.881369 & -4.103288 & -3.214790 \\
\hline 78 & 1 & 10011000 & 4.938387 & -6.323221 & -3.046411 \\
\hline 79 & 79 & 10791004 & -3.204808 & -1.013608 & -0.113524 \\
\hline 80 & 79 & 10791004 & 2.237662 & 0.954715 & -0.005028 \\
\hline 81 & 6 & 10061000 & -1.066946 & 1.333234 & -1.836997 \\
\hline 82 & 6 & 10061002 & -0.486022 & 0.238486 & -2.653497 \\
\hline 83 & 6 & 10061002 & 0.635879 & 0.408832 & -3.365054 \\
\hline 84 & 6 & 10061000 & 1.327311 & 1.707707 & -3.363016 \\
\hline 85 & 6 & 10061002 & 2.426092 & 1.982387 & -4.313206 \\
\hline 86 & 6 & 10061000 & 2.995306 & 3.199087 & -4.351800 \\
\hline 87 & 6 & 10061000 & 2.581902 & 4.227530 & -3.494998 \\
\hline 88 & 6 & 10061002 & 1.583548 & 4.048104 & -2.613539 \\
\hline 89 & 6 & 10061000 & 0.889626 & 2.734109 & -2.519905 \\
\hline 90 & 6 & 10061000 & -0.316121 & 2.526901 & -1.669681 \\
\hline 91 & 6 & 10061002 & -0.769412 & 3.652469 & -0.827688 \\
\hline 92 & 6 & 10061002 & -0.229222 & 3.894441 & 0.398689 \\
\hline 93 & 6 & 10061002 & -0.737895 & 5.058234 & 1.184421 \\
\hline 94 & 6 & 10061000 & -1.622163 & 5.921918 & 0.665457 \\
\hline 95 & 6 & 10061000 & -2.094164 & 5.767855 & -0.638800 \\
\hline 96 & 6 & 10061000 & -1.685491 & 4.674692 & -1.417046 \\
\hline 97 & 6 & 10061002 & -2.173264 & 4.599757 & -2.819164 \\
\hline 98 & 6 & 10061000 & -3.023667 & 5.527233 & -3.291690 \\
\hline 99 & 6 & 10061000 & -3.460916 & 6.595413 & -2.495310 \\
\hline 100 & 6 & 10061002 & -3.036801 & 6.746912 & -1.228835 \\
\hline 101 & 6 & 10061000 & -5.006993 & 4.219543 & 0.141832 \\
\hline 102 & 6 & 10061000 & -4.673744 & 4.226971 & 1.498434 \\
\hline 103 & 6 & 10061000 & -3.756675 & 3.298587 & 1.996248 \\
\hline 104 & 6 & 10061000 & -3.164034 & 2.370315 & 1.135197 \\
\hline 105 & 6 & 10061000 & -3.469150 & 2.374311 & -0.229960 \\
\hline 106 & 6 & 10061000 & -4.415900 & 3.290865 & -0.720198 \\
\hline 107 & 15 & 10151003 & -2.801916 & 1.023931 & -1.263564 \\
\hline 108 & 6 & 10061000 & -3.852259 & 0.995790 & -2.764492 \\
\hline 109 & 6 & 10061000 & -5.200386 & 0.635439 & -2.629179 \\
\hline 110 & 6 & 10061000 & -6.034641 & 0.580506 & -3.748605 \\
\hline 111 & 6 & 10061000 & -5.527435 & 0.888299 & -5.013178 \\
\hline 112 & 6 & 10061000 & -4.185527 & 1.250148 & -5.157774 \\
\hline 113 & 6 & 10061000 & -3.347721 & 1.302157 & -4.039434 \\
\hline 114 & 15 & 10151003 & 1.244301 & 2.976851 & 1.047725 \\
\hline 115 & 6 & 10061000 & 2.580401 & 4.206993 & 0.801660 \\
\hline 116 & 6 & 10061000 & 3.624049 & 3.903062 & -0.083187 \\
\hline 117 & 6 & 10061000 & 4.669327 & 4.808374 & -0.282407 \\
\hline 118 & 6 & 10061000 & 4.681445 & 6.023514 & 0.406141 \\
\hline 119 & 6 & 10061000 & 3.649474 & 6.331417 & 1.296686 \\
\hline 120 & 6 & 10061000 & 2.602481 & 5.426539 & 1.497242 \\
\hline 121 & 6 & 10061000 & 1.110499 & 2.884202 & 2.875506 \\
\hline 122 & 6 & 10061000 & 2.266508 & 2.922836 & 3.671592 \\
\hline 123 & 6 & 10061000 & 2.165853 & 2.902934 & 5.065926 \\
\hline 124 & 6 & 10061000 & 0.910976 & 2.832230 & 5.676183 \\
\hline 125 & 6 & 10061000 & -0.242908 & 2.777853 & 4.891489 \\
\hline 126 & 6 & 10061000 & -0.142428 & 2.794653 & 3.497506 \\
\hline 127 & 1 & 10011000 & 3.623887 & 2.969875 & -0.628494 \\
\hline 128 & 1 & 10011000 & 5.469032 & 4.568075 & -0.970811 \\
\hline 129 & 1 & 10011000 & 5.491870 & 6.724249 & 0.253101 \\
\hline 130 & 1 & 10011000 & 3.664715 & 7.268584 & 1.837837 \\
\hline 131 & 1 & 10011000 & 1.832778 & 5.670732 & 2.213000 \\
\hline 132 & 1 & 10011000 & -1.040733 & 2.752914 & 2.908201 \\
\hline 133 & 1 & 10011000 & -1.215367 & 2.721003 & 5.362513 \\
\hline 134 & 1 & 10011000 & 0.833211 & 2.813458 & 6.755425 \\
\hline 135 & 1 & 10011000 & 3.061062 & 2.940077 & 5.672888 \\
\hline 136 & 1 & 10011000 & 3.244479 & 2.991646 & 3.218238 \\
\hline 137 & 1 & 10011000 & -5.602769 & 0.397046 & -1.654106 \\
\hline 138 & 1 & 10011000 & -7.073507 & 0.298876 & -3.635245 \\
\hline 139 & 1 & 10011000 & -6.173382 & 0.846073 & -5.880506 \\
\hline 140 & 1 & 10011000 & -3.793252 & 1.488630 & -6.137916 \\
\hline 141 & 1 & 10011000 & -2.315607 & 1.583053 & -4.181760 \\
\hline 142 & 1 & 10011000 & -0.395282 & 5.221951 & 2.198625 \\
\hline 143 & 1 & 10011000 & -1.960429 & 6.756589 & 1.267375 \\
\hline 144 & 1 & 10011000 & -3.390167 & 7.589082 & -0.641086 \\
\hline 145 & 1 & 10011000 & -4.149555 & 7.318100 & -2.914209 \\
\hline 146 & 1 & 10011000 & -3.377059 & 5.451037 & -4.312180 \\
\hline 147 & 1 & 10011000 & -1.846447 & 3.802159 & -3.474684 \\
\hline 148 & 1 & 10011000 & 1.289946 & 4.878038 & -1.979362 \\
\hline 149 & 1 & 10011000 & 3.073386 & 5.190539 & -3.551167 \\
\hline 150 & 1 & 10011000 & 3.791116 & 3.391271 & -5.059817 \\
\hline 151 & 1 & 10011000 & 2.760656 & 1.207922 & -4.995783 \\
\hline 152 & 1 & 10011000 & 1.012404 & -0.402254 & -3.980004 \\
\hline
\end{tabular}




\begin{tabular}{|c|c|c|c|c|c|}
\hline 153 & 1 & 10011000 & -1.009760 & -0.709188 & -2.717039 \\
\hline 154 & 1 & 10011000 & -5.131513 & 4.946494 & 2.164539 \\
\hline 155 & 1 & 10011000 & -5.722647 & 4.934347 & -0.242909 \\
\hline 156 & 1 & 10011000 & -4.692376 & 3.303346 & -1.764674 \\
\hline 157 & 1 & 10011000 & -2.483755 & 1.628653 & 1.531169 \\
\hline 158 & 1 & 10011000 & -3.513502 & 3.291979 & 3.050600 \\
\hline 159 & 6 & 10061000 & -3.824801 & -3.042065 & 2.111416 \\
\hline 160 & 6 & 10061002 & -4.755145 & -3.567914 & 0.039424 \\
\hline 161 & 6 & 10061000 & -4.909416 & -3.336074 & -1.401292 \\
\hline 162 & 8 & 10081002 & -4.438218 & -2.377450 & -2.069645 \\
\hline 163 & 6 & 10061000 & -3.243571 & -2.208722 & 3.166134 \\
\hline 164 & 6 & 10061000 & -3.376020 & -0.794102 & 3.212116 \\
\hline 165 & 6 & 10061000 & -2.622116 & -2.844191 & 4.268690 \\
\hline 166 & 6 & 10061000 & -2.883104 & -0.055676 & 4.288038 \\
\hline 167 & 1 & 10011000 & -3.928172 & -0.282233 & 2.432428 \\
\hline 168 & 6 & 10061000 & -2.118561 & -2.115090 & 5.357317 \\
\hline 169 & 1 & 10011000 & -2.533414 & -3.929047 & 4.276120 \\
\hline 170 & 6 & 10061000 & -2.252409 & -0.710126 & 5.367671 \\
\hline 171 & 1 & 10011000 & -3.027745 & 1.018534 & 4.347244 \\
\hline 172 & 1 & 10011000 & -1.649082 & -2.640902 & 6.182448 \\
\hline 173 & 7 & 10071000 & -3.907351 & -2.753396 & 0.798029 \\
\hline 174 & 8 & 10081003 & -1.786668 & 0.122676 & 6.384070 \\
\hline 175 & 8 & 10081000 & -5.718184 & -4.295075 & -1.963881 \\
\hline 176 & 6 & 10061003 & -1.179679 & -0.478158 & 7.565585 \\
\hline 177 & 1 & 10011000 & -0.922252 & 0.360907 & 8.214525 \\
\hline 178 & 1 & 10011000 & -1.887175 & -1.140475 & 8.081203 \\
\hline 179 & 1 & 10011000 & -0.270248 & -1.037775 & 7.304396 \\
\hline 180 & 6 & 10061003 & -6.249134 & -4.005996 & -3.311265 \\
\hline 181 & 1 & 10011000 & -6.888577 & -3.120467 & -3.251057 \\
\hline 182 & 1 & 10011000 & -5.430849 & -3.833344 & -4.016547 \\
\hline 183 & 1 & 10011000 & -6.822383 & -4.893563 & -3.581704 \\
\hline 184 & 1 & 10011000 & -4.039723 & -4.073596 & 2.394150 \\
\hline 185 & 1 & 10011000 & -4.833275 & -4.611097 & 0.342456 \\
\hline 186 & 6 & 10061000 & -6.646796 & -3.174664 & 0.862722 \\
\hline 187 & 6 & 10061000 & -6.453329 & -2.953514 & 2.246867 \\
\hline 188 & 1 & 10011000 & -7.096175 & -4.055839 & 0.418518 \\
\hline 189 & 1 & 10011000 & -6.478493 & -3.684997 & 3.041376 \\
\hline 190 & 7 & 10071003 & -6.770644 & -0.878559 & 1.240595 \\
\hline 191 & 6 & 10061003 & -7.009924 & 0.552315 & 1.088404 \\
\hline 192 & 1 & 10011000 & -7.353791 & 0.951847 & 2.047084 \\
\hline 193 & 1 & 10011000 & -6.095878 & 1.089183 & 0.796515 \\
\hline 194 & 1 & 10011000 & -7.768383 & 0.712184 & 0.315707 \\
\hline 195 & 6 & 10061002 & -6.533422 & -1.521937 & 2.515549 \\
\hline 196 & 8 & 10081002 & -6.444869 & -0.870520 & 3.583876 \\
\hline 197 & 6 & 10061002 & -6.955474 & -1.833558 & 0.237262 \\
\hline 198 & 8 & 10081002 & -7.303835 & -1.587901 & -0.942453 \\
\hline
\end{tabular}

\section{TS2}

\begin{tabular}{|c|c|c|c|c|c|}
\hline \multirow{2}{*}{$\begin{array}{l}\text { Center } \\
\text { Number }\end{array}$} & \multirow{2}{*}{$\begin{array}{l}\text { Atomic } \\
\text { Number }\end{array}$} & \multirow{2}{*}{$\begin{array}{c}\text { Atomic } \\
\text { Type }\end{array}$} & \multicolumn{3}{|c|}{ Coordinates (Angstroms) } \\
\hline & & & $\mathrm{x}$ & $\mathrm{Y}$ & $\mathrm{Z}$ \\
\hline 1 & 6 & 10061000 & -2.554313 & -2.072210 & -0.000034 \\
\hline 2 & 6 & 10061002 & -1.107056 & -1.999796 & 0.316594 \\
\hline 3 & 6 & 10061002 & -0.217885 & -1.557456 & -0.581342 \\
\hline 4 & 6 & 10061000 & -0.647826 & -1.175835 & -1.936488 \\
\hline 5 & 6 & 10061002 & 0.341660 & -0.861947 & -2.985559 \\
\hline 6 & 6 & 10061000 & -0.065799 & -0.549534 & -4.226652 \\
\hline 7 & 6 & 10061000 & -1.428938 & -0.484016 & -4.552465 \\
\hline 8 & 6 & 10061002 & -2.383011 & -0.745062 & -3.641209 \\
\hline 9 & 6 & 10061000 & -2.006245 & -1.150965 & -2.261055 \\
\hline 10 & 6 & 10061000 & -3.003895 & -1.576835 & -1.246234 \\
\hline 11 & 6 & 10061002 & -4.451566 & -1.510567 & -1.566723 \\
\hline 12 & 6 & 10061000 & -5.255066 & -0.552524 & -1.024998 \\
\hline 13 & 6 & 10061000 & -6.643807 & -0.549494 & -1.277199 \\
\hline 14 & 6 & 10061002 & -7.235714 & -1.477870 & -2.043712 \\
\hline 15 & 6 & 10061000 & -6.426144 & -2.526140 & -2.684821 \\
\hline 16 & 6 & 10061000 & -5.046902 & -2.536454 & -2.475057 \\
\hline 17 & 6 & 10061002 & -4.246085 & -3.572218 & -3.179178 \\
\hline 18 & 6 & 10061000 & -4.854917 & -4.476961 & -3.967070 \\
\hline 19 & 6 & 10061000 & -6.245864 & -4.470847 & -4.153893 \\
\hline 20 & 6 & 10061002 & -7.029533 & -3.554000 & -3.559886 \\
\hline 21 & 6 & 10061000 & -4.321176 & -6.058973 & -0.918536 \\
\hline 22 & 6 & 10061000 & -5.667322 & -6.346467 & -0.682449 \\
\hline 23 & 6 & 10061000 & -6.424285 & -5.511753 & 0.142748 \\
\hline 24 & 6 & 10061000 & -5.833892 & -4.393347 & 0.738122 \\
\hline
\end{tabular}




\begin{tabular}{|c|c|c|c|c|c|}
\hline 25 & 6 & 10061000 & -4.482427 & -4.099035 & 0.511688 \\
\hline 26 & 6 & 10061000 & -3.730987 & -4.934053 & -0.334392 \\
\hline 27 & 15 & 10151003 & -3.785415 & -2.583782 & 1.292031 \\
\hline 28 & 6 & 10061000 & -2.765966 & -3.371185 & 2.610495 \\
\hline 29 & 6 & 10061000 & -1.835039 & -4.396754 & 2.363495 \\
\hline 30 & 6 & 10061000 & -1.111853 & -4.961528 & 3.418508 \\
\hline 31 & 6 & 10061000 & -1.321157 & -4.521144 & 4.727165 \\
\hline 32 & 6 & 10061000 & -2.256636 & -3.516177 & 4.983110 \\
\hline 33 & 6 & 10061000 & -2.976541 & -2.945497 & 3.929388 \\
\hline 34 & 15 & 10151003 & -4.581055 & 0.809833 & 0.012173 \\
\hline 35 & 6 & 10061000 & -5.285757 & 2.288242 & -0.810527 \\
\hline 36 & 6 & 10061000 & -4.777364 & 2.650211 & -2.064554 \\
\hline 37 & 6 & 10061000 & -5.245220 & 3.794564 & -2.715477 \\
\hline 38 & 6 & 10061000 & -6.240255 & 4.576361 & -2.124097 \\
\hline 39 & 6 & 10061000 & -6.773651 & 4.207706 & -0.886221 \\
\hline 40 & 6 & 10061000 & -6.302816 & 3.065585 & -0.230379 \\
\hline 41 & 6 & 10061000 & -5.355260 & 0.727327 & 1.670417 \\
\hline 42 & 6 & 10061000 & -6.200309 & -0.332999 & 2.039488 \\
\hline 43 & 6 & 10061000 & -6.787438 & -0.358819 & 3.307670 \\
\hline 44 & 6 & 10061000 & -6.550078 & 0.679854 & 4.210419 \\
\hline 45 & 6 & 10061000 & -5.718440 & 1.741300 & 3.847947 \\
\hline 46 & 6 & 10061000 & -5.108893 & 1.756281 & 2.590078 \\
\hline 47 & 1 & 10011000 & -4.019153 & 2.041534 & -2.536362 \\
\hline 48 & 1 & 10011000 & -4.837168 & 4.074008 & -3.678125 \\
\hline 49 & 1 & 10011000 & -6.602179 & 5.463503 & -2.627252 \\
\hline 50 & 1 & 10011000 & -7.552334 & 4.807949 & -0.433831 \\
\hline 51 & 1 & 10011000 & -6.744808 & 2.795958 & 0.716713 \\
\hline 52 & 1 & 10011000 & -4.467490 & 2.584338 & 2.322272 \\
\hline 53 & 1 & 10011000 & -5.542768 & 2.551795 & 4.543150 \\
\hline 54 & 1 & 10011000 & -7.012922 & 0.664040 & 5.188548 \\
\hline 55 & 1 & 10011000 & -7.432458 & -1.181596 & 3.587689 \\
\hline 56 & 1 & 10011000 & -6.407940 & -1.144759 & 1.359175 \\
\hline 57 & 1 & 10011000 & -1.634648 & -4.741730 & 1.363807 \\
\hline 58 & 1 & 10011000 & -0.386614 & -5.740005 & 3.220484 \\
\hline 59 & 1 & 10011000 & -0.759425 & -4.958903 & 5.542095 \\
\hline 60 & 1 & 10011000 & -2.421541 & -3.175843 & 5.997127 \\
\hline 61 & 1 & 10011000 & -3.700826 & -2.168627 & 4.141382 \\
\hline 62 & 1 & 10011000 & -7.265097 & 0.218068 & -0.832753 \\
\hline 63 & 1 & 10011000 & -8.309123 & -1.444175 & -2.203594 \\
\hline 64 & 1 & 10011000 & -8.102527 & -3.569065 & -3.724997 \\
\hline 65 & 1 & 10011000 & -6.695903 & -5.219850 & -4.792954 \\
\hline 66 & 1 & 10011000 & -4.259622 & -5.231583 & -4.465206 \\
\hline 67 & 1 & 10011000 & -3.169534 & -3.619437 & -3.055307 \\
\hline 68 & 1 & 10011000 & -3.425516 & -0.669459 & -3.931700 \\
\hline 69 & 1 & 10011000 & -1.717564 & -0.206570 & -5.558257 \\
\hline 70 & 1 & 10011000 & 0.672073 & -0.329309 & -4.987864 \\
\hline 71 & 1 & 10011000 & 1.396996 & -0.909046 & -2.757105 \\
\hline 72 & 1 & 10011000 & 0.828706 & -1.488244 & -0.307774 \\
\hline 73 & 1 & 10011000 & -0.746609 & -2.282964 & 1.291203 \\
\hline 74 & 1 & 10011000 & -6.125747 & -7.210266 & -1.146001 \\
\hline 75 & 1 & 10011000 & -3.740222 & -6.694554 & -1.574163 \\
\hline 76 & 1 & 10011000 & -2.704741 & -4.703675 & -0.580264 \\
\hline 77 & 1 & 10011000 & -6.432495 & -3.752490 & 1.373020 \\
\hline 78 & 1 & 10011000 & -7.469336 & -5.730109 & 0.319942 \\
\hline 79 & 79 & 10791004 & 3.290268 & -0.844689 & -0.887986 \\
\hline 80 & 79 & 10791004 & -2.141136 & 1.253229 & -0.011636 \\
\hline 81 & 6 & 10061000 & 1.534335 & 0.544742 & 1.982534 \\
\hline 82 & 6 & 10061002 & 1.010627 & -0.693186 & 2.613354 \\
\hline 83 & 6 & 10061002 & -0.075211 & -0.682243 & 3.393486 \\
\hline 84 & 6 & 10061000 & -0.827003 & 0.561677 & 3.607755 \\
\hline 85 & 6 & 10061002 & -1.934915 & 0.613260 & 4.585828 \\
\hline 86 & 6 & 10061000 & -2.549326 & 1.778444 & 4.843734 \\
\hline 87 & 6 & 10061000 & -2.192272 & 2.957687 & 4.175259 \\
\hline 88 & 6 & 10061002 & -1.205692 & 2.977267 & 3.262459 \\
\hline 89 & 6 & 10061000 & -0.446879 & 1.735600 & 2.947112 \\
\hline 90 & 6 & 10061000 & 0.769043 & 1.739410 & 2.081020 \\
\hline 91 & 6 & 10061002 & 1.285634 & 3.052634 & 1.638318 \\
\hline 92 & 6 & 10061002 & 0.884095 & 3.650415 & 0.481926 \\
\hline 93 & 6 & 10061002 & 1.446611 & 4.991170 & 0.139043 \\
\hline 94 & 6 & 10061000 & 2.243040 & 5.656971 & 0.985938 \\
\hline 95 & 6 & 10061000 & 2.562482 & 5.125316 & 2.235039 \\
\hline 96 & 6 & 10061000 & 2.096524 & 3.855236 & 2.606233 \\
\hline 97 & 6 & 10061002 & 2.402835 & 3.380593 & 3.983533 \\
\hline 98 & 6 & 10061000 & 3.144229 & 4.136016 & 4.812189 \\
\hline 99 & 6 & 10061000 & 3.638540 & 5.387884 & 4.419607 \\
\hline 100 & 6 & 10061002 & 3.381816 & 5.893600 & 3.201256 \\
\hline 101 & 6 & 10061000 & 5.630639 & 3.610641 & 1.910848 \\
\hline 102 & 6 & 10061000 & 5.713258 & 4.201245 & 0.647535 \\
\hline 103 & 6 & 10061000 & 5.030246 & 3.635908 & -0.431477 \\
\hline 104 & 6 & 10061000 & 4.253986 & 2.489852 & -0.242491 \\
\hline
\end{tabular}




\begin{tabular}{|c|c|c|c|c|c|}
\hline 105 & 6 & 10061000 & 4.120403 & 1.922545 & 1.029808 \\
\hline 106 & 6 & 10061000 & 4.845680 & 2.468992 & 2.101722 \\
\hline 107 & 15 & 10151003 & 3.189726 & 0.355719 & 1.171531 \\
\hline 108 & 6 & 10061000 & 4.155536 & -0.630546 & 2.373891 \\
\hline 109 & 6 & 10061000 & 4.974585 & -1.663045 & 1.902569 \\
\hline 110 & 6 & 10061000 & 5.700703 & -2.452453 & 2.798981 \\
\hline 111 & 6 & 10061000 & 5.595724 & -2.226785 & 4.173729 \\
\hline 112 & 6 & 10061000 & 4.767580 & -1.207965 & 4.652593 \\
\hline 113 & 6 & 10061000 & 4.047122 & -0.412335 & 3.756898 \\
\hline 114 & 15 & 10151003 & -0.469353 & 2.998278 & -0.599783 \\
\hline 115 & 6 & 10061000 & -1.609397 & 4.434873 & -0.646813 \\
\hline 116 & 6 & 10061000 & -2.672547 & 4.470231 & 0.264825 \\
\hline 117 & 6 & 10061000 & -3.593094 & 5.520765 & 0.239662 \\
\hline 118 & 6 & 10061000 & -3.461553 & 6.542295 & -0.703656 \\
\hline 119 & 6 & 10061000 & -2.406768 & 6.514571 & -1.619785 \\
\hline 120 & 6 & 10061000 & -1.482610 & 5.465306 & -1.594790 \\
\hline 121 & 6 & 10061000 & 0.122104 & 2.911841 & -2.331431 \\
\hline 122 & 6 & 10061000 & -0.805706 & 2.660391 & -3.351090 \\
\hline 123 & 6 & 10061000 & -0.416594 & 2.715442 & -4.692458 \\
\hline 124 & 6 & 10061000 & 0.907408 & 3.008788 & -5.023958 \\
\hline 125 & 6 & 10061000 & 1.840274 & 3.252668 & -4.013528 \\
\hline 126 & 6 & 10061000 & 1.451186 & 3.200348 & -2.672573 \\
\hline 127 & 1 & 10011000 & -2.796947 & 3.675263 & 0.985693 \\
\hline 128 & 1 & 10011000 & -4.414615 & 5.536156 & 0.944154 \\
\hline 129 & 1 & 10011000 & -4.179087 & 7.352075 & -0.727840 \\
\hline 130 & 1 & 10011000 & -2.307223 & 7.304797 & -2.352540 \\
\hline 131 & 1 & 10011000 & -0.678467 & 5.462989 & -2.317241 \\
\hline 132 & 1 & 10011000 & 2.181197 & 3.421400 & -1.914469 \\
\hline 133 & 1 & 10011000 & 2.863180 & 3.496270 & -4.267925 \\
\hline 134 & 1 & 10011000 & 1.208042 & 3.054048 & -6.062482 \\
\hline 135 & 1 & 10011000 & -1.142127 & 2.531154 & -5.474006 \\
\hline 136 & 1 & 10011000 & -1.835066 & 2.447047 & -3.107558 \\
\hline 137 & 1 & 10011000 & 5.052037 & -1.853963 & 0.841388 \\
\hline 138 & 1 & 10011000 & 6.340032 & -3.242891 & 2.428392 \\
\hline 139 & 1 & 10011000 & 6.152829 & -2.843038 & 4.867353 \\
\hline 140 & 1 & 10011000 & 4.681090 & -1.036982 & 5.717738 \\
\hline 141 & 1 & 10011000 & 3.400144 & 0.363712 & 4.142401 \\
\hline 142 & 1 & 10011000 & 1.204779 & 5.452231 & -0.811044 \\
\hline 143 & 1 & 10011000 & 2.619719 & 6.631773 & 0.700670 \\
\hline 144 & 1 & 10011000 & 3.775776 & 6.868921 & 2.930814 \\
\hline 145 & 1 & 10011000 & 4.236648 & 5.960938 & 5.116617 \\
\hline 146 & 1 & 10011000 & 3.366561 & 3.769919 & 5.806466 \\
\hline 147 & 1 & 10011000 & 2.039691 & 2.420418 & 4.332641 \\
\hline 148 & 1 & 10011000 & -0.960037 & 3.915856 & 2.777107 \\
\hline 149 & 1 & 10011000 & -2.724178 & 3.873143 & 4.401279 \\
\hline 150 & 1 & 10011000 & -3.340663 & 1.807433 & 5.581399 \\
\hline 151 & 1 & 10011000 & -2.231987 & -0.282027 & 5.122494 \\
\hline 152 & 1 & 10011000 & -0.395672 & -1.600152 & 3.873026 \\
\hline 153 & 1 & 10011000 & 1.537538 & -1.632563 & 2.472906 \\
\hline 154 & 1 & 10011000 & 6.317028 & 5.087434 & 0.502313 \\
\hline 155 & 1 & 10011000 & 6.171634 & 4.039613 & 2.744244 \\
\hline 156 & 1 & 10011000 & 4.804583 & 2.030824 & 3.085422 \\
\hline 157 & 1 & 10011000 & 3.774801 & 2.025531 & -1.091940 \\
\hline 158 & 1 & 10011000 & 5.114266 & 4.075222 & -1.416954 \\
\hline 159 & 6 & 10061000 & 4.155081 & -3.427899 & -2.107764 \\
\hline 160 & 6 & 10061002 & 4.330764 & -1.663897 & -3.640560 \\
\hline 161 & 6 & 10061000 & 3.967695 & -0.299982 & -4.039948 \\
\hline 162 & 8 & 10081002 & 3.389254 & 0.570970 & -3.340179 \\
\hline 163 & 6 & 10061000 & 3.744120 & -4.037257 & -0.825500 \\
\hline 164 & 6 & 10061000 & 2.389385 & -3.970245 & -0.406812 \\
\hline 165 & 6 & 10061000 & 4.677075 & -4.678857 & 0.017445 \\
\hline 166 & 6 & 10061000 & 1.991215 & -4.463930 & 0.839162 \\
\hline 167 & 1 & 10011000 & 1.649993 & -3.567160 & -1.092893 \\
\hline 168 & 6 & 10061000 & 4.285574 & -5.183143 & 1.271479 \\
\hline 169 & 1 & 10011000 & 5.724758 & -4.722545 & -0.266010 \\
\hline 170 & 6 & 10061000 & 2.946993 & -5.051521 & 1.696352 \\
\hline 171 & 1 & 10011000 & 0.955951 & -4.425269 & 1.162203 \\
\hline 172 & 1 & 10011000 & 5.034228 & -5.642854 & 1.908209 \\
\hline 173 & 7 & 10071000 & 3.836582 & -2.171355 & -2.434133 \\
\hline 174 & 8 & 10081003 & 2.463344 & -5.467938 & 2.935864 \\
\hline 175 & 8 & 10081000 & 4.421191 & -0.054174 & -5.318453 \\
\hline 176 & 6 & 10061003 & 3.387736 & -6.099322 & 3.872439 \\
\hline 177 & 1 & 10011000 & 2.788674 & -6.332311 & 4.754572 \\
\hline 178 & 1 & 10011000 & 3.805458 & -7.024180 & 3.453553 \\
\hline 179 & 1 & 10011000 & 4.202839 & -5.415165 & 4.145543 \\
\hline 180 & 6 & 10061003 & 4.420864 & 1.340137 & -5.788462 \\
\hline 181 & 1 & 10011000 & 3.400187 & 1.655112 & -6.026189 \\
\hline 182 & 1 & 10011000 & 4.854289 & 1.985786 & -5.019829 \\
\hline 183 & 1 & 10011000 & 5.037439 & 1.332605 & -6.688457 \\
\hline 184 & 6 & 10061000 & 6.378487 & -1.624657 & -3.379952 \\
\hline
\end{tabular}




\begin{tabular}{|c|c|c|c|c|c|}
\hline 185 & 6 & 10061000 & 6.775143 & -2.763744 & -2.636365 \\
\hline 186 & 1 & 10011000 & 4.607548 & -4.061426 & -2.867080 \\
\hline 187 & 1 & 10011000 & 4.400557 & -2.365708 & -4.470356 \\
\hline 188 & 1 & 10011000 & 6.996772 & -3.750191 & -3.017056 \\
\hline 189 & 1 & 10011000 & 6.558867 & -1.479715 & -4.440279 \\
\hline 190 & 6 & 10061002 & 7.111945 & -2.366923 & -1.281875 \\
\hline 191 & 8 & 10081002 & 7.497836 & -3.035704 & -0.288849 \\
\hline 192 & 6 & 10061002 & 6.588184 & -0.423428 & -2.473248 \\
\hline 193 & 8 & 10081002 & 6.468868 & 0.792743 & -2.757445 \\
\hline 194 & 7 & 10071003 & 6.949711 & -0.925265 & -1.224335 \\
\hline 195 & 6 & 10061003 & 7.378144 & -0.111589 & -0.092311 \\
\hline 196 & 1 & 10011000 & 8.202518 & 0.551441 & -0.380038 \\
\hline 197 & 1 & 10011000 & 6.559941 & 0.514676 & 0.283714 \\
\hline 198 & 1 & 10011000 & 7.714570 & -0.793507 & 0.694434 \\
\hline
\end{tabular}

\section{TS3}

\begin{tabular}{|c|c|c|c|c|c|}
\hline \multirow{2}{*}{$\begin{array}{l}\text { Center } \\
\text { Number }\end{array}$} & \multirow{2}{*}{$\begin{array}{l}\text { Atomic } \\
\text { Number }\end{array}$} & \multirow{2}{*}{$\begin{array}{c}\text { Atomic } \\
\text { Type }\end{array}$} & \multicolumn{3}{|c|}{ Coordinates (Angstroms) } \\
\hline & & & $\mathrm{x}$ & $\mathrm{Y}$ & $\mathrm{Z}$ \\
\hline & & & & & \\
\hline 1 & 6 & 10061000 & -2.041199 & 0.924479 & 0.928679 \\
\hline 2 & 6 & 10061000 & -1.204944 & 0.038974 & 1.766504 \\
\hline 3 & 6 & 10061000 & -0.763140 & 0.425695 & 3.026882 \\
\hline 4 & 6 & 10061000 & -1.062626 & 1.686235 & 3.537534 \\
\hline 5 & 6 & 10061000 & -0.658018 & 2.056079 & 4.825345 \\
\hline 6 & 6 & 10061000 & -1.010975 & 3.298283 & 5.361785 \\
\hline 7 & 6 & 10061000 & -1.799489 & 4.206946 & 4.649940 \\
\hline 8 & 6 & 10061000 & -2.245209 & 3.916533 & 3.362161 \\
\hline 9 & 6 & 10061002 & -1.850948 & 2.641170 & 2.723137 \\
\hline 10 & 6 & 10061000 & -2.293936 & 2.249153 & 1.356782 \\
\hline 11 & 6 & 10061000 & -3.038544 & 3.223946 & 0.527904 \\
\hline 12 & 6 & 10061000 & -2.385028 & 4.061008 & -0.403272 \\
\hline 13 & 6 & 10061000 & -3.207981 & 4.903956 & -1.308021 \\
\hline 14 & 6 & 10061000 & -4.594316 & 4.969723 & -1.193859 \\
\hline 15 & 6 & 10061000 & -5.264908 & 4.269547 & -0.193460 \\
\hline 16 & 6 & 10061002 & -4.499642 & 3.409141 & 0.737847 \\
\hline 17 & 6 & 10061000 & -5.229221 & 2.838683 & 1.891254 \\
\hline 18 & 6 & 10061000 & -6.608550 & 3.013796 & 1.985194 \\
\hline 19 & 6 & 10061000 & -7.307613 & 3.755050 & 1.027629 \\
\hline 20 & 6 & 10061000 & -6.651122 & 4.383889 & -0.035771 \\
\hline 21 & 6 & 10061000 & -0.093277 & 7.579480 & -2.826562 \\
\hline 22 & 6 & 10061000 & 0.593831 & 7.072331 & -3.932341 \\
\hline 23 & 6 & 10061000 & 0.927265 & 5.717146 & -3.984186 \\
\hline 24 & 6 & 10061000 & 0.576076 & 4.870131 & -2.929734 \\
\hline 25 & 6 & 10061000 & -0.118455 & 5.367190 & -1.819667 \\
\hline 26 & 6 & 10061000 & -0.448024 & 6.732473 & -1.771830 \\
\hline 27 & 15 & 10151003 & -0.546799 & 4.205769 & -0.469352 \\
\hline 28 & 6 & 10061000 & -0.049653 & 5.084140 & 1.050770 \\
\hline 29 & 6 & 10061000 & -0.813170 & 6.139744 & 1.572564 \\
\hline 30 & 6 & 10061000 & -0.407526 & 6.786699 & 2.744275 \\
\hline 31 & 6 & 10061000 & 0.752136 & 6.373873 & 3.406879 \\
\hline 32 & 6 & 10061000 & 1.508371 & 5.315024 & 2.896825 \\
\hline 33 & 6 & 10061000 & 1.109127 & 4.674538 & 1.720603 \\
\hline 34 & 15 & 10151003 & -2.829867 & 0.112405 & -0.521238 \\
\hline 35 & 6 & 10061000 & -4.623496 & 0.011713 & -0.178124 \\
\hline 36 & 6 & 10061000 & -5.543718 & -0.143952 & -1.226493 \\
\hline 37 & 6 & 10061000 & -6.900873 & -0.337522 & -0.952551 \\
\hline 38 & 6 & 10061000 & -7.340808 & -0.434845 & 0.369416 \\
\hline 39 & 6 & 10061000 & -6.420300 & -0.359173 & 1.416734 \\
\hline 40 & 6 & 10061000 & -5.064920 & -0.153758 & 1.143516 \\
\hline 41 & 6 & 10061000 & -2.489089 & 1.150853 & -1.986705 \\
\hline 42 & 6 & 10061000 & -1.219978 & 1.059403 & -2.569479 \\
\hline 43 & 6 & 10061000 & -0.921175 & 1.772037 & -3.733280 \\
\hline 44 & 6 & 10061000 & -1.889756 & 2.590978 & -4.318168 \\
\hline 45 & 6 & 10061000 & -3.159328 & 2.688897 & -3.743007 \\
\hline 46 & 6 & 10061000 & -3.461056 & 1.970769 & -2.580920 \\
\hline 47 & 1 & 10011000 & -5.213526 & -0.136365 & -2.256126 \\
\hline 48 & 1 & 10011000 & -7.607733 & -0.435982 & -1.766097 \\
\hline 49 & 1 & 10011000 & -8.390150 & -0.593441 & 0.581159 \\
\hline 50 & 1 & 10011000 & -6.755085 & -0.465112 & 2.440452 \\
\hline 51 & 1 & 10011000 & -4.362195 & -0.130048 & 1.965550 \\
\hline 52 & 1 & 10011000 & -4.436602 & 2.091028 & -2.135153 \\
\hline 53 & 1 & 10011000 & -3.906799 & 3.331422 & -4.189887 \\
\hline 54 & 1 & 10011000 & -1.655689 & 3.151622 & -5.213736 \\
\hline 55 & 1 & 10011000 & 0.062040 & 1.692101 & -4.178876 \\
\hline 56 & 1 & 10011000 & -0.467596 & 0.424142 & -2.123238 \\
\hline
\end{tabular}




\begin{tabular}{|c|c|c|c|c|c|}
\hline 57 & 1 & 10011000 & -1.736605 & 6.441225 & 1.097157 \\
\hline 58 & 1 & 10011000 & -1.001796 & 7.595900 & 3.148173 \\
\hline 59 & 1 & 10011000 & 1.059801 & 6.868794 & 4.318749 \\
\hline 60 & 1 & 10011000 & 2.403861 & 4.991126 & 3.411331 \\
\hline 61 & 1 & 10011000 & 1.702933 & 3.862629 & 1.328415 \\
\hline 62 & 1 & 10011000 & -2.748509 & 5.455832 & -2.112409 \\
\hline 63 & 1 & 10011000 & -5.146072 & 5.592546 & -1.888000 \\
\hline 64 & 1 & 10011000 & -7.231958 & 4.983719 & -0.726353 \\
\hline 65 & 1 & 10011000 & -8.379520 & 3.867610 & 1.127191 \\
\hline 66 & 1 & 10011000 & -7.146493 & 2.568634 & 2.812178 \\
\hline 67 & 1 & 10011000 & -4.712701 & 2.274607 & 2.655625 \\
\hline 68 & 1 & 10011000 & -2.870547 & 4.630866 & 2.844319 \\
\hline 69 & 1 & 10011000 & -2.070943 & 5.149729 & 5.107018 \\
\hline 70 & 1 & 10011000 & -0.682966 & 3.554097 & 6.361023 \\
\hline 71 & 1 & 10011000 & -0.079587 & 1.372139 & 5.433320 \\
\hline 72 & 1 & 10011000 & -0.213331 & -0.286482 & 3.625370 \\
\hline 73 & 1 & 10011000 & -0.968311 & -0.958837 & 1.419177 \\
\hline 74 & 1 & 10011000 & 0.866168 & 7.728529 & -4.748767 \\
\hline 75 & 1 & 10011000 & -0.355117 & 8.628954 & -2.788333 \\
\hline 76 & 1 & 10011000 & -0.993632 & 7.145121 & -0.939195 \\
\hline 77 & 1 & 10011000 & 0.843644 & 3.824427 & -2.980123 \\
\hline 78 & 1 & 10011000 & 1.458255 & 5.322561 & -4.840744 \\
\hline 79 & 79 & 10791004 & 0.934367 & 2.266608 & -0.768568 \\
\hline 80 & 79 & 10791004 & -2.037035 & -2.095706 & -0.780042 \\
\hline 81 & 6 & 10061000 & 3.014545 & -0.662853 & -1.541071 \\
\hline 82 & 6 & 10061000 & 2.090910 & -1.121269 & -2.604363 \\
\hline 83 & 6 & 10061000 & 2.028625 & -2.449787 & -3.010260 \\
\hline 84 & 6 & 10061000 & 2.832564 & -3.418747 & -2.417294 \\
\hline 85 & 6 & 10061000 & 2.765980 & -4.756716 & -2.823719 \\
\hline 86 & 6 & 10061000 & 3.591870 & -5.726140 & -2.246008 \\
\hline 87 & 6 & 10061000 & 4.516692 & -5.402583 & -1.248658 \\
\hline 88 & 6 & 10061000 & 4.641925 & -4.096110 & -0.780510 \\
\hline 89 & 6 & 10061002 & 3.781063 & -3.030713 & -1.347098 \\
\hline 90 & 6 & 10061000 & 3.827139 & -1.615094 & -0.880819 \\
\hline 91 & 6 & 10061000 & 4.732760 & -1.276789 & 0.240279 \\
\hline 92 & 6 & 10061000 & 4.269332 & -1.252028 & 1.573498 \\
\hline 93 & 6 & 10061000 & 5.256933 & -1.111398 & 2.675950 \\
\hline 94 & 6 & 10061000 & 6.620415 & -0.972184 & 2.424872 \\
\hline 95 & 6 & 10061000 & 7.119089 & -0.985636 & 1.123025 \\
\hline 96 & 6 & 10061002 & 6.191384 & -1.166442 & -0.016373 \\
\hline 97 & 6 & 10061000 & 6.767797 & -1.179768 & -1.379765 \\
\hline 98 & 6 & 10061000 & 8.141579 & -1.014341 & -1.542587 \\
\hline 99 & 6 & 10061000 & 8.983280 & -0.843309 & -0.438441 \\
\hline 100 & 6 & 10061000 & 8.487174 & -0.831104 & 0.870086 \\
\hline 101 & 6 & 10061000 & 6.584515 & 2.352955 & 0.491433 \\
\hline 102 & 6 & 10061000 & 6.182390 & 2.636239 & 1.799016 \\
\hline 103 & 6 & 10061000 & 4.848557 & 2.455864 & 2.173428 \\
\hline 104 & 6 & 10061000 & 3.918838 & 1.977732 & 1.244554 \\
\hline 105 & 6 & 10061000 & 4.318465 & 1.672589 & -0.061305 \\
\hline 106 & 6 & 10061000 & 5.654592 & 1.883345 & -0.440670 \\
\hline 107 & 15 & 10151003 & 3.056755 & 1.159012 & -1.276619 \\
\hline 108 & 6 & 10061000 & 3.674543 & 1.897337 & -2.834732 \\
\hline 109 & 6 & 10061000 & 3.664512 & 3.292964 & -2.964680 \\
\hline 110 & 6 & 10061000 & 4.143903 & 3.897792 & -4.129742 \\
\hline 111 & 6 & 10061000 & 4.647335 & 3.111896 & -5.169062 \\
\hline 112 & 6 & 10061000 & 4.681288 & 1.721015 & -5.038996 \\
\hline 113 & 6 & 10061000 & 4.203013 & 1.112937 & -3.874182 \\
\hline 114 & 15 & 10151003 & 2.447813 & -1.428490 & 1.907083 \\
\hline 115 & 6 & 10061000 & 2.412947 & -3.261720 & 1.743794 \\
\hline 116 & 6 & 10061000 & 1.492294 & -3.843555 & 0.863349 \\
\hline 117 & 6 & 10061000 & 1.487417 & -5.227713 & 0.664497 \\
\hline 118 & 6 & 10061000 & 2.383382 & -6.040099 & 1.362953 \\
\hline 119 & 6 & 10061000 & 3.301195 & -5.467886 & 2.246717 \\
\hline 120 & 6 & 10061000 & 3.325275 & -4.082761 & 2.430561 \\
\hline 121 & 6 & 10061000 & 2.346398 & -1.239247 & 3.747098 \\
\hline 122 & 6 & 10061000 & 1.803095 & -2.241357 & 4.573077 \\
\hline 123 & 6 & 10061000 & 1.657196 & -2.026209 & 5.946637 \\
\hline 124 & 6 & 10061000 & 2.024658 & -0.801867 & 6.507289 \\
\hline 125 & 6 & 10061000 & 2.525123 & 0.214350 & 5.691923 \\
\hline 126 & 6 & 10061000 & 2.664619 & 0.003543 & 4.317791 \\
\hline 127 & 1 & 10011000 & 0.798947 & -3.221593 & 0.311735 \\
\hline 128 & 1 & 10011000 & 0.794210 & -5.672933 & -0.034555 \\
\hline 129 & 1 & 10011000 & 2.374611 & -7.111268 & 1.209613 \\
\hline 130 & 1 & 10011000 & 4.001744 & -6.096481 & 2.780721 \\
\hline 131 & 1 & 10011000 & 4.048709 & -3.654377 & 3.110655 \\
\hline 132 & 1 & 10011000 & 3.024126 & 0.809427 & 3.698961 \\
\hline 133 & 1 & 10011000 & 2.788250 & 1.172345 & 6.121219 \\
\hline 134 & 1 & 10011000 & 1.905611 & -0.634954 & 7.569906 \\
\hline 135 & 1 & 10011000 & 1.243785 & -2.805044 & 6.574342 \\
\hline 136 & 1 & 10011000 & 1.465002 & -3.180813 & 4.166196 \\
\hline
\end{tabular}




\begin{tabular}{|c|c|c|c|c|c|}
\hline 137 & 1 & 10011000 & 3.288632 & 3.912439 & -2.159987 \\
\hline 138 & 1 & 10011000 & 4.127910 & 4.975769 & -4.225085 \\
\hline 139 & 1 & 10011000 & 5.019708 & 3.580729 & -6.070640 \\
\hline 140 & 1 & 10011000 & 5.085701 & 1.113728 & -5.838409 \\
\hline 141 & 1 & 10011000 & 4.268630 & 0.038365 & -3.787602 \\
\hline 142 & 1 & 10011000 & 4.947405 & -1.151533 & 3.707529 \\
\hline 143 & 1 & 10011000 & 7.295876 & -0.862302 & 3.265049 \\
\hline 144 & 1 & 10011000 & 9.182056 & -0.695133 & 1.690332 \\
\hline 145 & 1 & 10011000 & 10.045979 & -0.716922 & -0.600664 \\
\hline 146 & 1 & 10011000 & 8.563317 & -1.015332 & -2.539337 \\
\hline 147 & 1 & 10011000 & 6.137909 & -1.296758 & -2.251761 \\
\hline 148 & 1 & 10011000 & 5.355019 & -3.883703 & 0.004265 \\
\hline 149 & 1 & 10011000 & 5.137795 & -6.181260 & -0.825223 \\
\hline 150 & 1 & 10011000 & 3.514764 & -6.752467 & -2.581015 \\
\hline 151 & 1 & 10011000 & 2.070883 & -5.058499 & -3.598168 \\
\hline 152 & 1 & 10011000 & 1.357422 & -2.717213 & -3.816571 \\
\hline 153 & 1 & 10011000 & 1.481839 & -0.396727 & -3.127057 \\
\hline 154 & 1 & 10011000 & 6.902610 & 3.000107 & 2.520069 \\
\hline 155 & 1 & 10011000 & 7.616820 & 2.496039 & 0.199786 \\
\hline 156 & 1 & 10011000 & 5.983465 & 1.673170 & -1.449279 \\
\hline 157 & 1 & 10011000 & 2.887816 & 1.839276 & 1.540831 \\
\hline 158 & 1 & 10011000 & 4.535654 & 2.689237 & 3.182646 \\
\hline 159 & 7 & 10071000 & -1.682202 & -4.170832 & -0.700849 \\
\hline 160 & 6 & 10061002 & -2.099178 & -4.907495 & 0.400351 \\
\hline 161 & 6 & 10061000 & -1.380447 & -4.849935 & -1.831774 \\
\hline 162 & 1 & 10011000 & -1.723939 & -5.935806 & 0.403220 \\
\hline 163 & 6 & 10061000 & -2.098023 & -4.320063 & 1.777347 \\
\hline 164 & 6 & 10061000 & -1.242865 & -4.908710 & 2.744491 \\
\hline 165 & 6 & 10061000 & -2.883490 & -3.217165 & 2.177602 \\
\hline 166 & 6 & 10061000 & -1.107663 & -4.367410 & 4.027331 \\
\hline 167 & 1 & 10011000 & -0.667828 & -5.795437 & 2.485213 \\
\hline 168 & 6 & 10061000 & -2.759296 & -2.662769 & 3.464568 \\
\hline 169 & 1 & 10011000 & -3.635389 & -2.825467 & 1.501394 \\
\hline 170 & 6 & 10061000 & -1.845505 & -3.218756 & 4.383326 \\
\hline 171 & 1 & 10011000 & -0.444471 & -4.809198 & 4.764655 \\
\hline 172 & 1 & 10011000 & -3.390233 & -1.824451 & 3.744301 \\
\hline 173 & 8 & 10081003 & -1.604598 & -2.723777 & 5.664290 \\
\hline 174 & 6 & 10061003 & -2.359757 & -1.568597 & 6.128323 \\
\hline 175 & 1 & 10011000 & -1.986130 & -1.366610 & 7.133761 \\
\hline 176 & 1 & 10011000 & -2.185082 & -0.693092 & 5.486494 \\
\hline 177 & 1 & 10011000 & -3.435199 & -1.786890 & 6.169820 \\
\hline 178 & 6 & 10061000 & -1.172142 & -4.117222 & -3.083224 \\
\hline 179 & 8 & 10081002 & -1.250680 & -2.859683 & -3.173120 \\
\hline 180 & 8 & 10081000 & -0.841862 & -4.812293 & -4.221078 \\
\hline 181 & 6 & 10061003 & -0.940269 & -6.282029 & -4.307919 \\
\hline 182 & 1 & 10011000 & -1.962859 & -6.603529 & -4.088817 \\
\hline 183 & 1 & 10011000 & -0.693687 & -6.506060 & -5.346607 \\
\hline 184 & 1 & 10011000 & -0.209491 & -6.762162 & -3.644559 \\
\hline 185 & 1 & 10011000 & -1.113855 & -5.897809 & -1.767830 \\
\hline 186 & 6 & 10061000 & -3.716610 & -6.262525 & -1.433607 \\
\hline 187 & 6 & 10061000 & -3.937241 & -5.506675 & -0.255722 \\
\hline 188 & 1 & 10011000 & -3.374403 & -7.286299 & -1.496373 \\
\hline 189 & 1 & 10011000 & -4.199352 & -5.937580 & 0.707354 \\
\hline 190 & 6 & 10061002 & -4.232412 & -5.548266 & -2.592662 \\
\hline 191 & 8 & 10081002 & -4.274068 & -5.865554 & -3.806153 \\
\hline 192 & 6 & 10061002 & -4.698601 & -4.259660 & -0.701501 \\
\hline 193 & 8 & 10081002 & -5.157156 & -3.325393 & 0.006305 \\
\hline 194 & 7 & 10071000 & -4.794380 & -4.318062 & -2.085059 \\
\hline 195 & 6 & 10061003 & -5.439747 & -3.320514 & -2.934419 \\
\hline 196 & 1 & 10011000 & -4.763730 & -2.482850 & -3.146742 \\
\hline 197 & 1 & 10011000 & -5.701657 & -3.802987 & -3.880126 \\
\hline 198 & 1 & 10011000 & -6.341821 & -2.944772 & -2.441730 \\
\hline
\end{tabular}

Complete reference 16:

Gaussian 09, Revision A.1, M. J. Frisch, G. W. Trucks, H. B. Schlegel, G. E. Scuseria, M. A. Robb, J. R. Cheeseman, G. Scalmani, V. Barone, B. Mennucci, G. A. Petersson, H. Nakatsuji, M. Caricato, X. Li, H. P. Hratchian, A. F. Izmaylov, J. Bloino, G. Zheng, J. L. Sonnenberg, M. Hada, M. Ehara, K. Toyota, R. Fukuda, J. Hasegawa, M. Ishida, T. Nakajima, Y. Honda, O. Kitao, H. Nakai, T. Vreven, J. A. Montgomery, Jr., J. E. Peralta, F. Ogliaro, M. Bearpark, J. J. Heyd, E. Brothers, K. N. Kudin, V. N. Staroverov, R. Kobayashi, J. Normand, K. Raghavachari, A. Rendell, J. C. Burant, S. S. Iyengar, J. Tomasi, M. Cossi, N. Rega, J. M. Millam, M. Klene, J. E. Knox, J. B. Cross, V. Bakken, C. Adamo, J. Jaramillo, R. Gomperts, R. E. Stratmann, O. Yazyev, A. J. Austin, R. Cammi, C. Pomelli, J. W. Ochterski, R. L. Martin, K. Morokuma, V. G. Zakrzewski, G. A. Voth, P. Salvador, J. J. Dannenberg, S. 
Dapprich, A. D. Daniels, Ö. Farkas, J. B. Foresman, J. V. Ortiz, J. Cioslowski, and D. J. Fox, Gaussian, Inc., Wallingford CT, 2009. 